\title{
578.
}

\section{A MEMOIR ON THE TRANSFORMATION OF ELLIPTIC FUNCTIONS.}

[From the Philosophical Transactions of the Royal Society of London, vol. CLXIV. (for the year 1874), pp. 397-456. Received November 14, 1873,-Read January 8, 1874.]

THE theory of Transformation in Elliptic Functions was established by Jacobi in the Fundamenta Nova (1829); and he has there developed, transcendentally, with an approach to completeness, the general case, $n$ an odd number, but algebraically only the cases $n=3$ and $n=5$; viz. in the general case the formulæ are expressed in terms of the elliptic functions of the $n$th part of the complete integrals, but in the cases $n=3$ and $n=5$ they are expressed rationally in terms of $u$ and $v$ (the fourth roots of the original and the transformed moduli respectively), these quantities being connected by an equation of the order 4 or 6 , the modular equation. The extension of this algebraical theory to any value whatever of $n$ is a problem of great interest and difficulty: such theory should admit of being treated in a purely algebraical manner; but the difficulties are so great that it was found necessary to discuss it by means of the formulæ of the transcendental theory, in particular by means of the expressions involving Jacobi's $q$ (the exponential of $-\frac{\pi K^{\prime}}{K}$ ), or say by means of the $q$-transcendents. Several important contributions to the theory have since been made:-Sohnke, "Equationes modulares pro transformatione functionum ellipticarum," Crelle, t. xvi. (1836), pp. 97-130, (where the modular equations are found for the cases $n=3,5,7,11,13$, 17, and 19); Joubert, "Sur divers équations analogues aux équations modulaires dans la théorie des fonctions elliptiques," Comptes Rendus, t. XLVII. (1858), pp. 337-345, (relating among other things to the multiplier equation for the determination of Jacobi's $M)$; and Königsberger, "Algebraische Untersuchungen aus der Theorie der elliptischen Functionen," Crelle, t. LxxII. (1870), pp. 176-275; together with other papers by Joubert and by Hermite in later volumes of the Comptes Rendus, which need not be more particularly referred to. In the present Memoir I carry on the theory, algebraically, as far as I am able; and I have, it appears to me, put the purely

c. IX. 
algebraical question in a clearer light than has hitherto been done; but I still find it necessary to resort to the transcendental theory. I remark that the case $n=7$ (next succeeding those of the Fundamenta Nova), on account of the peculiarly simple form of the modular equation $\left(1-u^{8}\right)\left(1-v^{8}\right)=(1-u v)^{8}$, presents but little difficulty ; and I give the complete formulæ for this case, obtaining them as well algebraically as transcendentally; I also to a considerable extent discuss algebraically the case of the next succeeding prime value $n=11$. For the sake of completeness I reproduce Sohnke's modular equations, exhibiting them for greater clearness in a square form, and adding to them those for the non-prime cases $n=9$ and $n=15$; also a valuable table given by him for the powers of $f(q)$; and I give other tabular results which are of assistance in the theory.

The General Problem. Article Nos. 1 to 6.

1. Taking $n$ a given odd number, I write

$$
\frac{1-y}{1+y}=\frac{1-x}{1+x}\left(\frac{P-Q x}{P+Q x}\right)^{2}
$$

where $P, Q$ are rational and integral functions of $x^{2}, P \pm Q x$ being each of them of the order $\frac{1}{2}(n-1)$, or, what is the same thing, $(1 \pm x)(P \pm Q x)^{2}$ being each of them of the order $n$; that is,

$$
n=4 p+1, \quad n=4 p+3,
$$

$$
\begin{array}{r}
\text { Order of } P \text { in } x^{2} \text { is } p, \quad p, \\
\# \quad Q \quad \# \quad p-1 ; \quad p ;
\end{array}
$$

whence in the first case the number of coefficients in $P$ and $Q$ is $(p+1)+p,=\frac{1}{2}(n+1)$, and in the second case the number is $(p+1)+(p+1),=\frac{1}{2}(n+1)$, as before. Taking

the formula is

$$
\begin{aligned}
& P=\alpha+\gamma x^{2}+\epsilon x^{4}+\ldots \\
& Q=\beta+\delta x^{2}+\zeta x^{4}+\ldots
\end{aligned}
$$

$$
\frac{1-y}{1+y}=\frac{1-x}{1+x}\left(\frac{\alpha-\beta x+\gamma x^{2}-\ldots}{\alpha+\beta x+\gamma x^{2}+\ldots}\right)^{2}
$$

the number of coefficients being as just explained. Starting herefrom I reproduce in a somewhat altered form the investigation in the Fundamenta Nova, as follows.

2. If the coefficients are such that the equation remains true when we therein change simultaneously $x$ into $\frac{1}{k x}$ and $y$ into $\frac{1}{\lambda y}$, then the variables $x, y$ will satisfy the differential equation

$$
\frac{M d y}{\sqrt{1-y^{2} \cdot 1-\lambda^{2} y^{2}}}=\frac{d x}{\sqrt{1-x^{2} \cdot 1-k^{2} x^{2}}}
$$


( $M$ a constant, the value of which, as will appear, is given by $\frac{1}{M}=1+\frac{2 \beta}{\alpha}$ ); and the problem of transformation is thus to find the coefficients so that the equation may remain true on the above simultaneous change of the values of $x, y$.

In fact, observing that the original equation and therefore the new equation are each satisfied on changing therein simultaneously $x, y$ into $-x,-y$, it follows that the equation may be written in the four forms

$$
\begin{array}{ll}
1-y=(1-x) A^{2}(\div), & 1+y=(1+x) B^{2}(\div), \\
1-\lambda y=(1-k x) C^{2}(\div), & 1+\lambda y=(1+k x) D^{2}(\div),
\end{array}
$$

the common denominator being, say $E$, where $A, B, C, D, E$ are all of them rational and integral functions of $x$; and this being so, the differential equation will be satisfied.

3. To develop the condition, observe that the assumed equation gives

$$
y=\frac{x\left(P^{2}+2 P Q+Q^{2} x^{2}\right)}{P^{2}+2 P Q x^{2}+Q^{2} x^{2}}, \quad=\frac{x \mathfrak{Y}}{\mathfrak{B}} \text { suppose, }
$$

where $\mathfrak{A}, \mathfrak{B}$ are functions each of them of the degree $\frac{1}{2}(n-1)$ in $x^{2}$. (Hence, if with Jacobi $\frac{1}{M}$ denotes the value $(y \div x)_{x=0}$, we have $\frac{1}{M}=\left(1+\frac{2 Q}{P}\right)_{x=0},=1+\frac{2 \beta}{\alpha}$, as mentioned.)

Suppose in general that $U$ being any integral function $\left(1, x^{2}\right)^{p}$, we have

$$
U^{*}=\left(k^{2} x^{2}\right)^{p}\left(1, \frac{1}{k^{2} x^{2}}\right)^{p}
$$

viz. let $U^{*}$ be what $U$ becomes when $x$ is changed into $\frac{1}{k x}$ and the whole multiplied by $\left(k^{2} x^{2}\right)^{p}$.

Let $y^{*}$ be the value of $y$ obtained by writing $\frac{1}{k x}$ for $x$; then, observing that in the expression for $y$ the degree of the numerator exceeds by unity that of the denominator, we have

whence

$$
y^{*}=\frac{1}{k x} \frac{\mathfrak{A}^{*}}{\mathfrak{B}^{*}}
$$

$$
y y^{*}=\frac{1}{k} \frac{\mathfrak{A R}^{*}}{\mathfrak{B B}^{*}}
$$

and the functions $\mathfrak{A}, \mathfrak{B}$ may be such that this shall be a constant value, $=\frac{1}{\lambda}$; viz. this will be the case if

$$
\frac{\lambda}{k}=\frac{\mathfrak{B} \mathfrak{B}^{*}}{\mathfrak{A} \mathfrak{A}^{*}},
$$

which being so, the required condition is satisfied. 
4. I shall ultimately, instead of $k, \lambda$ introduce Jacobi's $u, v(u=\sqrt[4]{k}, v=\sqrt[4]{\lambda})$; but it is for the present convenient to retain $k$, and instead of $\lambda$ to introduce the quantity $\Omega$ connected with it by the equation $\lambda=k \Omega^{2}$; or say the value of $\Omega$ is $=v^{2} \div u^{2}$. The modular equation in its standard form is an equation between $u, v$, which, as will appear, gives rise to an equation of the same order between $u^{2}, v^{2}$; and writing herein $v^{2}=\Omega u^{2}$, the resulting equation contains only integer powers of $u^{4}$, that is, of $k$, and we have an $\Omega k$-form of the modular equation, or say an $\Omega k$-modular equation, of the same order in $\Omega$ as the standard form is in $v$; these $\Omega k$-forms for $n=3,5,7,11$ will be given presently.

5. Suppose then, $\Omega$ being a constant, that we have identically

this implies

$$
\mathfrak{A}=\frac{1}{\Omega k^{\frac{1}{2}(n-1)}} \mathfrak{B}^{*} ;
$$

(In fact, if

$$
\mathfrak{B}=\frac{\Omega}{k^{\frac{1}{2}(n-1)}} \mathfrak{A}^{*}
$$

then

$$
\begin{aligned}
& \mathfrak{A}=a+c x^{2}+\ldots+q x^{n-3}+s x^{n-1}, \\
& \mathfrak{B}=b+d x^{2}+\ldots+r x^{n-3}+t x^{n-1},
\end{aligned}
$$

$$
\begin{aligned}
& \mathfrak{l}^{*}=s+q k^{2} x^{2}+\ldots+c k^{n-3} x^{n-3}+a k^{n-1} x^{n-1}, \\
& \mathfrak{B}^{*}=t+r k^{2} x^{2}+\ldots+d k^{n-3} x^{n-3}+b k^{n-1} x^{n-1},
\end{aligned}
$$

and the assumed equation gives

$$
a=\frac{1}{\Omega k^{\frac{1}{2}(n-1)}} t, \quad c=\frac{k^{2}}{\Omega k^{\frac{1}{2}(n-1)}} r, \ldots, q=\frac{k^{n-3}}{\Omega k^{\frac{1}{2}(n-1)}} d, \quad s=\frac{k^{n-1}}{\Omega k^{\frac{1}{2}(n-1)}} b,
$$

that is,

$$
b=\frac{\Omega}{k^{\frac{1}{2}(n-1)}} s, \quad d=\frac{\Omega k^{2}}{k^{\frac{1}{2}(n-1)}} q, \ldots, r=\frac{\Omega k^{n-3}}{k^{\frac{1}{2}(n-1)}} c, \quad t=\frac{\Omega k^{n-2}}{k^{\frac{1}{2}(n-1)}} a
$$

and therefore $\mathfrak{B}=\frac{\Omega}{k^{\frac{1}{2}(n-1)}} \mathfrak{I}^{*}$.)

From these equations $\frac{\mathfrak{B}^{*}}{\mathfrak{A O}^{*}}=\Omega^{2}$, that is, $=\frac{\lambda}{k}$, as it should be; so that $\Omega$ signifying as above, the required condition will be satisfied if only $\mathfrak{A}=\frac{1}{\Omega k^{\frac{1}{2}(n-1)}} \mathfrak{B} *$; or substituting for $\mathfrak{A}, \mathfrak{B}$ their values, if only

$$
\left(P^{2}+2 P Q x^{2}+Q^{2} x^{2}\right)^{*}=\Omega k^{\frac{1}{2}(n-1)}\left(P^{2}+2 P Q+Q^{2} x^{2}\right)
$$

where each side is a function of $x^{2}$ of the order $\frac{1}{2}(n-1)$, or the number of terms is $\frac{1}{2}(n+1)$, the several coefficients being obviously homogeneous quadric functions of the $\frac{1}{2}(n+1)$ coefficients of $P, Q$. We have thus $\frac{1}{2}(n+1)$ equations, each of the form $U=\Omega V$, where $U, V$ are given quadric functions of the coefficients of $P, Q$, say of the $\frac{1}{2}(n+1)$ coefficients $\alpha, \beta, \gamma, \delta, \& c$., and where $\Omega$ is indeterminate. 
6. We may from the $\frac{1}{2}(n+1)$ equations eliminate the $\frac{1}{2}(n-1)$ ratios $\alpha: \beta: \gamma: \ldots$, thus obtaining an equation in $\Omega$ (involving of course the parameter $k$ ) which is the $\Omega k$-modular equation above referred to; and then $\Omega$ denoting any root of this equation, the $\frac{1}{2}(n+1)$ equations give a single value for the set of ratios $\alpha: \beta: \gamma: \delta: \ldots$, so that the ratio of the functions $P, Q$ is determined, and consequently the value of $y$ as given by the equation

$$
\frac{1-y}{1+y}=\frac{(1-x)(P-Q x)^{2}}{(1+x)(P+Q x)^{2}} \text {, or } y=\frac{x\left(P^{2}+2 P Q+Q^{2} x^{2}\right)}{P^{2}+2 P Q x^{2}+Q^{2} x^{2}} .
$$

The entire problem thus depends on the solution of the system of $\frac{1}{2}(n+1)$ equations,

$$
\left(P^{2}+2 P Q x^{2}+Q^{2} x^{2}\right)^{*}=\Omega k^{\frac{1}{2}(n-1)}\left(P^{2}+2 P Q+Q^{2} x^{2}\right) .
$$

The $\Omega k$-Modular Equations, $n=3,5,7,11$. Article No. 7 .

7. For convenience of reference, and to fix the ideas, I give these results, calculated, as above explained, from the standard or $u v$-forms.

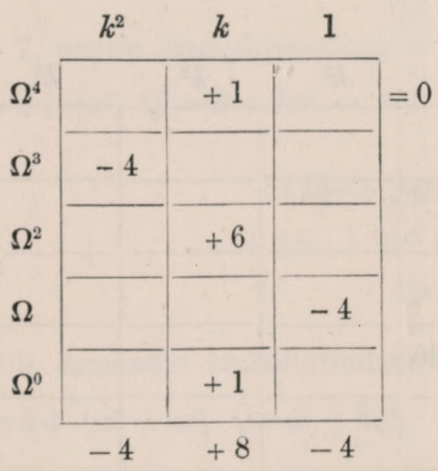

$n=3:$

$$
\Omega=1 \text {, we have }-4(k-1)^{2}=0 \text {. }
$$

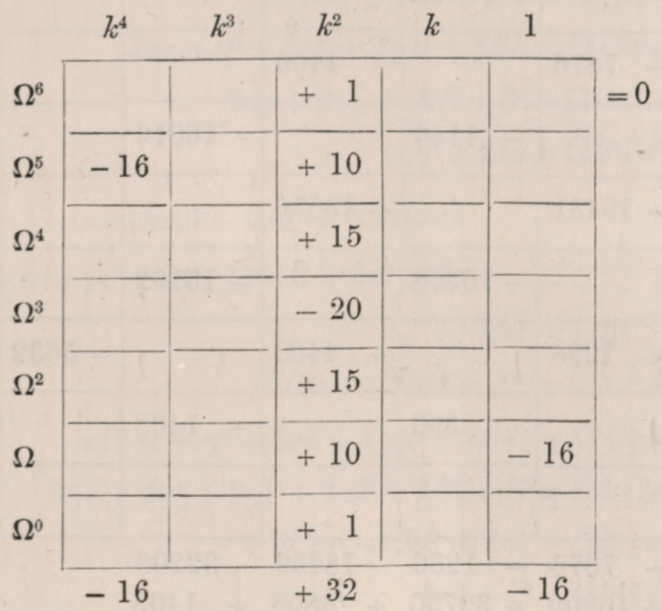

$n=5:$

$\Omega=1$, we have $-16\left(k^{2}-1\right)^{2}=0$. 
118 A MEMOIR ON THE TRANSFORMATION OF ELLIPTIC FUNCTIONS.
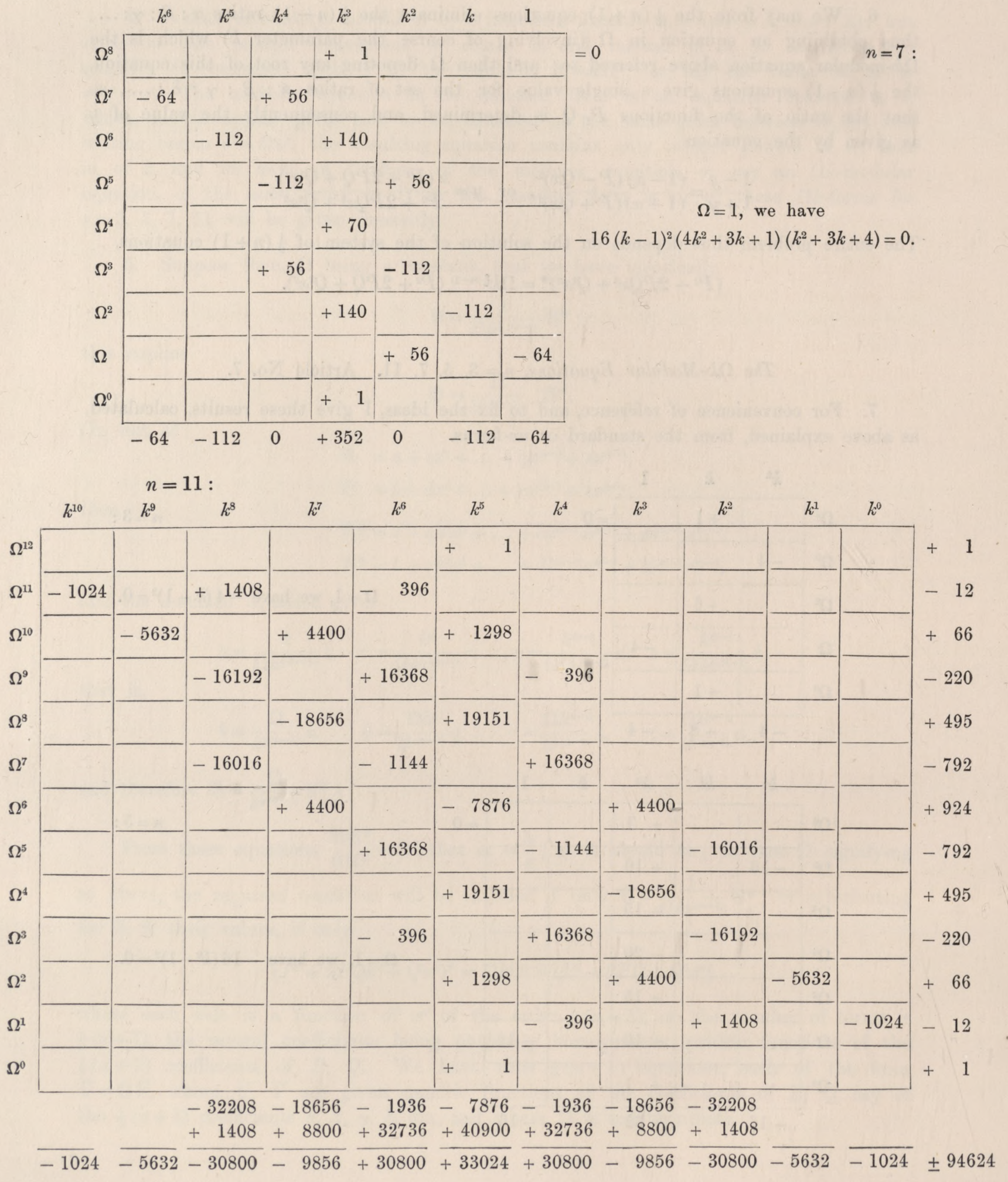
Equation-systems for the cases $n=3,5,7,9,11$. Art. Nos. 8 to 10 .

8. $u=3$, cubic transformation. $\quad k=u^{4}, \Omega=\frac{v^{2}}{u^{2}}$ (here and in the other cases).

$P=\alpha, Q=\beta$. The condition here is

$$
k^{2} x^{2} \alpha^{2}+\left(2 \alpha \beta+\beta^{2}\right)=\Omega k\left\{\left(\alpha^{2}+2 \alpha \beta\right)+\beta^{2} x^{2}\right\},
$$

and the system of equations thus is

$$
\begin{aligned}
k \alpha^{2} & =\Omega \beta^{2}, \\
2 \alpha \beta+\beta^{2} & =\Omega k\left(\alpha^{2}+2 \alpha \beta\right),
\end{aligned}
$$

and similarly in the other cases; for these it will be enough to write down the equation-systems.

$n=5$, quintic transformation.

$P=\alpha+\gamma x^{2}, Q=\beta$.

$$
\begin{aligned}
k^{2} \alpha^{2} & =\Omega \gamma^{2}, \\
2 \alpha \gamma+2 \alpha \beta+\beta^{2} & =\Omega\left(2 \alpha \gamma+2 \beta \gamma+\beta^{2}\right), \\
\gamma^{2}+2 \beta \gamma & =\Omega k^{2}\left(\alpha^{2}+2 \alpha \beta\right) .
\end{aligned}
$$

$n=7$, septic transformation.

$P=\alpha+\gamma x^{2}, Q=\beta+\delta x^{2}$.

$$
\begin{aligned}
k^{3} \alpha^{2} & =\Omega \delta^{2} \\
k\left(2 \alpha \gamma+2 \alpha \beta+\beta^{2}\right) & =\Omega\left(\gamma^{2}+2 \gamma \delta+2 \beta \delta\right) \\
\gamma^{2}+2 \beta \gamma+2 \alpha \delta+2 \beta \delta & =\Omega k\left(2 \alpha \gamma+2 \beta \gamma+2 \alpha \delta+\beta^{2}\right) \\
\delta^{2}+2 \gamma \delta & =\Omega k^{3}\left(\alpha^{2}+2 \alpha \beta\right)
\end{aligned}
$$

$n=9$, enneadic transformation.

$$
\begin{aligned}
P=\alpha+\gamma x^{2}+\epsilon x^{4}, Q=\beta+\delta x^{2} . & k^{4} \alpha^{2}=\Omega \epsilon^{2}, \\
k^{2}\left(2 \alpha \gamma+2 \alpha \beta+\beta^{2}\right) & =\Omega\left(2 \gamma \epsilon+2 \epsilon \delta+\delta^{2}\right), \\
2 \alpha \epsilon+\gamma^{2}+2 \alpha \delta+2 \gamma \beta+2 \beta \delta & =\Omega\left(2 \alpha \epsilon+\gamma^{2}+2 \gamma \delta+2 \epsilon \beta+2 \beta \delta\right), \\
2 \gamma \epsilon+2 \gamma \delta+2 \epsilon \beta+\delta^{2} & =\Omega k^{2}\left(2 \alpha \gamma+2 \alpha \delta+2 \gamma \beta+\beta^{2}\right), \\
\epsilon^{2}+2 \delta \epsilon & =\Omega k^{4}\left(\alpha^{2}+2 \alpha \beta\right) .
\end{aligned}
$$

$n=11$, endecadic transformation.

$$
\begin{aligned}
P=\alpha+\gamma x^{2}+\epsilon x^{4}, Q=\beta+\delta x^{2}+\zeta x^{4} . & k^{5} \alpha^{2}=\Omega \zeta^{2}, \\
k^{3}\left(2 \alpha \gamma+2 \alpha \beta+\beta^{2}\right) & =\Omega\left(\epsilon^{2}+2 \epsilon \zeta+2 \delta \zeta\right), \\
k\left(2 \alpha \epsilon+\gamma^{2}+2 \alpha \delta+2 \gamma \beta+2 \beta \delta\right) & =\Omega\left(2 \gamma \epsilon+2 \gamma \zeta+2 \epsilon \delta+2 \beta \delta+\delta^{2}\right), \\
2 \gamma \epsilon+2 \alpha \zeta+2 \gamma \delta+2 \epsilon \beta+2 \beta \zeta+\delta^{2} & =\Omega k\left(2 \alpha \epsilon+\gamma^{2}+2 \alpha \zeta+2 \gamma \delta+2 \epsilon \beta+2 \beta \delta\right), \\
\epsilon^{2}+2 \gamma \zeta+2 \epsilon \delta+2 \delta \zeta & =\Omega k^{3}\left(2 \alpha \gamma+2 \alpha \delta+2 \gamma \beta+\beta^{2}\right), \\
2 \epsilon \zeta+\zeta^{2} & =\Omega k^{5}\left(\alpha^{2}+2 \alpha \beta\right) .
\end{aligned}
$$

And so on. 
9. It will be noticed that if the coefficients of $P+Q x$ taken in order are

$$
\alpha, \beta, \ldots, \rho, \sigma
$$

then in every case the first and last equations are

$$
\begin{aligned}
& k^{\frac{1}{2}(n-1)} \alpha^{2}=\Omega \sigma^{2}, \\
& 2 \rho \sigma+\sigma^{2}=\Omega k^{\frac{1}{2}(n-1)}\left(\alpha^{2}+2 \alpha \beta\right) .
\end{aligned}
$$

Putting in the first of these $k=u^{4}, \Omega=\frac{v^{2}}{u^{2}}$, the equation becomes

$$
u^{2 n} \alpha^{2}=v^{2} \sigma^{2}
$$

where each side is a perfect square; and in extracting the square root we may without loss of generality take the roots positive, and write $u^{n} \alpha=v \sigma$.

This speciality, although it renders it proper to employ ultimately $u, v$ in place of $k, \Omega$, produces really no depression of order (viz. the $\Omega k$-form of the modular equation is found to be of the same order in $\Omega$ that the standard or $u v$-form is in $v$ ), and is in another point of view a disadvantage, as destroying the uniformity of the several equations: in the discussion of order I consequently retain $\Omega, k$. Ultimately these are to be replaced by $u, v$; the change in the equation-systems is so easily made that it is not necessary here to write them down in the new form in $u, v$.

10. The case $\alpha=0$ has to be considered in the discussion of order, but we have thus only solutions which are to be rejected; in the proper solutions $\alpha$ is not $=0$, and it may therefore for convenience be taken to be $=1$. We have then $\sigma=u^{n} \div v$. The last equation becomes therefore

$$
\frac{u^{n}}{v}\left(2 \rho+\frac{u^{n}}{v}\right)=\frac{v^{2}}{u^{2}} u^{2 n-2}(1+2 \beta)
$$

or recollecting that $\beta$ is connected with the multiplier $M$ by the relation $\frac{1}{M}=1+2 \beta$, that is,

$$
2 \beta=\frac{1}{M}-1
$$

and substituting for $1+2 \beta$ its value, the equation becomes

$$
2 \rho=v^{3} u^{n-4}\left(\frac{1}{M}-\frac{u^{4}}{v^{4}}\right)
$$

that is, the first and the last coefficients are $1, \frac{u^{n}}{v}$, and the second and the penultimate coefficients are each expressed in terms of $v, M$. The cases $n=3, n=5$ are so far peculiar, that the only coefficients are $\alpha, \beta$, or $\alpha, \beta, \gamma$; in the next case $n=7$, the only coefficients are $\alpha, \beta, \gamma, \delta$, and we have in this case all the coefficients expressed as above. 
The $\Omega k$-form-Order of the Systems. Art. Nos. 11 to 22.

11. In the general case, $n$ an odd number, we have $\Omega$ and $\frac{1}{2}(n+1)$ coefficients connected by a system of $\frac{1}{2}(n+1)$ equations of the form

$$
\Omega=\frac{U}{U^{\prime}}=\frac{V}{V^{\prime}}=\ldots,
$$

where $U, V, \ldots, U^{\prime}, V^{\prime}, \ldots$ are given quadric functions of the coefficients. Omitting the $(\Omega=)$, there remains a system of $\frac{1}{2}(n-1)$ equations of the form $\frac{U}{U^{\prime}}=\frac{V}{V^{\prime}}=\ldots$, or say

$$
\left(\begin{array}{ccc}
U, & V, & W, \ldots \\
U^{\prime}, & V^{\prime}, & W^{\prime}, \ldots
\end{array} \mid=0,\right.
$$

which determine the ratios $\alpha: \beta: \gamma: \ldots$ of the coefficients; and to each set of ratios there corresponds a single value of $\Omega$. The order of the system, or number of sets of ratios, is $=\frac{1}{2}(n+1) \cdot 2^{\frac{1}{2}(n-1)},=(n+1) \cdot 2^{\frac{1}{2}(n-3)}$; and this is consequently the number of values of $\Omega$, or the order of the equation for the determination of $\Omega$; viz. but for reduction, the order in $\Omega$ of the $\Omega k$-modular equation would be $=(n+1) \cdot 2^{\frac{1}{2}(n-3)}$. In the case $n=3$, this is $=4$, which is right, but for any larger value of $n$ the order is far too high ; in fact, assuming (as the case is) that the order is equal to the order in $v$ of the $u v$-form, the order should for a prime value of $n$ be $=n+1$, and for a composite value not containing any square factor be $=$ the sum of the divisors of $n$. I do not attempt a general investigation, but confine myself to showing in what manner the reductions arise.

12. I will first consider the cubic transformation; here, writing for convenience $\frac{\alpha}{\beta}=\theta$, the equations give

and

$$
\frac{k \theta^{2}}{2 \theta+1}=\frac{1}{k\left(\theta^{2}+2 \theta\right)}, \text { that is, } k^{2} \theta^{3}(\theta+2)-(2 \theta+1)=0
$$

$$
k \theta^{2}=\Omega .
$$

The equation in $\theta$ gives $\left(k^{2} \theta^{4}-1\right)^{2}-4 \theta^{2}\left(k^{2} \theta^{2}-1\right)^{2}=0$, and we have thence

that is,

$$
k\left(\Omega^{2}-1\right)^{2}-4 \Omega(k \Omega-1)^{2}=0,
$$

$$
k \Omega^{4}-4 k^{2} \Omega^{3}+6 k \Omega^{2}-4 \Omega+k=0,
$$

the modular equation; and then $k^{2} \theta^{4}-1+2 \theta\left(k^{2} \theta^{2}-1\right)=0$, that is, $\Omega^{2}-1+2 \theta(k \Omega-1)=0$, or $2 \theta=-\frac{\Omega^{2}-1}{k \Omega-1}$, which is $=\frac{2 \alpha}{\beta}$, say we have $\alpha=\Omega^{2}-1, \beta=2(1-k \Omega)$; consequently

which completes the theory.

$$
\begin{gathered}
\frac{1-y}{1+y}=\frac{1-x}{1+x}\left\{\frac{\Omega^{2}-1+2(k \Omega-1) x}{\Omega^{2}-1-2(k \Omega-1) x}\right\}^{2}, \\
\lambda=\Omega^{2} k \text {, and } \frac{1}{M}=\frac{\alpha^{2}+2 \alpha \beta}{\alpha^{2}}=\frac{\theta+2}{\theta}=\frac{\Omega^{2}-4 k \Omega+3}{\Omega^{2}-1},
\end{gathered}
$$

c. IX. 
13. Reproducing for this case the general theory, it appears à priori that $\Omega$ is determined by a quartic equation; in fact, from the original equations eliminating $\Omega$, we have an equation

$$
\left|\begin{array}{ll}
U, & V \\
U^{\prime}, & V^{\prime}
\end{array}\right|=0,
$$

where $U, U^{\prime}, V, V^{\prime}$ are quartic functions of $\alpha, \beta$; that is, the ratio $\alpha: \beta$ has four values, and to eaeh of these there corresponds a single value of $\Omega$; viz. $\Omega$ is determined by a quartic equation.

14. Considering next the case $n=5$, the quintic transformation; the elimination of $\Omega$ gives the equations

$$
\frac{U}{U^{\prime}}=\frac{V}{V^{\prime}}=\frac{W}{W^{\prime}},
$$

where $U, U^{\prime}$, \&c. are all quadric functions of $\alpha, \beta, \gamma$. We have thence $4.4-2.2,=12$ sets of values of $\alpha: \beta: \gamma$; viz. considering $\alpha, \beta, \gamma$ as coordinates in plano, the curves $U V^{\prime}-U^{\prime} V=0, U W^{\prime}-U^{\prime} W=0$ are quartic curves intersecting in 16 points; but among these are included the four points $U=0, U^{\prime}=0$ (in fact, the point $\alpha=0, \gamma=0$ four times), which are not points of the curve $V W^{\prime}-V^{\prime} W=0$; there remain therefore $16-4,=12$ intersections, agreeing with the general value $(n+1) \cdot 2^{\frac{1}{2}(n-3)}$. Hence $\Omega$ is in the first instance determined by an equation of the order 12; but the proper order being $=6$, there must be a factor of the order 6 to be rejected. To explain this and to determine the factor, observe that the equations in question are

$$
\begin{aligned}
& k^{2} \alpha^{2}\left(2 \alpha \gamma+2 \beta \gamma+\beta^{2}\right)-\gamma^{2}\left(2 \alpha \gamma+2 \alpha \beta+\beta^{2}\right)=0, \\
& k^{4} \alpha^{3}(\alpha+2 \beta) \quad-\gamma^{3}(\gamma+2 \beta)
\end{aligned}
$$

at the point $\alpha=0, \gamma=0$, the first of these has a double point, the second a triple point; or there are at the point in question 6 intersections; but 4 of these are the points which give the foregoing reduction $16-4=12$; we have thus the point $\alpha=0$, $\gamma=0$, counting twice among the twelve points. Writing in the two equations $\beta=0$, the equations become $k^{2} \alpha^{3} \gamma-\alpha \gamma^{3}=0, k^{4} \alpha^{4}-\gamma^{4}=0$, viz. these will be satisfied if $k^{2} \alpha^{2}-\gamma^{2}=0$, that is, the curves pass through each of the two points $(\beta=0, \gamma= \pm k \alpha)$, and these values satisfy (as in fact they should) the third equation

$$
k^{2}\left(2 \alpha \gamma+2 \alpha \beta+\beta^{2}\right) \alpha(\alpha+2 \beta)-\gamma(\gamma+2 \beta)\left(2 \alpha \gamma+2 \beta+\beta^{2}\right)=0 .
$$

It is moreover easily shown that the three curves have at each of the points in question a common tangent; viz. taking $A, B, C$ as current coordinates, the tangent at the point $(\alpha, \beta, \gamma)$ of the second curve has for its equation

$$
A\left(2 \alpha^{3}+3 \alpha^{2} \beta\right) k^{4}+B\left(k^{4} \alpha^{3}-\gamma^{3}\right)-C\left(2 \gamma^{3}+3 \gamma^{2} \beta\right)=0
$$

and for $\beta=0, \gamma= \pm k \alpha$, this becomes $2 k A+B(k \mp 1) \mp 2 C=0$, viz. this is the line from the point $(\beta=0, \gamma= \pm k \alpha)$ to the point $(1,-2,1)$. And similarly for the other two curves we find the same equation for the tangent. 
Hence among the 12 points are included the point $(\gamma=0, \alpha=0)$ twice, and the points $(\beta=0, \gamma= \pm k \alpha)$ each twice: we have thus a reduction $=6$.

15. Writing in the equations $\gamma=0, \alpha=0$, the first and third are satisfied identically, and the second becomes $\beta^{2}=\Omega \beta^{2}$, that is, the equations give $\Omega=1$; writing $\beta=0$, they become

$$
k^{2} \alpha^{2}=\Omega \gamma^{2}, \quad \alpha \gamma=\Omega \alpha \gamma, \quad \gamma^{2}=\Omega k^{2} \alpha^{2},
$$

viz. putting herein $\gamma^{2}=k^{2} \alpha^{2}$, the equations again give $\Omega=1$; hence the factor of the order 6 is $(\Omega-1)^{6}$, and the equation of the twelfth order for the determination of $\Omega$ is

$$
(\Omega-1)^{6}\left\{(\Omega, 1)^{6}\right\}=0,
$$

where $(\Omega, 1)^{6}=0$ is the $\Omega k$-modular equation above written down.

16. Reverting to the equation

$$
\frac{1-y}{1+y}=\frac{(1-x)(P-Q x)^{2}}{(1+x)(P+Q x)^{2}}
$$

it is to be observed that for $\alpha=0, \gamma=0$, that is, $P=0$, this becomes simply $y=x$, which is the transformation of the order 1 ; the corresponding value of the modulus $\lambda$ is $\lambda=k$, and the equation $\lambda=\Omega^{2} k$ then gives $\Omega^{2}=1$, which is replaced by $\Omega-1=0$.

If in the same equation we write $\beta=0$, that is, $Q=0$, then (without any use of the equation $\gamma^{2}=k^{2} \alpha^{2}$ ) we have $y=x$, the transformation of the order 1 ; but although this is so, the fundamental equation

$$
\left(P^{2}+2 P Q x^{2}+Q^{2} x^{2}\right)^{*}=\Omega k^{2}\left(P^{2}+2 P Q+Q^{2} x^{2}\right),
$$

which, putting therein $Q=0$, becomes $\left(P^{2}\right)^{*}=\Omega k^{2} P^{2}$, that is, $\left(k^{2} x^{2} \alpha+\gamma\right)^{2}=\Omega k^{2}\left(\alpha+\gamma x^{2}\right)^{2}$, is not satisfied by the single relation $\Omega-1=0$, but necessitates the further relation $\gamma^{2}=k^{2} \alpha^{2}$.

The thing to be observed is that the extraneous factor $(\Omega-1)^{6}$, equated to zero, gives for $\Omega$ the value $\Omega=1$ corresponding to the transformation $y=x$ of the order 1 .

17. Considering next $n=7$, the septic transformation; we have here between $\alpha, \beta, \gamma, \delta$ a fourfold relation of the form

$$
\left(\begin{array}{llll}
U, & V, & W, & Z \\
U^{\prime}, & V^{\prime}, & W^{\prime}, & Z^{\prime}
\end{array}\right)=0
$$

where, as before, $U, U^{\prime}$, \&c. are quadric functions, and the number of solutions is here $8.2^{2},=32$; to each of these corresponds a single value of $\Omega$, or $\Omega$ is in the first instance determined by an equation of the order 32 . But the order of the modular equation is $=8$; or representing this by $\left\{(\Omega, 1)^{8}\right\}=0$, the equation must be

$$
(\Omega, 1)^{24}\left\{(\Omega, 1)^{8}\right\}=0,
$$

viz. there must be a special factor of the order 24 . 
18. One way of satisfying the equations is to write therein $\alpha=0, \delta=0$; the equations thus become

or putting $\beta, \gamma=\alpha^{\prime}, \beta^{\prime}$,

$$
\begin{aligned}
k \beta^{2} & =\Omega \gamma^{2} \\
\gamma^{2}+2 \beta \gamma & =\Omega k\left(2 \beta \gamma+\beta^{2}\right) \\
k \alpha^{\prime 2} & =\Omega \beta^{\prime 2} \\
\beta^{\prime 2}+2 \alpha^{\prime} \beta^{\prime} & =\Omega k\left(2 \alpha^{\prime} \beta^{\prime}+\alpha^{\prime 2}\right),
\end{aligned}
$$

which (with $\alpha^{\prime}, \beta^{\prime}$ instead of $\alpha, \beta$ ) are the very equations which belong to the cubic transformation; hence a factor is $\left\{(\Omega, 1)^{4}\right\}$.

Observe that for the values in question $\alpha=0, \delta=0, P=\beta^{\prime} x^{2}, Q=\alpha^{\prime}$,

and therefore

$$
(P \pm Q x)^{2}=x^{2}\left(\alpha^{\prime} \pm \beta^{\prime} x\right)^{2},=x^{2}\left(P^{\prime} \pm Q^{\prime} x\right)^{2}, \text { if } P^{\prime}=\alpha^{\prime}, Q^{\prime}=\beta^{\prime},
$$

$$
\frac{1-y}{1+y}=\frac{1-x}{1+x}\left(\frac{P^{\prime}-Q^{\prime} x}{P^{\prime}+Q^{\prime} x}\right)^{2}
$$

which is the formula for a cubic transformation.

19. The equations may also be satisfied by writing therein $\gamma=k \alpha, \delta=k \beta$; in fact, substituting these values, they become

$$
\begin{aligned}
k^{3} \alpha^{2} & =\Omega k^{2} \beta^{2} \\
2 k^{2} \alpha^{2}+k\left(2 \alpha \beta+\beta^{2}\right) & =\Omega k^{2}\left(\alpha^{2}+2 \alpha \beta\right)+2 \Omega k \beta^{2}, \\
k^{2} \alpha^{2}+2 k\left(\beta^{2}+2 \alpha \beta\right) & =2 \Omega k^{2}\left(\alpha^{2}+2 \alpha \beta\right)+\Omega k \beta^{2}, \\
k^{2}\left(\beta^{2}+2 \alpha \beta\right) & =\Omega k^{3}\left(\alpha^{2}+2 \alpha \beta\right)
\end{aligned}
$$

the first and last of these are

$$
\begin{aligned}
k \alpha^{2} & =\Omega \beta^{2}, \\
\beta^{2}+2 \alpha \beta & =\Omega k\left(\alpha^{2}+2 \alpha \beta\right),
\end{aligned}
$$

which being satisfied the second and third equations are satisfied identicaliy; and these are the formulæ for a cubic transformation; that is, we again have the factor $\left\{(\Omega, 1)^{4}\right\}$.

Observe that for the values in question $\gamma=k \alpha, \delta=k \beta$, we have $P=\alpha\left(1+k x^{2}\right)$, $Q=\beta\left(1+k x^{2}\right)$; so that, writing $P^{\prime}=\alpha, Q^{\prime}=\beta$, we have for $y$ the value

$$
\frac{1-y}{1+y}=\frac{(1-x)\left(P^{\prime}-Q^{\prime} x\right)^{2}}{(1+x)\left(P^{\prime}+Q^{\prime} x\right)^{2}}
$$

which is the formula for a cubic transformation.

20. It is important to notice that we cannot by writing $\alpha=0$ or $\delta=0$ reduce the transformation to a quintic one; in fact, the equation $k^{3} \alpha^{2}=\Omega \delta^{2}$ shows that if either of these equations is satisfied the other is also satisfied; and we have then the foregoing case $\alpha=0, \delta=0$, giving not a quintic but a cubic transformation.

And for the same reason we cannot by writing $\alpha=0, \beta=0, \gamma=0$ or $\beta=0, \gamma=0$, $\delta=0$ reduce the transformation to the order 1 . There is thus no factor $\Omega-1$. 
21. As regards the non-existence of the factor $\Omega-1$, I further verify this by writing in the equations $\Omega=1$; they thus become

$$
\begin{aligned}
k^{3} \alpha^{2} & =\delta^{2} \\
k\left(2 \alpha \gamma+2 \alpha \beta+\beta^{2}\right) & =\gamma^{2}+2 \gamma \delta+2 \beta \delta \\
\gamma^{2}+2 \beta \gamma+2 \alpha \delta+2 \beta \delta & =k\left(2 \alpha \gamma+2 \beta \gamma+2 \alpha \delta+\beta^{2}\right), \\
\delta^{2}+2 \gamma \delta & =k^{3}\left(\alpha^{2}+2 \alpha \beta\right),
\end{aligned}
$$

which it is to be shown cannot be satisfied in general, but only for certain values of $k$.

Reducing the last equation, this is $\gamma \delta=k^{3} \alpha \beta$, which, combined with the first, gives $\alpha \gamma=\beta \delta$; and if for convenience we assume $\alpha=1$, and write also $\theta= \pm \sqrt{k}$ (that is, $k=\theta^{2}$ ), then the values of $\alpha, \beta, \gamma, \delta$ are $\alpha=1, \beta=\gamma \theta^{-3}, \gamma=\gamma, \delta=\theta^{3}$; which values, substituted in the second and the third equations, give two equations in $\gamma, \theta$; and from these, eliminating $\gamma$, we obtain an equation for the determination of $\theta$, that is, of $k$. In fact, the second equation gives

$$
\theta^{2}\left(2 \gamma+2 \gamma \theta^{-3}+\gamma^{2} \theta^{-6}\right)=\gamma^{2}+2 \gamma \theta^{3}+2 \gamma
$$

or, dividing by $\gamma$ and reducing,

that is,

$$
\gamma\left(1-\theta^{4}\right)=2 \theta^{3}\left(\theta^{2}-1\right)\left(\theta^{2}-\theta+1\right)
$$

$$
\gamma\left(1+\theta^{2}\right)=-2 \theta^{3}\left(\theta^{2}-\theta+1\right)
$$

or, as this may also be written,

that is,

$$
\left(\gamma+\theta^{3}\right)\left(1+\theta^{2}\right)=-\theta^{3}(\theta-1)^{2}
$$

$$
\gamma+\theta^{3}=\frac{-\theta^{3}(\theta-1)^{2}}{\theta^{2}+1}
$$

Moreover the third equation gives

that is,

$$
\gamma^{2}+2 \gamma^{2} \theta^{-3}+2 \theta^{3}+2 \gamma=\theta^{2}\left(2 \gamma+2 \gamma^{2} \theta^{-3}+2 \theta^{3}+\gamma^{2} \theta^{-6}\right),
$$

or dividing by $\theta^{2}-1$, it is

$$
\gamma^{2}\left(\theta^{4}-2 \theta^{3}+2 \theta-1\right)-2\left(\gamma+\theta^{3}\right) \theta^{4}\left(\theta^{2}-1\right)=0 ;
$$

whence also

$$
\gamma^{2}(\theta-1)^{2}=2 \theta^{4}\left(\gamma+\theta^{3}\right)
$$

$$
\gamma^{2}=\frac{-2 \theta^{7}}{\theta^{2}+1}
$$

Also

wherefore

$$
4 \theta^{6}\left(\theta^{2}-\theta+1\right)^{2}=\gamma^{2}\left(\theta^{2}+1\right)^{2},
$$

or

$$
2\left(\theta^{2}-\theta+1\right)^{2}=-\theta\left(\theta^{2}+1\right) \text { or } 2\left(\theta^{2}-\theta+1\right)^{2}+\theta\left(\theta^{2}+1\right)=0 \text {, }
$$

that is,

or finally

$$
\theta\left(\theta^{2}+1\right)^{2}+2\left(\theta^{2}-\theta+1\right)^{2}=0
$$

$$
2 \theta^{4}-3 \theta^{3}+6 \theta^{2}-3 \theta+2=0,
$$

$$
\left(2 \theta^{2}-\theta+1\right)\left(\theta^{2}-\theta+2\right)=0 \text {. }
$$


We have thus $\left(2 \theta^{2}+1\right)^{2}=\theta^{2}$, that is, $4 \theta^{4}+3 \theta^{2}+1=0$ or $4 k^{2}+3 k+1=0$, or else $\left(\theta^{2}+2\right)^{2}=\theta^{3}$, that is, $\theta^{4}+3 \theta^{2}+4=0$ or $k^{2}+3 k+4=0$; viz. the equation in $k$ is

$$
\left(4 k^{2}+3 k+1\right)\left(k^{2}+3 k+4\right)=0
$$

these being in fact the values of $k$ given by the modular equation on putting therein $\Omega=1$.

The equation of the order 32 thus contains the factor $\left\{(\Omega, 1)^{4}\right\}$ at least twice, and it does not contain either the factor $\Omega-1$, or the factor $\left\{(\Omega, 1)^{6}\right\}$ belonging to the quintic transformation; it may be conjectured that the factor $\left\{(\Omega, 1)^{4}\right\}$ presents itself six times, and that the form is

$$
\left\{(\Omega, 1)^{4}\right\}^{6}(\Omega, 1)^{8}=0 ;
$$

but I am not able to verify this, and I do not pursue the discussion further.

22. The foregoing considerations show the grounds of the difficulty of the purely algebraical solution of the problem; the required results, for instance the modular equation, are obtained not in the simple form, but accompanied with special factors of high order. The transcendental theory affords the means of obtaining the results in the proper form without special factors; and I proceed to develop the theory, reproducing the known results as to the modular and multiplier equations, and extending it to the determination of the transformation-coefficients $\alpha, \beta, \ldots$

\section{The Modular Equation. Art. Nos. 23 to 28.}

23. Writing, as usual, $q=e^{-\frac{\pi K^{\prime}}{K}}$, we have $u$, a given function of $q$, viz.

$$
\begin{aligned}
u & =\sqrt{2} q^{\frac{1}{8}} \frac{1+q^{2} \cdot 1+q^{4} \cdot 1+q^{6} \cdot .}{1+q \cdot 1+q^{3} \cdot 1+q^{5} \cdot} \\
& =\sqrt{2} q^{\frac{1}{8}}\left(1-q+2 q^{2}-3 q^{3}+4 q^{4}-6 q^{5}+9 q^{6}-12 q^{7}+\ldots\right) \\
& =\sqrt{2} q^{\frac{1}{8}} f(q) \text { suppose }
\end{aligned}
$$

and this being so, the several values of $v$ and of the other quantities in question are all given in terms of $q$.

The case chiefly considered is that of $n$ an odd prime; and unless the contrary is stated it is assumed that this is so. We have then $n+1$ transformations corresponding to the same number $n+1$ of values of $v$; these may be distinguished as $v_{0}, v_{1}, v_{2}, \ldots, v_{n}$; viz. writing $\alpha$ to denote an imaginary $n$th root of unity, we have

$$
\begin{aligned}
& v_{0}=(-)^{\frac{n^{2}-1}{8}} \sqrt{2} q^{\frac{n}{8}} f\left(q^{n}\right), \quad v_{1}=\sqrt{2}\left(\alpha q^{\frac{1}{n}}\right)^{\frac{1}{8}} f\left(\alpha q^{\frac{1}{n}}\right), \quad v_{2}=\sqrt{2}\left(\alpha^{2} q^{\frac{1}{n}}\right)^{\frac{1}{8}} f\left(\alpha^{2} q^{\frac{1}{n}}\right), \quad \& c ., \\
& v_{n}=\quad \sqrt{2} q^{\frac{1}{8 n}} f\left(q^{\frac{1}{n}}\right) \text {. } \\
& \text { (Observe }(-)^{\frac{n^{2}-1}{8}}=+ \text { for } n=8 p \pm 1,- \text { for } n=8 p \pm 3 \text {.) }
\end{aligned}
$$


The occurrence of the fractional exponent $\frac{1}{8}$ is, as will appear, a circumstance of great importance; and it will be convenient to introduce the term "octicity," viz. an expression of the form $q^{\frac{f}{8}} F^{\prime}(q)(f=0$, or a positive integer not exceeding $7, F(q)$ a rational function of $q$ ) may be said to be of the octicity $f$.

24. The modular equation is of course

say this is

$$
\left(v-v_{0}\right)\left(v-v_{1}\right) \ldots\left(v-v_{n}\right)=0 ;
$$

$$
v^{n+1}-A v^{n}+B v^{n-1}-\ldots=0,
$$

so that $A=\Sigma v_{0}, B=\Sigma v_{0} v_{1}$, \&c. In the development of these expressions, the terms having a fractional exponent, with denominator $n$, would disappear of themselves, as involving symmetrically the several $n$th roots of unity; and each coefficient would be of the form $q^{8} F(q), F$ a rational and integral function of $q$. It is moreover easy to see that, for the several coefficients $A, B, C, \ldots \ldots, g$ will denote the positive residue (mod. 8) of $n, 2 n, 3 n, \ldots$ respectively.

Hence assuming, as the fact is, that these coefficients are severally rational and integral functions of $q$, it follows that the form is

$$
a u^{g}+b u^{g+8}+c u^{g+16}+\ldots \ldots,
$$

$g$ having the foregoing values for the several coefficients respectively. And it being known that the modular equation is as regards $u$ of the order $=n+1$, there is a known limit to the number of terms in the several coefficients respectively. We have thus for each coefficient an identity of the form

$$
A=a u^{g}+b u^{g+8}+\ldots \ldots,
$$

where $A$ and $u$ being each of them given in terms of $q$, the values of the numerical coefficients $a, b, \ldots$ can be determined; and we thus arrive at the modular equation.

25. It is in effect in this manner that the modular equations are calculated in Sohnke's Memoir. Various relations of symmetry in regard to $(u, v)$ and other known properties of the modular equation are made use of in order to reduce the number of the unknown coefficients to a minimum; and (what in practice is obviously an important simplification) instead of the coefficients $\Sigma v_{0}, \Sigma v_{0} v_{1}$, \&c., it is the sums of powers $\Sigma v_{0}, \Sigma v_{0}^{2}$, \&c., which are compared with their expressions in terms of $u$, in order to the determination of the unknown numerical coefficients $a, b, \ldots$ The process is a laborious one (although less so than perhaps might beforehand have been imagined), involving very high numbers; it requires the development up to high powers of $q$, of the high powers of the before-mentioned function $f(q)$; and Sohnke gives a valuable Table, which I reproduce here; adding to it the three columns which relate to $\phi q$. 


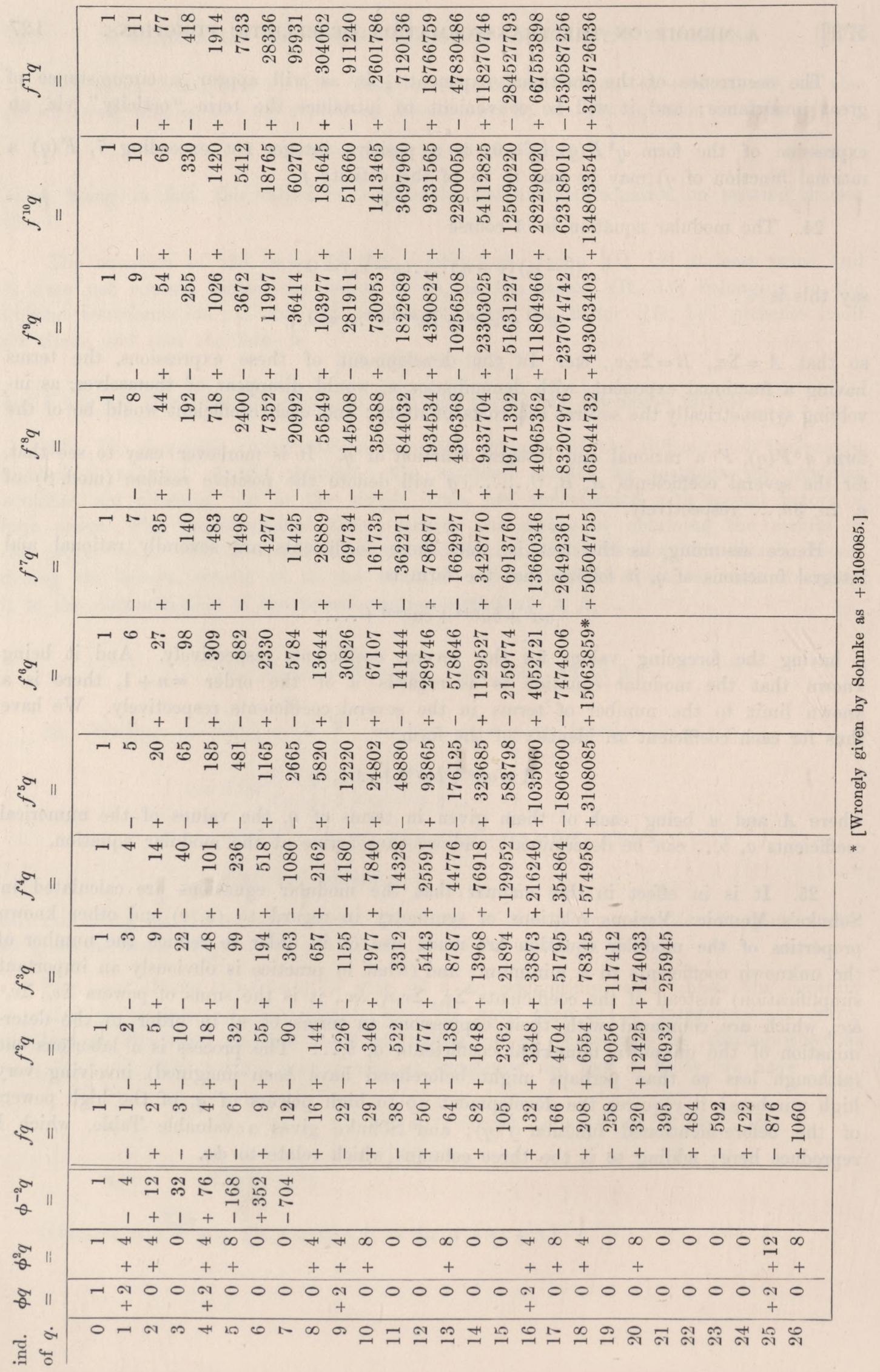


26. I give from Sohnke the series of modular equations, adding those for the composite cases $n=9$ and $n=15$, as to which see the remarks which follow the Table.

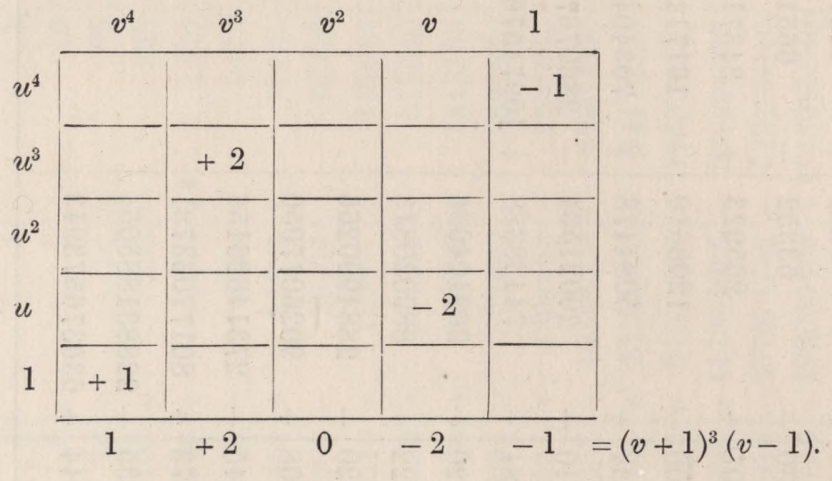

$n=3$.
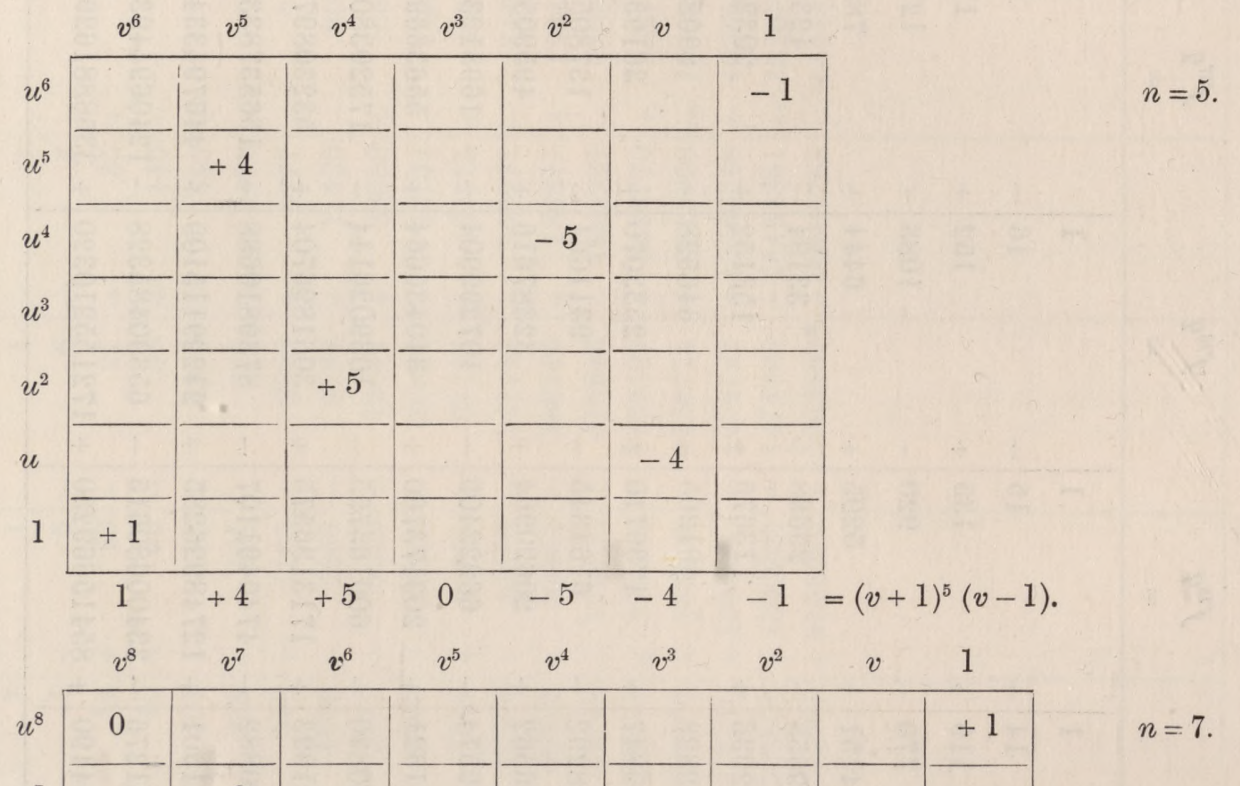

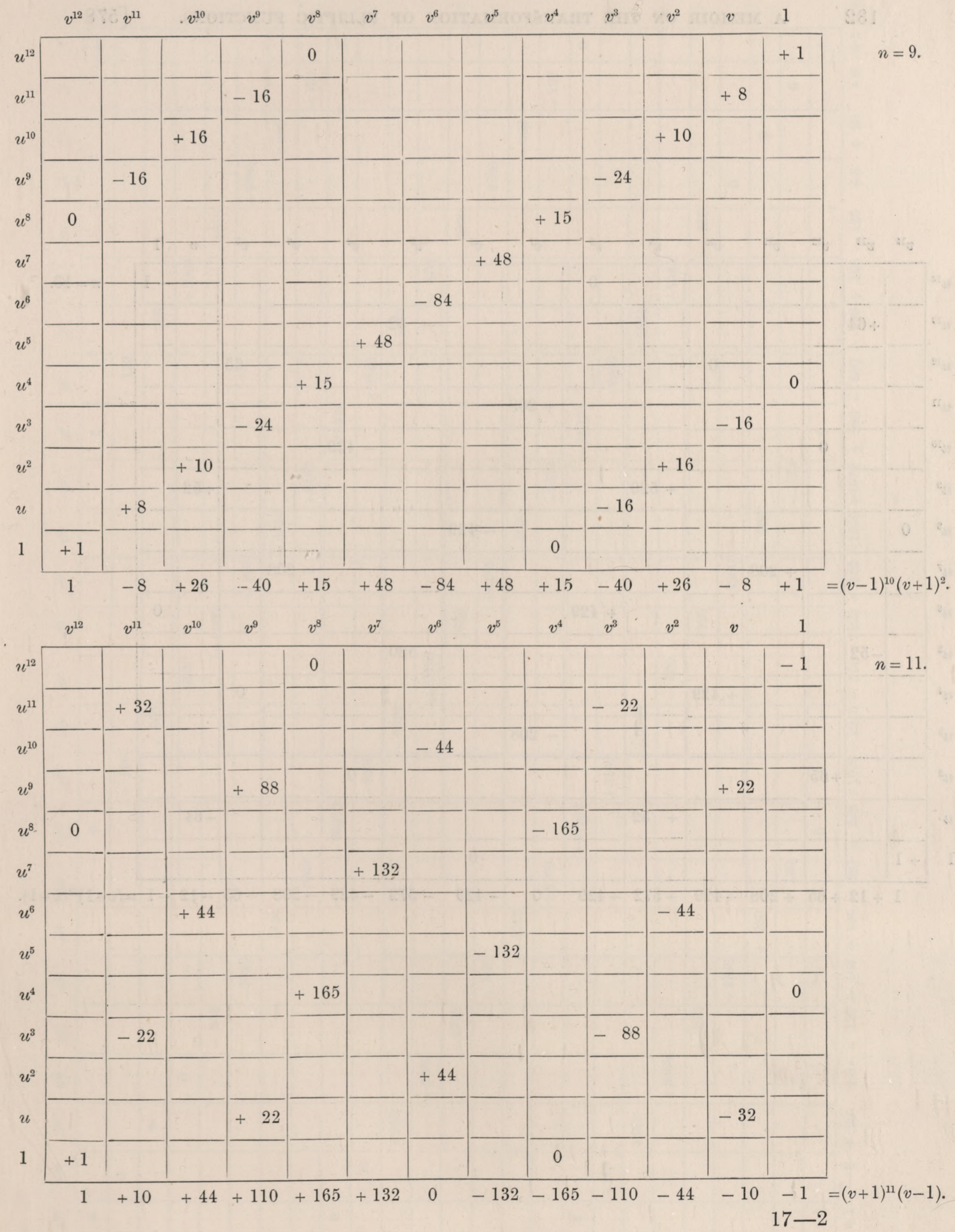


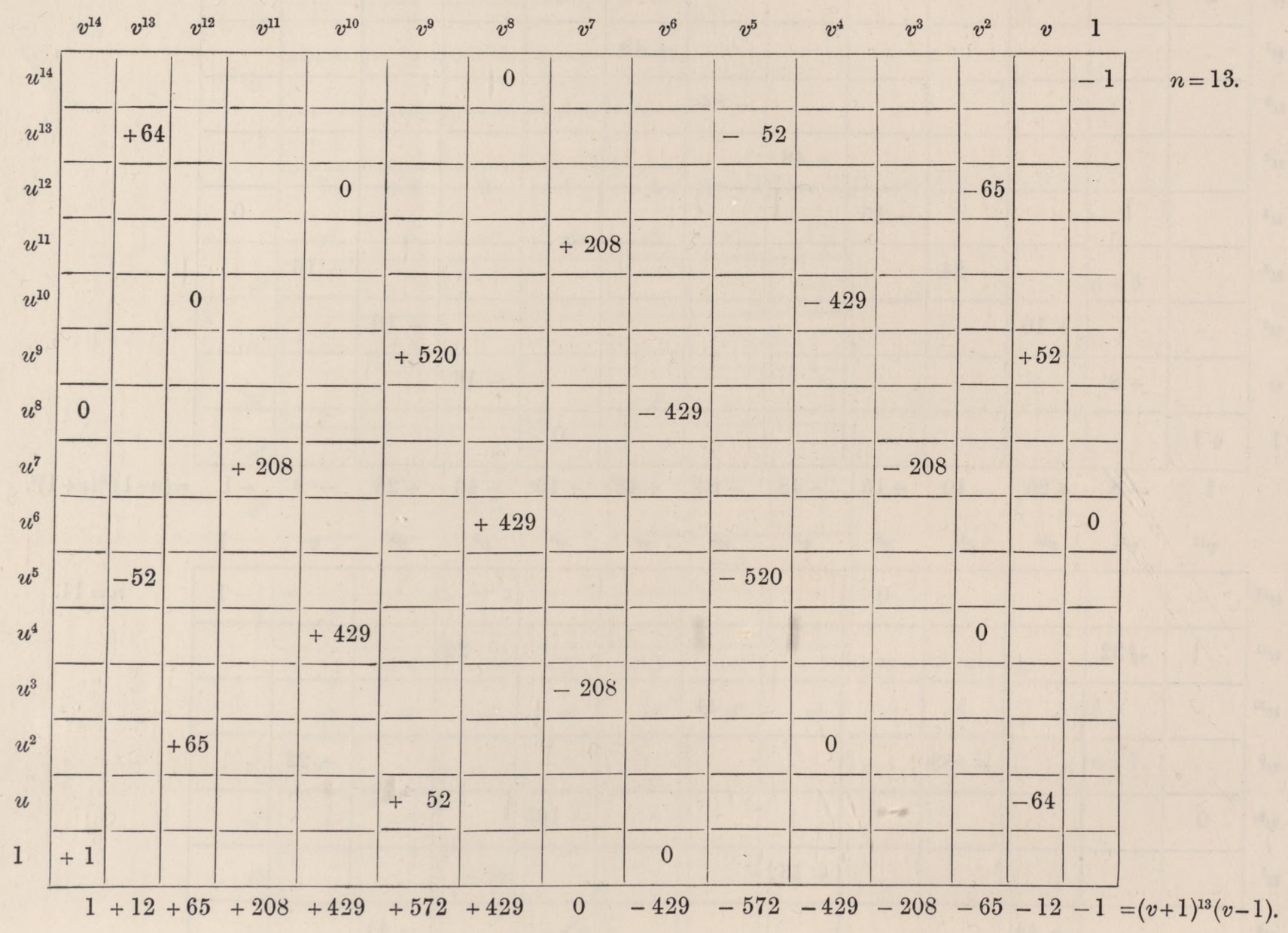




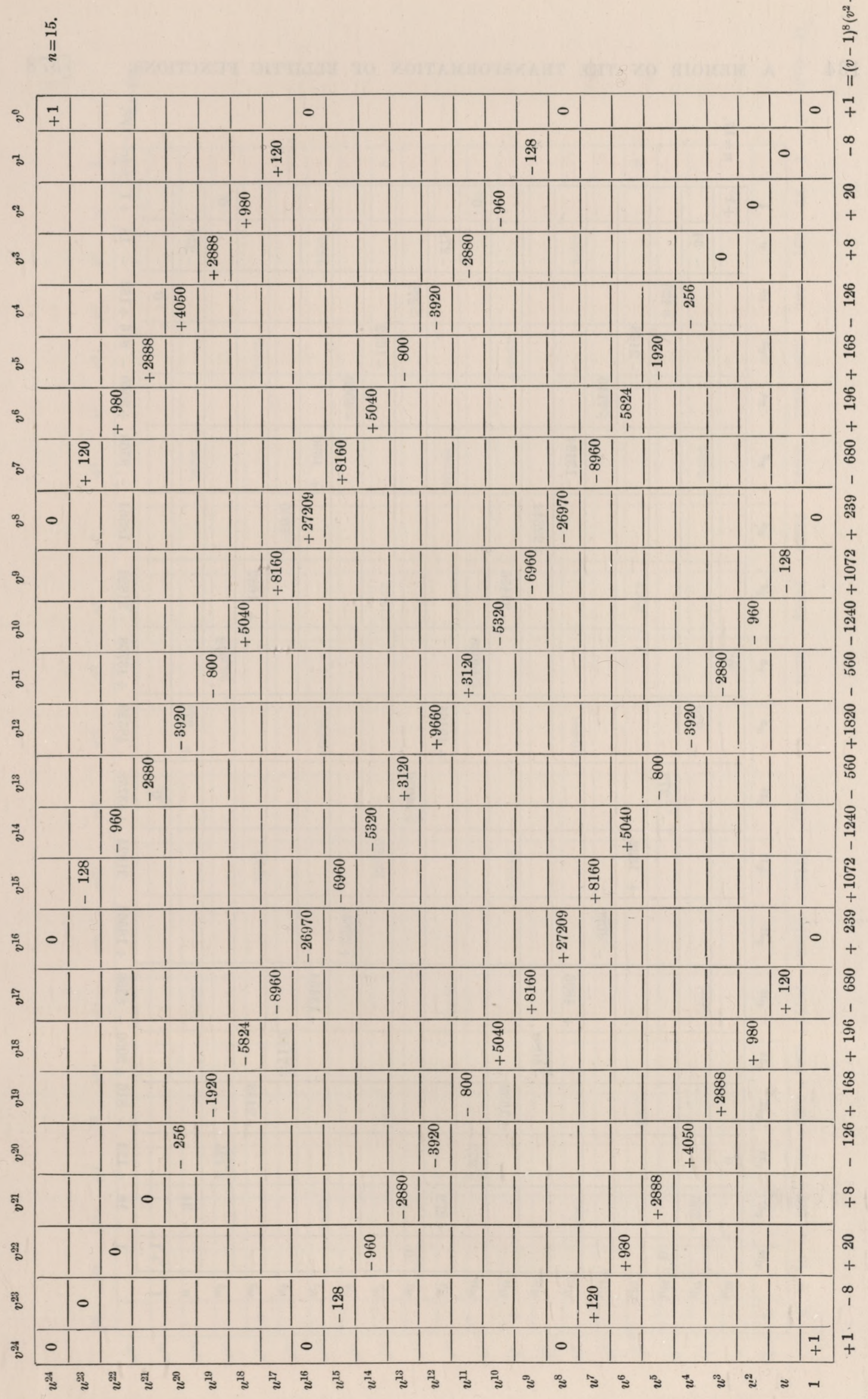


A MEMOIR ON THE TRANSFORMATION OF ELLIPTIC FUNCTIONS.

$[578$

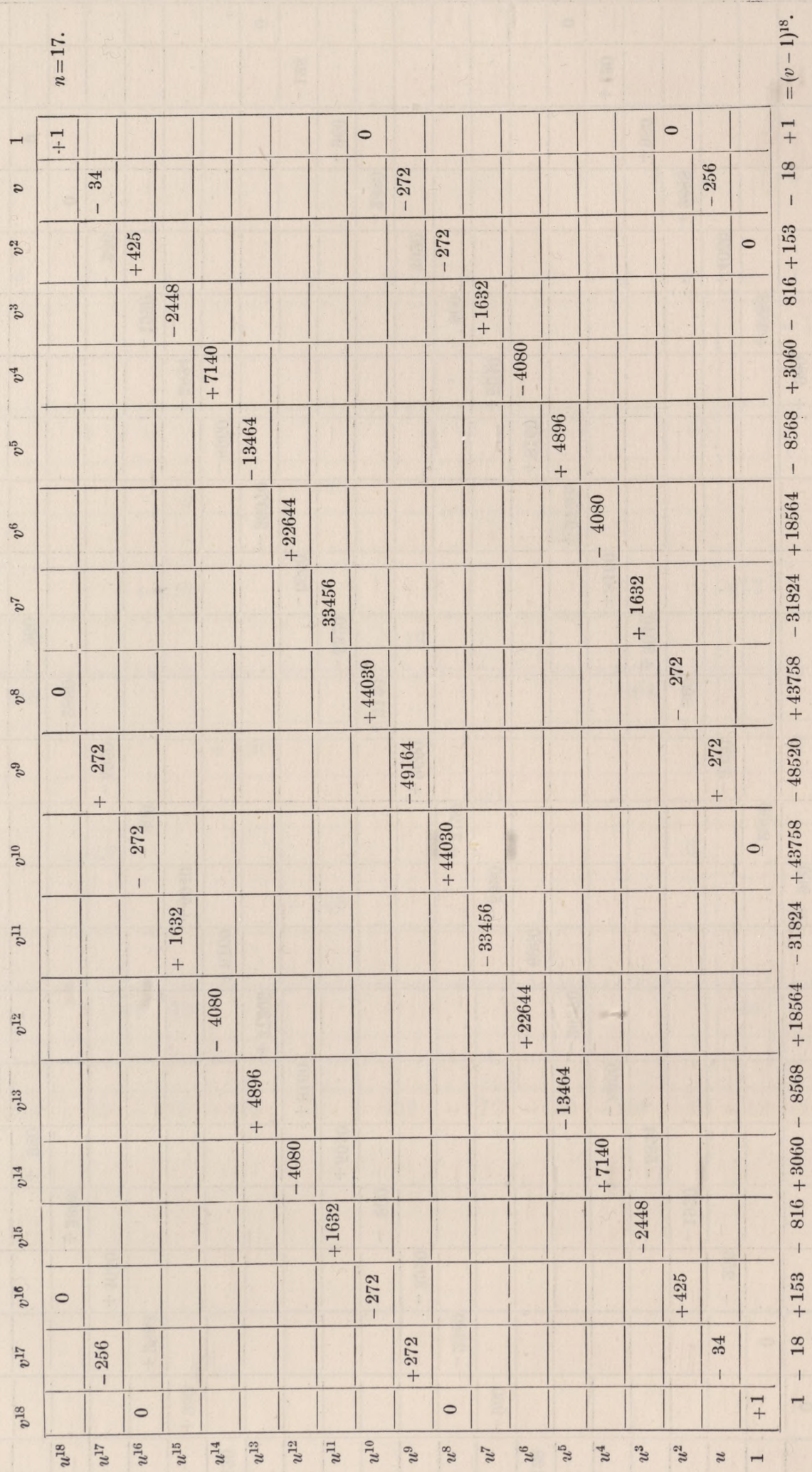




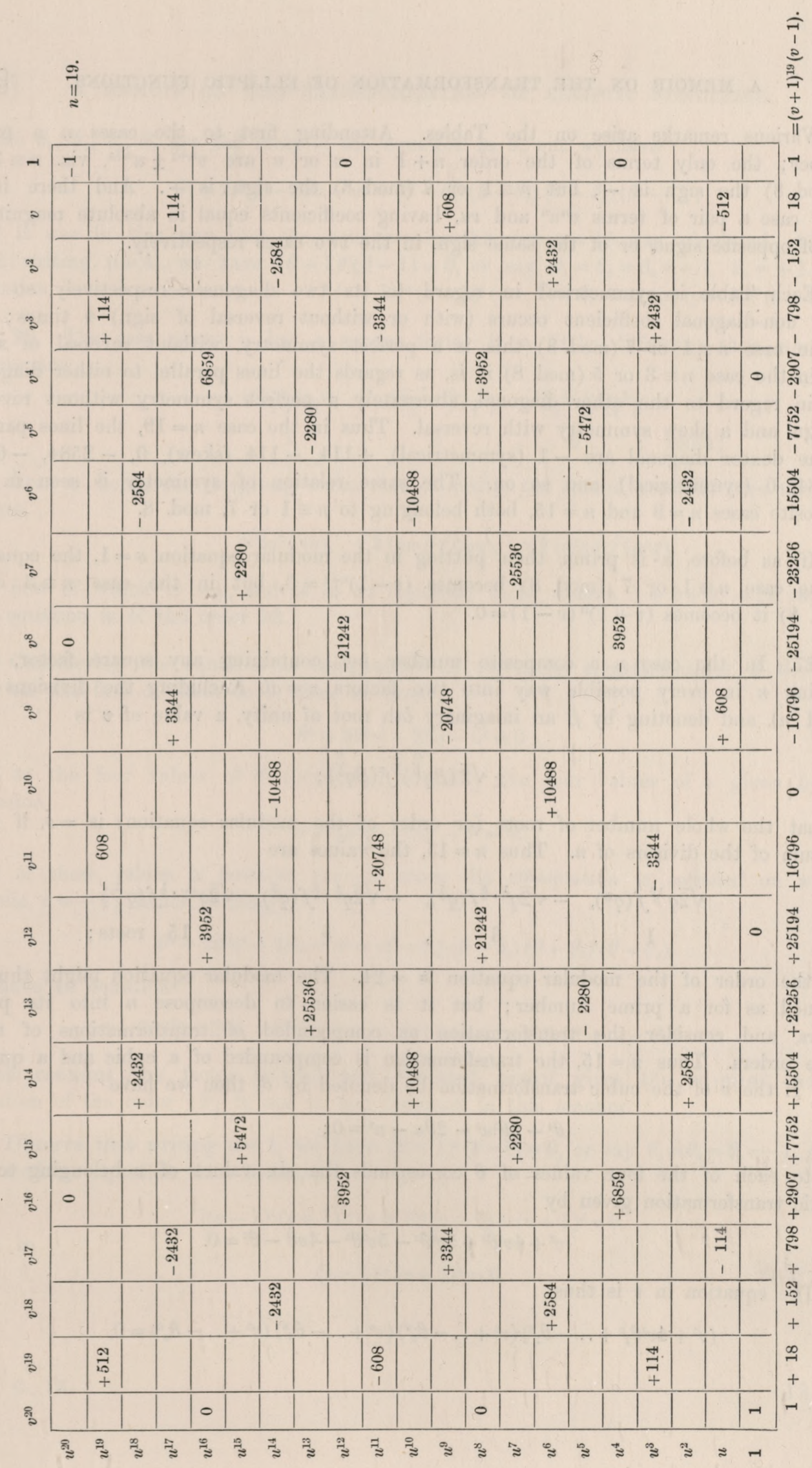


Various remarks arise on the Tables. Attending first to the cases $n$ a prime number; the only terms of the order $n+1$ in $v$ or $u$ are $v^{n+1} \pm u^{n+1}$, viz $n \equiv 3$ or $5(\bmod .8)$ the sign is - , but $n \equiv 1$ or $7(\bmod .8)$ the sign is + . And there is in every case a pair of terms $v^{n} u^{n}$ and $v u$, having coefficients equal in absolute magnitude, but of opposite signs, or of the same sign, in the two cases respectively.

Each Table is symmetrical in regard to its two diagonals respectively, so that every non-diagonal coefficient occurs (with or without reversal of sign) 4 times; viz. in the case $n \equiv 1$ or 7 (mod. 8 ) this is a perfect symmetry, without reversal of sign; but in the case $n \equiv 3$ or 5 (mod. 8 ) it is, as regards the lines parallel to either diagonal, and in regard to the other diagonal, alternately a perfect symmetry without reversal of sign and a skew symmetry with reversal. Thus in the case $n=19$, the lines parallel to the dexter diagonal are -1 (symmetrical), +114, - 114 (skew), 0, $-2584,-6859$, $-2584,0$ (symmetrical), and so on. The same relation of symmetry is seen in the composite cases $n=9$ and $n=15$, both belonging to $n \equiv 1$ or 7 , mod. 8 .

If, as before, $n$ is prime, then putting in the modular equation $u=1$, the equation in the case $n \equiv 1$ or 7 (mod. 8 ) becomes $(v-1)^{n+1}=0$, but in the case $n \equiv 3$ or 5 $(\bmod .8)$ it becomes $(v+1)^{n}(v-1)=0$.

27. In the case $n$ a composite number not containing any square factor, then dividing $n$ in every possible way into two factors $n=a b$ (including the divisions $n .1$ and $1 . n$ ), and denoting by $\beta$ an imaginary $b$ th root of unity, a value of $v$ is

$$
\pm \sqrt{2}\left(\beta q^{\frac{a}{b}}\right)^{\frac{1}{8}} f\left(\beta q^{\frac{a}{b}}\right)
$$

so that the whole number of roots (or order of the modular equation) is $=\nu$, if $\nu$ be the sum of the divisors of $n$. Thus $n=15$, the vaiues are

$$
\begin{array}{ccccc}
\sqrt{2} q^{\frac{15}{8}} f\left(q^{15}\right), & -\sqrt{2} q^{\frac{1}{8} \cdot \frac{5}{3}} f\left(q^{\frac{5}{3}}\right), & -\sqrt{2} q^{\frac{1}{8} \cdot \frac{3}{5}} f\left(q^{\frac{3}{5}}\right), & \sqrt{2} q^{\frac{1}{15} \cdot \frac{1}{8}} f\left(q^{\frac{1}{15}}\right) \\
1 & 3 & 3 & 5 & 15 \text { roots }
\end{array}
$$

and the order of the modular equation is $=24$. The modular equation might thus be obtained as for a prime number; but it is easier to decompose $n$ into its prime factors, and consider the transformation as compounded of transformations of these prime orders. Thus $n=15$, the transformation is compounded of a cubic and a quintic one. If the $v$ of the cubic transformation be denoted by $\theta$, then we have

$$
\theta^{4}+2 \theta^{3} u^{3}-2 \theta u-u^{4}=0
$$

and to each of the four values of $\theta$ corresponds the six values of $v$ belonging to the quintic transformation given by

$$
v^{6}+4 v^{5} \theta^{5}+5 v^{4} \theta^{2}-5 v^{2} \theta^{4}-4 v \theta-\theta^{6}=0 .
$$

The equation in $v$ is thus

$$
\left(v^{6}+4 v^{5} \theta_{1}{ }^{5}+\ldots-\theta_{1}{ }^{6}\right)\left(v^{6}+\ldots-\theta_{2}{ }^{6}\right)\left(v^{6}+\ldots-\theta_{3}{ }^{6}\right)\left(v^{6}+\ldots-\theta_{4}{ }^{6}\right)=0,
$$


where $\theta_{1}, \theta_{2}, \theta_{3}, \theta_{4}$ are the roots of the equation in $\theta$, viz. we have

$$
\theta^{4}+2 \theta^{3} u^{3}-2 \theta u-u^{4}=\left(\theta-\theta_{1}\right)\left(\theta-\theta_{2}\right)\left(\theta-\theta_{3}\right)\left(\theta-\theta_{4}\right) ;
$$

and it was in this way that the equation for the case $n=15$ was calculated. Observe that writing $u=1$, we have $(\theta+1)^{3}(\theta-1)=0$, or say $\theta_{1}=\theta_{2}=\theta_{3}=-1, \theta_{4}=+1$. The equation in $v$ thus becomes $\left\{(v-1)^{5}(v+1)\right\}^{3}(v+1)^{5}(v-1)=0$, that is, $(v-1)^{16}(v+1)^{8}=0$.

28. The case where $n$ has a square factor is a little different; thus $n=9$, the values are

$$
\begin{aligned}
& \sqrt{2} q^{\frac{9}{8}} f\left(q^{9}\right), \quad-\sqrt{2} q^{\frac{1}{8} \cdot \frac{3}{8}} f\left(q^{\frac{3}{8}}\right), \quad \sqrt{2} q^{\frac{1}{8} \cdot \frac{1}{9}} f\left(q^{\frac{1}{3}}\right), \\
& 1, \quad 3 \quad, \quad 9 \quad \text {, roots; }
\end{aligned}
$$

but here $\omega$ being an imaginary cube root of unity, the second term denotes the three values,

$$
\sqrt{2} q^{\frac{1}{8}} f(q), \quad \sqrt{2}(q \omega)^{\frac{1}{8}} f(\omega q), \quad \sqrt{2}\left(\omega^{2} q\right)^{\frac{1}{8}} f\left(\omega^{2} q\right),
$$

the first of which is $=u$, and is to be rejected; there remain $1+2+9,=12$ roots, or the equation is of the order 12.

Considering the equation as compounded of two cubic transformations, if the value of $v$ for the first of these be $\theta$, then we have

$$
\theta^{4}+2 \theta^{3} u^{3}-2 \theta u-u^{4}=0
$$

and to the four values of $\theta$ correspond severally the four values of $v$ given by the equation

$$
v^{4}+2 v^{3} \theta^{3}-2 v \theta-\theta^{4}=0 .
$$

One of these values is however $v=-u$, since the $v \theta$-equation is satisfied on writing therein $v=-u$; hence, writing

$$
\theta^{4}+2 \theta^{3} u^{3}-2 \theta u-u^{4}=\left(\theta-\theta_{1}\right)\left(\theta-\theta_{2}\right)\left(\theta-\theta_{3}\right)\left(\theta-\theta_{4}\right),
$$

we have an equation

$$
\left(v^{4}+2 v^{3} \theta_{1}^{3}-2 v \theta_{1}-\theta_{1}^{4}\right)\left(v^{4}+\ldots-\theta_{2}^{4}\right)\left(v^{4}+\ldots-\theta_{3}^{4}\right)\left(v^{4}+\ldots-\theta_{4}^{4}\right)=0,
$$

which contains the factor $(v+u)^{4}$ and, divested hereof, gives the required modular equation of the order 12 ; it was in fact obtained in this manner.

Observe that writing $u=1$, we have $(\theta+1)^{3}(\theta-1)=0$, or say $\theta_{1}=\theta_{2}=\theta_{3}=-1, \theta_{4}=1$; the modular equation then becomes

that is,

$$
\left\{(v-1)^{3}(v+1)\right\}^{3}(v+1)^{3}(v-1) \div(v+1)^{4}=0,
$$

$$
(v-1)^{10}(v+1)^{2}=0 .
$$

C. IX. 
The Multiplier Equation. Art. No. 29.

29. The theory is in many respects analogous to that of the modular equation. To each value of $v$ there corresponds a single value of $M$; hence $M$, or what is the same thing $\frac{1}{M}$, is determined by an equation of the same order as $v$, viz. $n$ being prime, the order is $=n+1$. The last term of the equation is constant, and the other coefficients are rational and integral functions of $u^{8}$, of a degree not exceeding $\frac{1}{2}(n-1)$; and not only so, but they are, $n \equiv 1$ (mod.4), rational and integral functions of $u^{8}\left(1-u^{8}\right)$, and $n \equiv 3$ (mod. 4), alternately of this form and of the same form multiplied by the factor $\left(1-2 u^{8}\right)$.

The values are in fact given as transcendental functions of $q$; viz. denoting by $M_{0}, M_{1}, M_{2}, \ldots, M_{n}$ the values corresponding to $v_{0}, v_{1}, v_{2}, \ldots, v_{n}$ respectively, and writing

then we have

$$
\begin{aligned}
\phi(q) & =\frac{(1+q)\left(1+q^{3}\right)\left(1+q^{5}\right) \ldots\left(1-q^{2}\right)\left(1-q^{4}\right)\left(1-q^{6}\right) \ldots}{(1-q)\left(1-q^{3}\right)\left(1-q^{5}\right) \ldots\left(1+q^{2}\right)\left(1+q^{4}\right)\left(1+q^{6}\right) \ldots} \\
& =1+2 q+2 q^{4}+2 q^{9}+2 q^{16}+\ldots
\end{aligned}
$$

$$
\begin{aligned}
& M_{0}=\frac{(-)^{\frac{n-1}{2}}}{\frac{\phi^{2}(q)}{\phi^{2}\left(q^{n}\right)}} \\
& M_{1}=\frac{\phi^{2}(q)}{\phi^{2}\left(\alpha q^{n}\right)}, \ldots(\alpha \text { an imaginary } n \text {th root of unity }) \\
& \vdots \\
& M_{n}=\frac{\phi^{2} q}{\phi^{2}\left(q^{\frac{1}{n}}\right)} .
\end{aligned}
$$

Hence, the form of the equation being known, the values of the numerical coefficients may be calculated; and it was in this way that Joubert obtained the following results. I have in some cases changed the sign of Joubert's multiplier, so that in every case the value corresponding to $u=0$ shall be $M=1$.

The equations are:

$$
\begin{array}{rlr}
n=3, & \frac{1}{M^{4}} & u=0, \text { this is } \\
+\frac{1}{M^{3}} \cdot 0 & \left(\frac{1}{M}-1\right)^{3}\left(\frac{1}{M}+3\right)=0 \\
+\frac{1}{M^{2}} \cdot-6 & u=1, \text { it is } \\
+\frac{1}{M} \cdot 8\left(1-2 u^{8}\right) & \left(\frac{1}{M}+1\right)^{3}\left(\frac{1}{M}-3\right)=0 . \\
-3=0 . &
\end{array}
$$




$$
\begin{aligned}
& n=5, \quad \frac{1}{M^{6}} \\
& +\frac{1}{M^{5}} \cdot-10 \\
& u=0 \text { or } 1 \text {, this is } \\
& +\frac{1}{M^{4}} \cdot+35 \\
& +\frac{1}{M^{3}} \cdot-60 \\
& +\frac{1}{M^{2}} \cdot+55 \\
& \frac{1}{M} \cdot-26+256 u^{8}\left(1-u^{8}\right) \\
& +5=0 . \\
& n=7, \quad \frac{1}{M^{8}} \\
& +\frac{1}{M^{7}} \cdot 0 \\
& +\frac{1}{M^{6}} \cdot-28 \\
& +\frac{1}{M^{5}}+112\left(1-2 u^{8}\right) \\
& \left(\frac{1}{M}-1\right)^{5}\left(\frac{1}{M}-5\right)=0 . \\
& +\frac{1}{M^{4}} \cdot-210 \\
& +\frac{1}{M^{3}} \cdot+224\left(1-2 u^{8}\right) \\
& +\frac{1}{M^{2}} \cdot-140-21.256 u^{8}\left(1-u^{8}\right) \\
& +\frac{1}{M} \cdot\left\{48+2048 u^{8}\left(1-u^{8}\right)\right\}\left(1-2 u^{8}\right) \\
& +7=0 \text {. } \\
& n=11, \quad \frac{1}{M^{12}} \\
& +\frac{1}{M^{11}} \cdot 0 \\
& u=0 \text {, this is } \\
& +\frac{1}{M^{10}} \cdot-66 \\
& \left(\frac{1}{M}-1\right)^{11}\left(\frac{1}{M}+11\right)=0 \text {; } \\
& +\frac{1}{M^{9}} \cdot+440\left(1-2 u^{8}\right) \\
& u=0 \text {, this is } \\
& \left(\frac{1}{M}-1\right)^{7}\left(\frac{1}{M}-7\right)=0 \text {; } \\
& u=1 \text {, it is } \\
& \left(\frac{1}{M}+1\right)^{7}\left(\frac{1}{M}+7\right)=0 \\
& u=1 \text {, it is } \\
& \left(\frac{1}{M}+1\right)^{11}\left(\frac{1}{M}-11\right)=0 .
\end{aligned}
$$




$$
\begin{aligned}
& +\frac{1}{M^{8}} \cdot-1485 \\
& +\frac{1}{M^{7}} \cdot+3168\left(1-2 u^{8}\right) \\
& +\frac{1}{M^{6}} \cdot-4620-3.11^{2} \cdot 256 u^{8}\left(1-u^{8}\right) \\
& +\frac{1}{M^{5}} \cdot\left\{+4752+11.4096 u^{8}\left(1-u^{8}\right)\right\}\left(1-2 u^{8}\right) \\
& +\frac{1}{M^{4}} \cdot-3465-3.7 .11 .512 u^{8}\left(1-u^{8}\right) \\
& +\frac{1}{M^{3}} \cdot\left\{+1760+11.83 .2048 u^{8}\left(1-u^{8}\right)\right\}\left(1-2 u^{8}\right) \\
& +\frac{1}{M^{2}} \cdot-594-9.11 .37 .256 u^{8}\left(1-u^{8}\right)-3.11 .131072\left\{u^{8}\left(1-u^{8}\right)\right\}^{2} \\
& +\frac{1}{M}\left\{120+15.4096 u^{8}\left(1-u^{8}\right)-524288\left\{u^{8}\left(1-u^{8}\right)\right\}^{8}\right\}\left(1-2 u^{8}\right) \\
& \quad-11=0 .
\end{aligned}
$$

The Multiplier as a rational function of $u, v$. Art. Nos. 30 to 36 .

30. The multiplier $M$, as having a single value corresponding to each value of $v$, is necessarily a rational function of $u, v$; and such an expression of $M$ can, as remarked by Königsberger, be deduced from the multiplier equation by means of Jacobi's theorem,

$$
M^{2}=\frac{1}{n} \frac{\lambda\left(1-\lambda^{2}\right)}{k\left(1-k^{2}\right)} \frac{d k}{d \lambda}
$$

viz. substituting for $k, \lambda$ their values $u^{8}, v^{8}$, and observing that if the modular equation be $F(u, v)=0$ so that the value of $\frac{d u}{d v}$ is $=-F^{\prime}(v) \div F^{\prime}(u)$, this is

$$
M^{2}=-\frac{1}{n} \frac{\left(1-v^{8}\right) v F^{\prime \prime} v}{\left(1-u^{8}\right) u F^{\prime \prime} u}
$$

and then in the multiplier equation separating the terms which contain the odd and even powers, and writing it in the form $\Phi\left(M^{2}\right)+M \Psi\left(M^{2}\right)=0$, this equation, substituting therein for $M^{2}$ its value, gives the value of $M$ rationally.

The rational expression of $M$ in terms of $u, v$ is of course indeterminate, since its form may be modified in any manner by means of the equation $F(u, v)=0$; and in the expression obtained as above, the orders of the numerator and the denominator are far too high. A different form may be obtained as follows: for greater convenience I seek for the value not of $M$ but of $\frac{1}{M}$. 
31. Denoting, as above, by $M_{0}, M_{1}, \ldots, M_{n}$ the values which correspond to $v_{0}, v_{1}, \ldots, v_{n}$ respectively, and writing $S \frac{1}{M}=\frac{1}{M_{0}}+\frac{1}{M_{1}}+\ldots+\frac{1}{M_{n}}$, \&c., we have $S \frac{1}{M}, S \frac{v}{M}$, \&c., all of them expressible as determinate functions of $u$; and we have moreover the theorem that each of these is a rational and integral function of $u$ : we have thus the series of equations

$$
S \frac{1}{M}=A, \quad S \frac{v}{M}=B, \ldots, S \frac{v^{n}}{M}=H,
$$

where $A, B, \ldots, H$ are rational and integral functions of $u$. These give linearly the different values of $\frac{1}{M}$; in fact, we have

$$
\left(v_{0}-v_{1}\right) \ldots\left(v_{0}-v_{n}\right) \frac{1}{M_{0}}=H-G S v_{1}+F S v_{1} v_{2}-\ldots \pm A v_{1} v_{2} \ldots v_{n},
$$

where $S v_{1}, S v_{1} v_{2}, \& c$. denote the combinations formed with the roots $v_{1}, v_{2}, \ldots, v_{n}$ (these can be expressed in terms of the single root $\left.v_{0}\right)$; and we have also $\left(v_{0}-v_{1}\right) \ldots\left(v_{0}-v_{n}\right)=F^{\prime}\left(v_{0}\right)$ : the resulting equation is consequently $F^{\prime} v_{0} \frac{1}{M_{0}}=R\left(u, v_{0}\right), R$ a determinate rational and integral function of $\left(u, v_{0}\right)$; but as the same formula exists for each root of the modular equation, we may herein write $M, v$ in place of $M_{0}, v_{0}$; and the formula thus is

$$
F^{\prime} v \cdot \frac{1}{M}=R(u, v)
$$

viz. we thus obtain the required value of $\frac{1}{M}$ as a rational traction, the denominator being the determinate function $F^{\prime} v$, and the numerator being, as is easy to see, a determinate function of the order $n$ as regards $v$.

32. The method is applicable when $M$ is only known by its expression in terms of $q$; but if we know for $M$ an expression in terms of $v, u$, then the method transforms this into a standard form as above. By way of illustration I will consider the case $n=3$, where the modular equation is

$$
v^{4}+2 v^{3} u^{3}-2 v u-u^{4}=0,
$$

and where a known expression of $M$ is $\frac{1}{M}=1+\frac{2 u^{3}}{v}$. Here writing $S_{-1}, S_{0}(=4), S$, \&c. to denote the sum of the powers $-1,0,1$, \&c. of the roots of the equation. we have

$$
\begin{aligned}
& S \frac{1}{M}=S_{0}+2 u^{3} S_{-1},=0, \text { as appears from the values presently given, } \\
& S \frac{v}{M}=S_{1}+2 u^{3} S_{0},=6 u^{3} \\
& S \frac{v^{2}}{M}=S_{2}+2 u^{3} S_{1},=0 \\
& S \frac{v^{3}}{M}=S_{3}+2 u^{3} S_{2},=6 u
\end{aligned}
$$


and observing that $v_{0}$ being ultimately replaced by $v$, we have

that is,

$$
S v_{1}=S v_{0}-v, \quad S v_{0} v_{1}=S v_{0} v_{1}-v S v_{0}+v^{2}, v_{1} v_{2} v_{3}=S v_{0} v_{1} v_{2}-v S v_{0} v_{1}+v_{2} S v_{0}-v^{2}
$$

$$
S v_{1}=-2 u^{3}-v, \quad S v_{1} v_{2}=2 u^{3} v+v^{2}, v_{1} v_{2} v_{3}=2 u-2 u^{3} v^{2}-v^{3}
$$

we have

viz. this is

$$
\begin{aligned}
F^{\prime} v \cdot \frac{1}{M}= & \left(S_{3}+2 u^{3} S_{2}\right) \\
& +\left(2 u^{3}+v\right)\left(S_{2}+2 u^{3} S_{1}\right) \\
& +\left(2 u^{3} v+v^{2}\right)\left(S_{1}+2 u^{3} S_{0}\right) \\
& +\left(-2 u+2 u^{3} v^{2}+v^{3}\right)\left(S_{0}+2 u^{3} S_{-1}\right)
\end{aligned}
$$

$$
\begin{aligned}
2\left(2 v^{3}+3 v^{2} u^{3}-u\right) \frac{1}{M}= & v^{3}\left(S_{0}+2 u^{3} S_{-1}\right) \\
& +v^{2}\left(S_{1}+4 u^{3} S_{0}+4 u^{6} S_{-1}\right) \\
& +v\left(S_{2}+4 u^{3} S_{1}+4 u^{6} S_{0}\right) \\
& +\left(S_{3}+4 u^{3} S_{2}+4 u^{6} S_{1}-2 u S_{0}-4 u^{4} S_{-1}\right)
\end{aligned}
$$

But we have

$$
S_{-1}=-\frac{2}{u^{3}}, \quad S_{0}=4, \quad S_{1}=-2 u^{3}, \quad S_{2}=4 u^{6}, \quad S_{3}=6 u-8 u^{9} ;
$$

and the equation thus is

$$
\left(2 v^{3}+3 v^{2} u^{3}-u\right) \frac{1}{M}=3\left(v^{2} u^{2}+2 u^{5} v+1\right) u
$$

to verify which observe that, substituting herein for $\frac{1}{M}$ its value $1+\frac{2 u^{3}}{v}$, the equation becomes

that is,

$$
\left(2 v^{3}+3 v^{2} u^{3}-u\right)\left(v+2 u^{3}\right)-3 v u\left(v^{2} u^{2}+2 u^{5} v+1\right)=0
$$

as it should do.

$$
2 v^{4}+4 v^{3} u^{3}-4 v u-2 u^{4}=0
$$

33. Any expression whatever of $M$ in terms of $u, v$ is in fact one of a system of four expressions; viz. we may simultaneously change

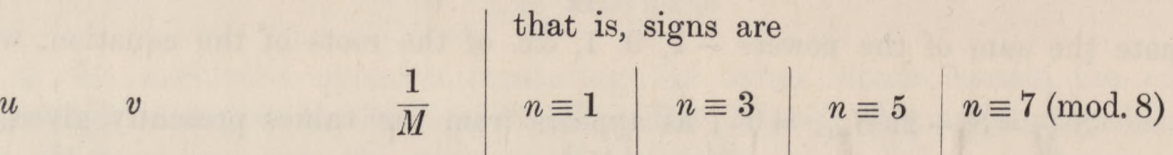

$$
\begin{aligned}
& \text { into } v,(-)^{\frac{n^{2}-1}{8}} u,(-)^{\frac{n-1}{2}} n M ;+++++-++ \\
& \text { or } \frac{1}{u}, \quad \frac{1}{v}, \quad \frac{v^{4}}{u^{4} M} ;+++++++++++++++ \\
& \text { or } \frac{1}{v},(-)^{\frac{n^{2}-1}{8}} \frac{1}{u},(-)^{\frac{n-1}{2}} \frac{u^{4}}{v^{4}} n M ;|+++|+-++++++
\end{aligned}
$$


Thus $n=3$, starting from $\frac{1}{M}=1+\frac{2 u^{3}}{v}$, we have

$$
\frac{1}{M}=1+\frac{2 u^{3}}{v}, \quad-3 M=1-\frac{2 v^{3}}{u}, \quad \frac{v^{4}}{u^{4} M}=1+\frac{2 v}{u^{3}}, \quad-\frac{u^{4}}{v^{4}} 3 M=1-\frac{2 u}{v^{3}} ;
$$

and of course if from any two of these we eliminate $M$, we have either an identity or the modular equation; thus we have the modular equation under the six different forms :

$$
\begin{array}{ll}
(1,2) & \left(v+2 u^{3}\right)\left(u-2 v^{3}\right)+3 u v=0, \\
(1,3) & v^{3}\left(v+2 u^{3}\right)-u\left(u^{3}+2 v\right)=0, \\
(1,4) \quad\left(v+2 u^{3}\right)\left(v^{3}-2 u\right)+3 u^{4}=0, \\
(2,3) \quad\left(u-2 v^{3}\right)\left(u^{3}+2 v\right)+3 v^{4}=0, \\
(2,4) \quad v\left(v^{3}-2 u\right)-u^{3}\left(u-2 v^{3}\right)=0, \\
(3,4) \quad\left(u^{3}+2 v\right)\left(v^{3}-2 u\right)+3 u^{3} v^{3}=0 .
\end{array}
$$

34. Next $n=5$. Here, starting from $\frac{1}{M}=\frac{v-u^{5}}{v\left(1-u v^{3}\right)}$, the changes give

$$
\frac{1}{M}=\frac{v-u^{5}}{v\left(1-u v^{3}\right)}, \quad 5 M=\frac{u+v^{5}}{u\left(1+u^{3} v\right)}, \quad \frac{v^{4}}{u^{4} M}=\frac{v^{3}\left(v-u^{5}\right)}{u^{4}\left(1-u v^{3}\right)}, \quad \frac{u^{4}}{v^{4}} 5 M=\frac{u^{3}\left(u+v^{5}\right)}{v^{4}\left(1+u^{3} v\right)},
$$

viz. the third and the fourth forms agree with the first and the second forms respectively; that is, there are only two independent forms, and the elimination of $M$ from these gives

$$
5 u v\left(1-u v^{3}\right)\left(1+u^{3} v\right)-\left(v-u^{5}\right)\left(u+v^{5}\right)=0,
$$

which is a form of the modular equation.

35. In the case $n=7$, starting from $\frac{1}{M}=\frac{-7 u(1-u v)\left(1-u v+u^{2} v^{2}\right)}{u-v^{7}}$ (as to this see post, No. 43), the forms are

$$
\begin{aligned}
\frac{1}{M} & =\frac{-7 u(1-u v)\left(1-u v+u^{2} v^{2}\right)}{u-v^{7}} . . \\
-7 M & =\frac{-7 v(1-u v)\left(1-u v+u^{2} v^{2}\right)}{v-u^{7}} \\
\frac{v^{4}}{u^{4} M} & =\frac{-7 v^{4}(1-u v)\left(1-u v+u^{2} v^{2}\right)}{u^{3}\left(u-v^{7}\right)} \\
-\frac{u^{4}}{v^{4}} 7 M & =\frac{-7 u^{4}(1-u v)\left(1-u v+u^{2} v^{2}\right)}{v^{3}\left(v-u^{7}\right)}
\end{aligned}
$$

so that here again the third and the fourth forms are identical with the second and the third forms respectively; there are thus only two forms, and the elimination of $M$ gives

$$
\left(u-v^{7}\right)\left(v-u^{7}\right)+7 u v(1-u v)^{2}\left(1-u v+u^{2} v^{2}\right)^{2}=0,
$$

which is a form of the modular equation. 
36. If in the foregoing equation

$$
F^{\prime} v \cdot \frac{1}{M}=R(u, v)
$$

we make the change $u, v, \frac{1}{M}$ into $v, \pm u, \pm n M$, it becomes

$$
\pm F^{\prime} u \cdot n M=R(v, \pm u) \text {; }
$$

combining these equations, we have

$$
\pm n M^{2} \cdot \frac{F^{\prime} u}{F^{\prime} v}=\frac{R(v, \pm u)}{R(u, v)}
$$

or, substituting herein the foregoing value

this becomes

$$
M^{2}=-\frac{1}{n} \frac{\left(1-v^{8}\right) v F^{\prime} v}{\left(1-u^{8}\right) u F^{\prime} u}
$$

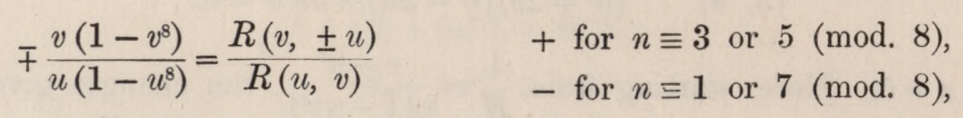

which must agree with the modular equation. Thus in the last-mentioned case $n=3$, we have

or, say

$$
\frac{1}{2} F^{\prime} v \cdot \frac{1}{M}=3\left(v^{2} u^{2}+2 u^{5} v+1\right) u,
$$

and therefore

the equation is

$$
R(u, v)=\left(v^{2} u^{2}+2 u^{5} v+1\right) u,
$$

$$
R(v,-u)=\left(v^{2} u^{2}-2 u v^{5}+1\right) v ;
$$

$$
+\frac{v\left(1-v^{8}\right)}{u\left(1-u^{8}\right)}=\frac{\left(v^{2} u^{2}-2 u v^{5}+1\right) v}{\left(v^{2} u^{2}+2 u^{5} v+1\right) u}
$$

which is right; because Jacobi, p. 82, [Ges. Werke, t. I., p. 137], for the modular equation, gives

$$
1-u^{8}=\left(1-u^{2} v^{2}\right)\left(v^{2} u^{2}+2 u^{5} v+1\right), \quad 1-v^{8}=\left(1-u^{2} v^{2}\right)\left(v^{2} u^{2}-2 u v^{5}+1\right) .
$$

Observe that the general equation

$$
\mp \frac{v\left(1-v^{8}\right)}{u\left(1-u^{8}\right)}=\frac{R(v, \pm u)}{R(u, v)}
$$

no longer contains the functions $F^{\prime} v, F^{\prime} u$, which enter into Jacobi's expression of $M^{2}$.

Theorem in connexion with the multiplication of Elliptic Functions. Art. Nos. 37 to 40.

37. The theory of multiplication gives an important theorem in regard to transformation. Starting with the $n$ thic transformation

$$
\frac{1-y}{1+y}=\frac{1-x}{1+x}\left(\frac{\alpha-\beta x+\gamma x^{2}-\ldots}{\alpha+\beta x+\gamma x^{2}+\ldots}\right)^{2},=\frac{1-x}{1+x}\left(\frac{P-Q x}{P+Q x}\right)^{2},
$$


we may form a like transformation,

$$
\frac{1-z}{1+z}=\frac{1-y}{1+y}\left(\frac{\alpha^{\prime}-\beta^{\prime} y+\gamma^{\prime} y^{2}-\ldots}{\alpha^{\prime}+\beta^{\prime} y+\gamma^{\prime} y^{2}+\ldots}\right)^{2}, \quad=\frac{1-y}{1+y}\left(\frac{P^{\prime}-Q^{\prime} y}{P^{\prime}+Q^{\prime} y}\right)^{2}
$$

such that the combination of the two gives a multiplication, viz. for the relation between $y, z$, deriving $w$ from $v$ as $v$ from $u$, we have $w=u$; and instead of $M$ we have $M^{\prime},= \pm \frac{1}{n M}$; that is, we have

and thence

$$
\begin{aligned}
& \frac{d x}{\sqrt{1-x^{2} \cdot 1-u^{8} x^{2}}}=\frac{M d y}{\sqrt{1-y^{2} \cdot 1-v^{8} y^{2}}}, \\
& \frac{d y}{\sqrt{1-y^{2} \cdot 1-v^{8} y^{2}}}=\frac{M^{\prime} d z}{\sqrt{1-z^{2} \cdot 1-u^{8} z^{2}}},
\end{aligned}
$$

$$
\frac{d x}{\sqrt{1-x^{2} \cdot 1-u^{8} x^{2}}}=\frac{ \pm \frac{1}{n} d z}{\sqrt{1-z^{2} \cdot 1-u^{8} z^{2}}}
$$

or, writing $x=\operatorname{sn} \theta$, we have $z= \pm \operatorname{sn} n \theta ; \pm$ is here $(-)^{\frac{n-1}{2}}$, viz. it is - for $n \equiv 3$ or 7 (mod. 8$)$, and + for $n \equiv 1$ or $5(\bmod .8)$.

Now in part effecting the substitution, we have

$$
\frac{1-z}{1+z}=\frac{1-x}{1+x}\left(\frac{P-Q x}{P+Q x}\right)^{2} \cdot\left(\frac{P^{\prime}-Q^{\prime} y}{P^{\prime}+Q^{\prime} y}\right)^{2}
$$

where $y$ denotes its value in terms of $x$.

And from the theory of elliptic functions, replacing $\operatorname{sn} n \theta, \operatorname{sn} \theta$ by their values $\pm z, x$, we have an equation

$$
\frac{1-z}{1+z}=\frac{1-x}{1+x}\left(\frac{A-B x+C x^{2}-\ldots}{A+B x+C x^{2}+\ldots}\right)^{2}
$$

where $A-B x+C x^{2}-\ldots, A+B x+C x^{2}+\ldots$ are given functions each of the order $\frac{1}{2}\left(n^{2}-1\right)$; viz. the coefficients are given functions of $k$, or, what is the same thing, of $u^{4}$. Comparing the two results, we see that in the $n$ thic transformation the sought-for function, $\alpha+\beta x+\gamma x^{2}+\ldots$ of the order $\frac{1}{2}(n-1)$, is a factor of a given function $A+B x+C x^{2}+\ldots$ of the order $\frac{1}{2}\left(n^{2}-1\right)$.

38. Considering the modular equation as known, then by what precedes we have

$$
\alpha+\beta x+\gamma x^{2}+\ldots=\alpha\left\{1+\frac{\beta}{\alpha} x+\ldots+\frac{u^{n}}{v} x^{\frac{1}{2}(n-1)}\right\}
$$

that is, the given function $A+B x+C x^{2}+\ldots$ has a factor $1+\frac{\beta}{\alpha} x+\ldots+\frac{u^{n}}{v} x^{\frac{1}{(n-1)}}$, of which one (the last) coefficient $\frac{u^{n}}{v}$ is known, and we are hence able theoretically to c. $1 \mathrm{x}$. 
determine all the other coefficients rationally in terms of $u, v$; that is, the modular equation being known, we can theoretically complete the solution of the transformation problem. I do not, however, see the way to obtaining a convenient solution in this manner.

39. The formula in question for $n=3$ is

$$
\frac{1+\operatorname{sn} 3 \theta}{1-\operatorname{sn} 3 \theta}=\frac{1-\operatorname{sn} \theta}{1+\operatorname{sn} \theta}\left(\frac{1+2 \operatorname{sn} \theta-2 k^{2} \operatorname{sn}^{3} \theta-k^{2} \operatorname{sn}^{4} \theta}{1-2 \operatorname{sn} \theta+2 k^{2} \operatorname{sn}^{3} \theta-k^{2} \operatorname{sn}^{4} \theta}\right)^{2}
$$

which, putting therein $x=\operatorname{sn} \theta, z=-\operatorname{sn} 3 \theta$, and replacing $k$ by $u^{4}$, may be written

$$
1+z(\div)=(1+x)\left(1-2 x+2 u^{8} x^{3}-u^{8} x^{4}\right)^{2}(\div)
$$

where the signs $(\div)$ indicate denominators which are obtained from the numerators by changing the signs of $z, x$ respectively.

The theorem in regard to $n=3$ thus is, $1+\frac{u^{3}}{v} x$ is a factor of $1-2 x+2 u^{8} x^{3}-u^{8} x^{4}$; viz. writing in the last-mentioned function $x=-\frac{v}{u^{3}}$, we ought to have

that is,

$$
0=1+2 \frac{v}{u^{3}}-2 \frac{v^{3}}{u}-\frac{v^{4}}{u^{4}}
$$

$$
u^{4}+2 u v-2 u^{3} v^{3}-v^{4}=0,
$$

which is in fact the modular equation.

40. And so for $n=5$, if $x=\operatorname{sn} \theta, z=\operatorname{sn} 5 \theta$; and for $n=7$, if $x=\operatorname{sn} \theta, z=-\operatorname{sn} 7 \theta$; the formulæ are:-

$$
n=5 \text {, }
$$

$$
n=7 \text {, }
$$

$$
\begin{aligned}
& 1+z=(1+x)\{\quad 1 \\
& (\div)+2 \quad x \\
& -4 \quad x^{2} \\
& 1+z=(1+x)\{\quad 1 \\
& (\div) \quad-4 \\
& -4 \\
& -10 u^{8} \quad x^{3} \\
& +4\left(2+7 u^{8}\right) \\
& +5 u^{8} \quad x^{4} \\
& -14 u^{8} \\
& +4 u^{8}\left(3+2 u^{8}\right) x^{5} \\
& -28 u^{8}\left(3+2 u^{8}\right) \\
& +4 u^{8}\left(1-u^{8}\right) x^{6} \\
& +28 u^{8}\left(4+u^{8}\right) \\
& +4 u^{8}\left(16+51 u^{8}+8 u^{16}\right) x^{7} \\
& -4 u^{8}\left(2+3 u^{8}\right) x^{7} \\
& -\quad u^{8}\left(144+305 u^{8}+16 u^{16}\right) x^{8} \\
& -5 u^{16} \quad x^{8} \\
& +10 u^{16} \quad x^{9} \\
& +4 u^{24} \quad x^{10} \\
& -2 u^{24} \quad x^{11} \\
& \left.-u^{24} \quad x^{12}\right\}^{2} \div \\
& -8 u^{8}\left(4+25 u^{8}+16 u^{16}\right) x^{9} \\
& +8 u^{8}\left(8+57 u^{8}+46 u^{16}\right) x^{10} \\
& +56 u^{18}\left(2+u^{8}\right) \quad x^{11} \\
& -4 u^{16}\left(56+161 u^{8}+56 u^{16}\right) x^{12}
\end{aligned}
$$


Term in \{\} has factor

$$
\begin{aligned}
& 1+\frac{\beta}{\alpha} x+\frac{u^{5}}{v} x^{2} \\
& \begin{aligned}
u=1, \text { term in }\{\} \text { is } \\
=(1+x)^{7}(1-x)^{5} .
\end{aligned}
\end{aligned}
$$

$$
\begin{aligned}
& +56 u^{24}\left(1+2 u^{8} \quad\right) x^{13} \\
& +8 u^{24}\left(46+57 u^{8}+8 u^{16}\right) x^{14} \\
& -8 u^{24}\left(16+25 u^{8}+4 u^{16}\right) x^{15} \\
& \text { - } u^{24}\left(16+305 u^{8}+144 u^{16}\right) x^{16} \\
& +4 u^{24}\left(8+51 u^{8}+16 u^{16}\right) x^{17} \\
& +28 u^{32}\left(1+4 u^{8}\right) \quad x^{18} \\
& -28 u^{32}\left(2+3 u^{8}\right) \quad x^{19} \\
& -14 u^{40} \quad x^{20} \\
& +4 u^{40}\left(7+2 u^{8}\right) \quad x^{21} \\
& -4 u^{43} \quad x^{22} \\
& -4 u^{48} \quad x^{23} \\
& \left.+u^{48} \quad x^{24}\right\}^{2}
\end{aligned}
$$

Term in \{\} has factor

$$
1+\frac{\beta}{\alpha} x+\frac{\gamma}{\alpha} x^{2}+\frac{u^{7}}{v} x^{3}
$$

$$
u=1 \text {, term in }\{\quad\} \text { is }(1+x)^{10}(1-x)^{14} \text {. }
$$

The transformations $n=3,5,7,11$. Art. Nos. 41 to 51 .

41. The cubic transformation, $n=3$.

I reproduce the results already obtained; since there are only two coefficients $\alpha, \beta$, these are also the last but one and last coefficients $\rho, \sigma$. Hence, from the values of $\alpha, \beta, \rho, \sigma$, we have

$$
\begin{aligned}
\alpha & =1, & 2 \alpha & =\frac{v^{3}}{u}\left(\frac{1}{M}-\frac{u^{4}}{v^{4}}\right), \\
2 \beta & =\frac{1}{M}-1, & \beta & =\frac{u^{3}}{v} ;
\end{aligned}
$$

the two values of $\frac{1}{M}$ are thus $\frac{1}{M}=2 \frac{u}{v^{3}}+\frac{u^{4}}{v^{4}},=1+\frac{2 u^{3}}{v}$, giving the modular equation

and we then have

$$
v^{4}+2 v^{3} u^{3}-2 v u-u^{4}=0 ;
$$

$$
\frac{1-y}{1+y}=\frac{1-x}{1+x}\left(\frac{v-u^{3} x}{v+u^{3} x}\right)^{2} .
$$


42. The quintic transformation, $n=5$.

Here there are the three coefficients $\alpha, \beta, \gamma$, or $\beta, \gamma$ are the last but one and last coefficients $\rho, \sigma$; we have

$$
\begin{aligned}
\alpha & =1, & 2 \beta & =v^{3} u\left(\frac{1}{M}-\frac{u^{4}}{v^{4}}\right), \\
2 \beta & =\frac{1}{M}-1, & \gamma & =\frac{u^{5}}{v} .
\end{aligned}
$$

Comparing the two values of $\beta$, we have $\frac{1}{M}=\frac{v-u^{5}}{v\left(1-v^{3} u\right)}$, and then

$$
\alpha=1, \quad 2 \beta=\frac{u\left(v^{4}-u^{4}\right)}{v\left(1-v^{3} u\right)}, \quad \gamma=\frac{u^{5}}{v}
$$

so that only the modular equation remains to be determined.

The unused equation is

$$
2 \alpha \gamma+2 \alpha \beta+\beta^{2}=\frac{v^{2}}{u^{2}}\left(2 \alpha \gamma+2 \beta \gamma+\beta^{2}\right)
$$

which, putting therein $\alpha=1$, may be written

$$
\left(2 \gamma+\beta^{2}\right)\left(u^{2}-v^{2}\right)=2 \beta\left(\gamma v^{2}-u^{2}\right)
$$

attending to the value of $\beta$, this divides by $u^{2}-v^{2}$; in fact the equation may be written

$$
2 \gamma+\beta^{2}=-\frac{u\left(v^{2}+u^{2}\right)}{v\left(1-v^{3} u\right)}\left(\gamma v^{2}-u^{2}\right)
$$

and then completing the substitution, and integralizing, this becomes

viz. this is

$$
\left\{8 v u^{3}\left(1-v^{3} u\right)^{2}+\left(v^{4}-u^{4}\right)^{2}\right\}=4 u v\left(u^{2}+v^{2}\right)\left(1-u^{3} v\right)\left(1-u v^{3}\right),
$$

$$
4\left(1-v^{3} u\right) u v\left\{2 u^{2}\left(1-v^{3} u\right)-\left(u^{2}+v^{2}\right)\left(1-v u^{3}\right)\right\}+\left(v^{4}-u^{4}\right)^{2}=0 ;
$$

and the term in \{\} being $=-\left(v^{2}-u^{2}\right)\left(1+v u^{3}\right)$, the whole again divides by $v^{2}-u^{2}$, and the equation thus becomes

$$
\left(v^{2}+u^{2}\right)\left(v^{4}-u^{4}\right)-4 u v\left(1-v^{3} u\right)\left(1+v u^{3}\right)=0,
$$

which is the modular equation.

43. The septic transformation, $n=7$.

I do not propose to complete the solution directly from the fundamental equations for $\alpha, \beta, \gamma, \delta$, but resort to the known modular equation, and to an expression of $M$ which I obtain by means thereof.

The modular equation is

$$
\left(1-u^{8}\right)\left(1-v^{8}\right)-(1-u v)^{8}=0
$$


which may also be written

$$
\left(v-u^{7}\right)\left(u-v^{7}\right)+7 u v(1-u v)^{2}\left(1-u v+u^{2} v^{2}\right)^{2}=0,
$$

as can be at once verified; but it also follows from Cauchy's identity

We then have

$$
(x+y)^{7}-x^{7}-y^{7}=7 x y(x+y)\left(x^{2}+x y+y^{2}\right)^{2} .
$$

Moreover

$$
M^{2}=-\frac{1}{n} \frac{\left(1-v^{8}\right) v F^{\prime} v}{\left(1-u^{8}\right) u F^{\prime} u}
$$

$$
\begin{aligned}
u F^{\prime} u & =-u^{8}\left(1-v^{8}\right)+u v(1-u v)^{7} \\
& =\frac{-u^{8}}{1-u^{8}}(1-u v)^{8}+u v(1-u v)^{7} \\
& =\frac{(1-u v)^{7}}{1-u^{8}} u\left(v-u^{7}\right) ;
\end{aligned}
$$

and similarly

$$
F^{\prime} v=\frac{(1-u v)^{7}}{1-v^{8}} v\left(u-v^{7}\right)
$$

whence

$$
\frac{1}{M^{2}}=\frac{-7 u}{v} \frac{\left(v-u^{7}\right)}{u-v^{7}}
$$

Writing this under the form

$$
\frac{1}{M^{2}}=\frac{-7 u v}{v^{2}} \frac{\left(v-u^{7}\right)\left(u-v^{7}\right)}{\left(u-v^{7}\right)^{2}},=\frac{49 u^{2}(1-u v)^{2}\left(1-u v+u^{2} v^{2}\right)^{2}}{\left(u-v^{7}\right)^{2}},
$$

I find, as will appear, that the root must be taken with the sign -, and that we thus have $\frac{1}{M}=-\frac{7 u(1-u v)\left(1-u v+u^{2} v^{2}\right)}{u-v^{7}}$, whence also $M=\frac{v(1-u v)\left(1-u v+u^{2} v^{2}\right)}{v-u^{7}}$.

44. Recurring now to the fundamental equations for the septic transformation, the coefficients are $\alpha, \beta, \gamma, \delta$, and we have

$$
\begin{aligned}
\alpha & =1, \\
2 \gamma & =u^{3} v^{3}\left(\frac{1}{M}-\frac{u^{4}}{v^{4}}\right), \\
2 \beta & =\frac{1}{M}-1, \quad \delta=\frac{u^{7}}{v},
\end{aligned}
$$

so that the coefficients are all given in terms of $v, M$. The unused equations are

$$
\begin{array}{ll}
u^{6}\left(2 \alpha \gamma+2 \alpha \beta+\beta^{2}\right) & =v^{2}\left(\gamma^{2}+2 \gamma \delta+2 \beta \delta\right), \\
u^{-2}\left(\gamma^{2}+2 \beta \gamma+2 \alpha \delta+2 \beta \delta\right) & =v^{2}\left(2 \alpha \gamma+2 \beta \gamma+2 \alpha \delta+\beta^{2}\right)
\end{array}
$$

which, substituting therein for $\alpha, \beta, \gamma, \delta$ the foregoing values, give two equations; from these, eliminating $M$, we should obtain the modular equation, and then $M$ in terms of $u, v$. 
Substituting in the first instance for $\alpha, \delta$ their values, the equations are

$$
\begin{gathered}
u^{6}\left(2 \beta+2 \gamma+\beta^{2}\right)=v^{2}\left\{\gamma^{2}+2 \frac{u^{7}}{v}(\beta+\gamma)\right\} \\
\gamma^{2}+2 \beta \gamma+(2+2 \beta) \frac{u^{7}}{v}=u^{2} v^{2}\left\{2 \gamma+2 \beta \gamma+2 \frac{u^{7}}{v}+\beta^{2}\right\} .
\end{gathered}
$$

The first of these is

$$
4(1-u v)(2 \beta+2 \gamma)+4 \beta^{2}-4 \frac{v^{2}}{u^{6}} \gamma^{2}=0
$$

viz. this is

$$
4(1-u v)\left(\frac{1}{M}-1+\frac{u^{3} v^{3}}{M}-\frac{u^{7}}{v}\right)+\left(\frac{1}{M}-1\right)^{2}-v^{8}\left(\frac{1}{M}-\frac{u^{4}}{v^{4}}\right)^{2}=0
$$

or observing that in this equation the coefficient of $\frac{2}{M}$ is

$$
\begin{aligned}
& \left(1-u^{2} v^{2}\right)\left\{2-2 u v+2 u^{2} v^{2}-1-u^{2} v^{2}\right\}, \\
= & \left(1-u^{2} v^{2}\right)(1-u v)^{2},=(1-u v)^{3}(1+u v),
\end{aligned}
$$

the equation becomes

$$
\left(1-v^{8}\right) \frac{1}{M^{2}}+\frac{2}{M}(1-u v)^{3}(1+u v)+1-u^{8}-4(1-u v)\left(1+\frac{u^{7}}{v}\right)=0
$$

45. This should be satisfied identically by the foregoing value of $\frac{1}{M}$; viz. it should be satisfied on writing therein

that is, we should have

$$
\begin{aligned}
& \frac{1}{M^{2}}=-\frac{7 u}{v} \frac{v-u^{7}}{u-v^{7}} \\
& \frac{1}{M}=-\frac{7 u(1-u v)\left(1-u v+u^{2} v^{2}\right)}{u-v^{7}}
\end{aligned}
$$

$$
\begin{aligned}
-7 \frac{u}{v}\left(v-u^{7}\right)\left(1-v^{8}\right) & -14 u(1-u v)^{4}\left(1+u^{3} v^{3}\right) \\
+ & \left(u-v^{7}\right)\left\{1-u^{8}-4(1-u v)\left(1+\frac{u^{7}}{v}\right)\right\}=0,
\end{aligned}
$$

where observe that the - sign of the second term is the sign of the foregoing value of $\frac{1}{M}$; so that the identity being verified, it follows that the correct sign has been attributed to the value of $\frac{1}{M}$.

46. Multiplying by $v$, the equation is

$$
\begin{aligned}
-7\left(1-u^{8}-\overline{1-u v}\right)\left(1-v^{8}\right)-14 u v(1-u v)^{4}\left(1+u^{3} v^{3}\right) \\
+\left\{1-v^{8}-\overline{1-u v}\right\}\left\{-8(1-u v)+1-u^{8}\right\}+4(1-u v)\left(v-u^{7}\right)\left(u-v^{7}\right)=0,
\end{aligned}
$$


viz. this is

$$
\begin{aligned}
-7\left(1-u^{8}\right)\left(1-v^{8}\right)+7(1-u v)\left(1-v^{8}\right)-14 u v & (1-u v)^{4}\left(1+u^{8} v^{3}\right) \\
+\quad\left(1-u^{8}\right)\left(1-v^{8}\right)-8(1-u v)\left(1-v^{8}\right)+8 & (1-u v)^{2} \\
-1(1-u v)\left(1-u^{8}\right)+4 & (1-u v)\left(v-u^{7}\right)\left(u-v^{7}\right)=0 .
\end{aligned}
$$

In the second column the coefficient of $1-u v$ is $2-u^{8}-v^{8}$, viz. this is

$$
=\left(1-u^{8}\right)\left(1-v^{8}\right)+1-(u v)^{8} \text {, or it is }=(1-u v)^{8}+1-(u v)^{8} .
$$

Reducing also the other two columns by means of the modular equation, the equation thus becomes

$$
\begin{aligned}
-6(1-u v)^{8}-(1-u v)\left\{(1-u v)^{8}+1-(u v)^{8}\right\} & -14 u v(1-u v)^{4}\left(1+u^{3} v^{3}\right) \\
& +8(1-u v)^{2} \\
& -28 u v(1-u v)^{3}\left(1-u v+u^{2} v^{2}\right)^{2}=0 .
\end{aligned}
$$

This is in fact an identity; to show it, writing for convenience $\theta$ in place of $u v$, and observing that the terms

are

$$
\begin{aligned}
& -(1-\theta)\left(1-\theta^{8}\right)+8(1-\theta)^{2}, \\
= & (1-\theta)^{2}\left\{8-\left(1+\theta+\theta^{2}+\theta^{3}+\theta^{4}+\theta^{5}+\theta^{6}+\theta^{7}\right)\right\}
\end{aligned}
$$

$$
=(1-\theta)^{3}\left(7+6 \theta+5 \theta^{2}+4 \theta^{3}+3 \theta^{4}+2 \theta^{5}+\theta^{6}\right),
$$

the whole equation divides by $(1-\theta)^{3}$; or throwing out this factor, it is .

$$
\begin{aligned}
-6(1-\theta)^{5}-(1-\theta)^{6}+7+6 \theta & +5 \theta^{2}+4 \theta^{3}+3 \theta^{4}+2 \theta^{5}+\theta^{6} \\
& -14 \theta(1-\theta)\left(1+\theta^{3}\right)-28 \theta\left(1-\theta+\theta^{2}\right)^{2}=0 .
\end{aligned}
$$

The first line is $=14 \theta\left(3-5 \theta+6 \theta^{2}-3 \theta^{3}+\theta^{4}\right)$; whence, throwing out the factor $14 \theta$, the equation is

that is,

$$
3-5 \theta+6 \theta^{2}-3 \theta^{3}+\theta^{4}-(1-\theta)\left(1+\theta^{3}\right)-2\left(1-\theta+\theta^{2}\right)^{2},
$$

$$
\left(1-\theta+\theta^{2}\right)\left(3-2 \theta+\theta^{2}\right)-\left(1-\theta^{2}\right)\left(1-\theta+\theta^{2}\right)-2\left(1-\theta+\theta^{2}\right)^{2}=0 ;
$$

or throwing out the factor $1-\theta+\theta^{2}$, the equation is

$$
\left(3-2 \theta+\theta^{2}\right)-\left(1-\theta^{2}\right)-2\left(1-\theta+\theta^{2}\right)=0,
$$

which is an identity.

The other equation is

that is,

$$
\gamma^{2}+2 \beta \gamma+(2+2 \beta) \frac{u^{7}}{v}=u^{2} v^{2}\left(2 \gamma+2 \beta \gamma+2 \frac{u^{7}}{v}+\beta^{2}\right)
$$

$$
\gamma^{2}+2 \beta \gamma-u^{2} v^{2} \beta^{2}+2(1+\beta)\left(\frac{u^{7}}{v}-\gamma u^{2} v^{2}\right)-2 u^{9} v=0,
$$

which might also be verified, but I have not done this. 
47. The conclusion is

$$
\alpha=1, \quad \beta=\frac{1}{2}\left(\frac{1}{M}-1\right), \quad \gamma=\frac{1}{2} u^{3} v^{3}\left(\frac{1}{M}-\frac{u^{4}}{v^{4}}\right), \quad \delta=\frac{u^{7}}{v},
$$

where

$$
\frac{1}{M}=\frac{-7 u(1-u v)\left(1-u v+u^{2} v^{2}\right)}{u-v^{7}}
$$

and of course

$$
\frac{1-y}{1+y}=\frac{1-x}{1+x}\left(\frac{1-\beta x+\gamma x^{2}-\delta x^{3}}{1+\beta x+\gamma x^{2}+\delta x^{3}}\right)^{2}
$$

but the resulting form may admit of simplification.

48. The endecadic transformation, $n=11$.

I have not completed the solution, but the results, so far as I have obtained them, are interesting. The coefficients are $\alpha, \beta, \gamma, \delta, \epsilon, \zeta$; and we have, as in general,

$$
\begin{aligned}
\alpha & =1 \quad, \quad 2 \epsilon=u^{7} v^{3}\left(\frac{1}{M}-\frac{u^{4}}{v^{4}}\right), \\
2 \beta & =\frac{1}{M}-1, \quad \zeta=\frac{u^{11}}{v} .
\end{aligned}
$$

The unused equations then are

$$
\begin{aligned}
u^{14}\left(2 \alpha \gamma+2 \alpha \beta+\beta^{2}\right) & =v^{2}\left(\epsilon^{2}+2 \epsilon \zeta+2 \delta \zeta\right), \\
u^{6}\left(\gamma^{2}+2 \alpha \epsilon+2 \alpha \delta+2 \beta \gamma+2 \beta \delta\right) & =v^{2}\left(2 \gamma \epsilon+2 \gamma \zeta+2 \delta \epsilon+2 \beta \zeta+\delta^{2}\right), \\
u^{-2}\left(2 \gamma \epsilon+2 \alpha \zeta+2 \gamma \delta+2 \beta \epsilon+2 \beta \zeta+\delta^{2}\right) & =v^{2}\left(\gamma^{2}+2 \alpha \epsilon+2 \alpha \zeta+2 \gamma \delta+2 \beta \epsilon+2 \beta \delta\right), \\
u^{-10}\left(\epsilon^{2}+2 \gamma \zeta+2 \delta \epsilon+2 \delta \zeta\right) & =v^{2}\left(2 \alpha \gamma+2 \alpha \delta+2 \beta \gamma+\beta^{2}\right) ;
\end{aligned}
$$

but I attend only to the first and the last, which, it will be observed, contain $\gamma, \delta$ linearly. If in the first instance we substitute only for $\alpha, \zeta$ their values, the equations become

$$
\begin{aligned}
& u^{12} \beta(2+\beta)-\frac{v^{2}}{u^{2}} \epsilon\left(\epsilon+2 \frac{u^{11}}{v}\right) \quad+u^{12} \cdot 2 \gamma \quad-v u^{9} \cdot 2 \delta=0, \\
& u^{-12} \epsilon^{2} \quad-\frac{v^{2}}{u^{2}} \beta^{2} \quad+\left\{\frac{1}{u v}-\frac{v^{2}}{u^{2}}(1+\beta)\right\}, 2 \gamma+\left\{\frac{1}{u v}-\frac{v^{2}}{u^{2}}+u^{-12} \epsilon\right\} \cdot 2 \delta=0 ;
\end{aligned}
$$

say, for a moment, these are

$$
\begin{aligned}
& A+P .2 \gamma+Q \cdot 2 \delta=0 \\
& B+R \cdot 2 \gamma+S .2 \delta=0
\end{aligned}
$$

giving

$$
1: 2 \gamma: 2 \delta=P S-Q R: Q B-S A: R A-P B .
$$

Here

$$
\begin{aligned}
P S-Q R & =\frac{u^{11}}{v}+\epsilon-u^{10} v^{2}+u^{8}-u^{7} v^{3}(1+\beta) \\
& =\frac{1}{2}\left\{\frac{2 u^{11}}{v}+\left(u^{7} v^{3} \frac{1}{M}-\frac{u^{11}}{v}\right)-2 u^{10} v^{2}+2 u^{8}-2 u^{7} v^{3}-\left(u^{7} v^{3} \frac{1}{M}-u^{7} v^{3}\right)\right\},
\end{aligned}
$$


where the terms containing $\frac{1}{M}$ disappear of themselves, viz. this is

$$
\begin{aligned}
& =\frac{1}{2}\left(\frac{u^{11}}{v}-2 u^{10} v^{2}+2 u^{8}-u^{7} v^{3}\right) \\
& =-\frac{1}{2} \frac{u^{7}}{v}\left(v^{4}+2 v^{3} u^{3}-2 v u-u^{4}\right) ;
\end{aligned}
$$

observe that the term in ( ), equated to zero, gives the modular equation for the case $n=3$. It thus appears that $\gamma$ and $\delta$ are given as fractions, having in their denominator this function $u^{4}+2 u v-2 u^{3} v^{3}-v^{4}$.

49. To complete the calculation, we have

$$
\begin{aligned}
Q B-A S= & -v u^{9}\left(\frac{\epsilon^{2}}{u^{12}}-\frac{v^{2}}{u^{2}} \beta^{2}\right) \\
& -\left\{u^{12} \beta(2+\beta)-\frac{v^{2}}{u^{2}} \epsilon\left(\epsilon+2 \frac{u^{11}}{v}\right)\right\}\left\{\frac{1}{u v}-\frac{v^{2}}{u^{2}}+\frac{\epsilon}{u^{12}}\right\} ;
\end{aligned}
$$

viz. multiplying by 8 , and substituting for $2 \beta, 2 \epsilon$ their values, this is

$$
\begin{aligned}
8(Q B-A S)= & -2 v u^{\theta}\left\{u^{2} v^{6}\left(\frac{1}{M}-\frac{u^{4}}{v^{4}}\right)^{2}-\frac{v^{2}}{u^{2}}\left(\frac{1}{M}-1\right)^{2}\right\} \\
& -\left\{u^{12}\left(\frac{1}{M}-1\right)\left(\frac{1}{M}+3\right)-u^{12} v^{8}\left(\frac{1}{M}-\frac{u^{4}}{v^{4}}\right)\left(\frac{1}{M}+\frac{3 u^{4}}{v^{4}}\right)\right\}\left(\frac{1}{u v}-\frac{2 v^{2}}{u^{2}}+\frac{v^{3}}{u^{5}} \frac{1}{M}\right),
\end{aligned}
$$

or, what is the same thing,

$$
\begin{aligned}
-\frac{8 v}{u^{7}}(Q B-A S) & =2\left\{u^{4} v^{8}\left(\frac{1}{M}-\frac{u^{4}}{v^{4}}\right)^{2}-v^{4}\left(\frac{1}{M}-1\right)^{2}\right\} \\
& +\left\{\left(\frac{1}{M}-1\right)\left(\frac{1}{M}+3\right)-v^{8}\left(\frac{1}{M}-\frac{u^{4}}{v^{4}}\right)\left(\frac{1}{M}+3 \frac{u^{4}}{v^{4}}\right)\right\}\left(u^{4}-2 v^{3} u^{3}+\frac{v^{4}}{M}\right),
\end{aligned}
$$

viz. the left-hand side is

$$
\begin{aligned}
& =2\left\{-v^{4}\left(1-u^{4} v^{4}\right) \frac{1}{M^{2}}+2 v^{4}\left(1-u^{8}\right) \frac{1}{M}+u^{12}-v^{4}\right\} \\
& +\left\{\frac{1}{M^{2}}\left(1-v^{8}\right)+\frac{2}{M}\left(1-u^{4} v^{4}\right)-3\left(1-u^{8}\right)\right\}\left\{\frac{1}{M}+u^{3}\left(u-2 v^{3}\right)\right\}
\end{aligned}
$$

or, say we have $-\frac{8 v}{u^{7}}(Q B-S A)=\Pi$, where

$$
\begin{aligned}
\Pi= & \frac{1}{M^{3}} \cdot v^{4}\left(1-v^{8}\right) \\
& +\frac{1}{M^{2}} \cdot u^{3}\left(u-2 v^{3}\right)\left(1-v^{8}\right) \\
& +\frac{1}{M} \cdot 4 u^{7} v^{7}+v^{4}\left(1-3 u^{8}\right)--4 u^{3} v^{3}+2 u^{4} \\
& +\quad \cdot-2 v^{4}+6 v^{3} u^{3}\left(1-u^{8}\right)+u^{4}\left(-3+5 u^{8}\right)
\end{aligned}
$$

C. IX. 
wherefore the value of $2 \gamma$ is $=\frac{1}{4} \Pi \div\left(v^{4}+2 v^{3} u^{3}-2 v u-u^{4}\right)$. Similarly, writing

$$
\begin{aligned}
\Pi^{\prime}= & \frac{1}{M^{3}} \cdot v^{4}\left(1-v^{8}\right) \\
& +\frac{1}{M^{2}} \cdot v\left(v^{3}-2 u\right)\left(1-v^{8}\right) \\
& +\frac{1}{M} \cdot 4 u^{5} v^{5}+v^{4}\left(3-u^{8}\right)+4 u v-2 u^{4} v^{8} \\
& +\quad v^{4}\left(-5+3 u^{8}\right)+6 v u\left(1-u^{8}\right)+2 u^{12}
\end{aligned}
$$

we find

$$
2 \delta=\frac{1}{4} \frac{u^{3}}{v} \Pi^{\prime} \div\left(v^{4}+2 v^{3} u^{3}-2 v u-u^{4}\right)
$$

in verification whereof observe that this being so, the first equation gives the identity

$$
\left\{\left(\frac{1}{M}-1\right)\left(\frac{1}{M}+3\right)-v^{8}\left(\frac{1}{M}-\frac{u^{4}}{v^{4}}\right)\left(\frac{1}{M}+\frac{3 u^{4}}{v^{4}}\right)\right\}\left(v^{4}+2 v^{3} u^{3}-2 v u-u^{4}\right)+\Pi-\Pi^{\prime}=0 .
$$

50. The result is that, writing for the moment $v^{4}+2 v^{3} u^{3}-2 v u-u^{4}=\Delta$, the values of the coefficients are

$$
\begin{aligned}
& \alpha, \beta, \gamma, \delta, \epsilon, \zeta, \\
& =1, \quad \frac{1}{2}\left(\frac{1}{M}-1\right), \quad \frac{1}{8} \frac{\Pi}{\Delta}, \quad \frac{1}{8} \frac{u^{3}}{v} \frac{\Pi^{\prime}}{\Delta}, \quad \frac{1}{2} u^{7} v^{3}\left(\frac{1}{M}-\frac{u^{4}}{v^{4}}\right), \quad \frac{u^{11}}{v},
\end{aligned}
$$

and

$$
\frac{1-y}{1+y}=\frac{1-x}{1+x}\left(\frac{1-\beta x+\gamma x^{2}-\delta x^{3}+\epsilon x^{4}-\zeta x^{5}}{1+\beta x+\gamma x^{2}+\delta x^{3}+\epsilon x^{4}+\zeta x^{5}}\right)^{2}
$$

the modular equation is known, and to complete the solution we require only an expression for $M$ in terms of $u, v$.

51. We may herein illustrate the following theorem, viz. we may simultaneously change $u, v, \frac{1}{M}, \alpha: \beta: \gamma: \delta: \epsilon: \zeta ;$ into $\frac{1}{u}, \frac{1}{v}, \frac{v^{4}}{u^{4}} \frac{1}{M}, \zeta: \epsilon: \delta: \gamma: \beta: \alpha$.

Thus making the change in the equation

$$
\frac{\beta}{\alpha}=\frac{1}{2}\left(\frac{1}{M}-1\right)
$$

we have

$$
\frac{\epsilon}{\zeta}=\frac{1}{2}\left(\frac{v^{4}}{u^{4}} \frac{1}{M}-1\right) ; \text { that is, } \frac{1}{2} \frac{v^{4}}{u^{4}}\left(\frac{1}{M}-\frac{u^{4}}{v^{4}}\right)=\frac{1}{2}\left(\frac{v^{4}}{u^{4}} \frac{1}{M}-1\right)
$$

which is right.

So in the equation $\frac{\gamma}{\alpha}=\frac{1}{8} \frac{\Pi}{\Delta}$, if for a moment $(\Pi),(\Delta)$ are what $\Pi, \Delta$ become, the 
equation is $\frac{\delta}{\zeta}=\frac{1}{8} \frac{(\Pi)}{(\Delta)}$, that is, $\frac{1}{u^{8}} \frac{\Pi^{\prime}}{\Delta}=\frac{(\Pi)}{(\Delta)}$, or $(\Pi)=\frac{1}{u^{8}} \frac{(\Delta)}{\Delta} \Pi^{\prime} ;$ but obviously $\frac{(\Delta)}{\Delta}=-\frac{1}{u^{4} v^{4}}$; and the equation thus is $(\Pi)=-\frac{1}{u^{12} v^{4}} \Pi^{\prime}$, or say $u^{12} v^{4}(\Pi)=-\Pi^{\prime}$; that is,

$$
\begin{aligned}
-\Pi^{\prime}=u^{12} v^{4} & \left\{\frac{v^{12}}{u^{12}} \cdot \frac{1}{M^{3}} \cdot \frac{1}{v^{4}}\left(1-\frac{1}{v^{8}}\right)\right. \\
+ & \frac{v^{8}}{u^{8}} \cdot \frac{1}{M^{2}} \cdot \frac{1}{u^{3}}\left(\frac{1}{u}-\frac{2}{v^{3}}\right)\left(1-\frac{1}{v^{8}}\right) \\
+ & \frac{v^{4}}{u^{4}} \cdot \frac{1}{M} \cdot \frac{4}{u^{7} v^{7}}+\frac{1}{v^{4}}\left(1-\frac{3}{u^{8}}\right)-\frac{4}{u^{3} v^{3}}+\frac{2}{u^{4}} \\
& \left.-\frac{2}{v^{4}}+\frac{6}{v^{3} u^{3}}\left(1-\frac{1}{u^{8}}\right)+\frac{1}{u^{4}}\left(-3+\frac{5}{u^{8}}\right)\right\},
\end{aligned}
$$

which is right.

The general theory by q-transcendents. Art. Nos. 52 to 71 .

52. I recur to the formula

$$
\frac{1-y}{1+y}=\frac{1-x}{1+x}\left(\frac{\alpha-\beta x+\gamma x^{2}+. . \pm \sigma n^{\frac{1}{2}(n-1)}}{\alpha+\beta x+\gamma x^{2}+\ldots+\sigma n^{\frac{1}{2}(n-1)}}\right)^{2},
$$

and seek to express the ratios $\alpha: \beta: \ldots: \sigma$ in terms of $q$. Writing with Jacobi

we have in general

$$
\omega=\frac{m K+m^{\prime} i K^{\prime}}{n}
$$

$$
\begin{gathered}
\alpha+\beta x+\gamma x^{2}+\ldots+\sigma x^{\frac{1}{(n-1)}}=\alpha\left(1+\frac{x}{\operatorname{snc} 2 \omega}\right)\left(1+\frac{x}{\operatorname{snc} 4 \omega}\right) \ldots\left(1+\frac{x}{\operatorname{snc}(n-1) \omega}\right) \\
(\operatorname{snc}=\sin \text { co } a m ; \text { viz. snc } 2 \omega=\operatorname{sn}(K-2 \omega), \text { \&c. })
\end{gathered}
$$

and the values of $\alpha, \beta, \ldots, \theta$ which correspond to the moduli $v_{0}, v_{1}, \ldots v_{n}$, or say the values $\left(\alpha_{0}, \beta_{0}, \ldots, \theta_{0}\right),\left(\alpha_{1}, \beta_{1}, \ldots, \theta_{1}\right), \ldots,\left(\alpha_{n}, \beta_{n}, \ldots, \theta_{n}\right)$, are obtained by giving to $\omega$ the values

$$
\begin{array}{cccc}
\omega_{0}, & \omega_{1}, & \omega_{2} & , \ldots, \omega_{n}, \\
= & \frac{2 K}{n}, \frac{2 K+i K^{\prime}}{n}, & \frac{4 K+i K^{\prime}}{n}, \ldots, \frac{i K^{\prime}}{n},
\end{array}
$$

viz. the cases $\omega_{0}, \omega_{n}$ correspond to Jacobi's first and second real transformations, and the others to the imaginary transformations.

I remark that $\omega=\omega_{0}$ gives for snc $2 g \omega$ an expression which is rational as regards $q$, but $\omega=\omega_{n}$ gives an expression involving $q^{\frac{1}{n}}$, the real $n$th root of $q$; the other values $\omega_{1}, \omega_{2}, \ldots$ give the like expressions, involving $\alpha q^{\frac{1}{n}}, \alpha^{2} q^{\frac{1}{n}}, \ldots(\alpha$ an imaginary $n$th root of unity), the imaginary $n$th roots of $q$.

$$
20-2
$$


156

A MEMOIR ON THE TRANSFORMATION OF ELLIPTIC FUNCTIONS.

$[578$

53. I consider first the expression

$$
\frac{1}{\operatorname{snc} 2 g \omega_{0}},=\frac{1}{\operatorname{sn}\left(K-2 g \omega_{0}\right)}, \quad=\frac{\operatorname{dn} 2 g \omega_{0}}{\operatorname{cn} 2 g \omega_{0}} .
$$

Here, writing $2 g \omega_{0}=\frac{2 K \xi}{\pi}$ ( $\xi$ for Jacobi's $x$, as $x$ is being used in a different sense), that is,

$$
\xi=\frac{\pi}{2 K} \cdot 2 g \cdot \frac{2 K}{n},=\frac{2 g \pi}{n},
$$

(and thence $e^{i \xi}=e^{g \frac{2 \pi i}{n}}=\alpha^{g}, e^{2 i \xi}=\alpha^{2 g}$, if $\alpha=e^{\frac{2 \pi i}{n}}$, an imaginary $n$th root of unity), we have (Jacobi, p. 86, [Gee. Werke, t. I., p. 143])

$$
\begin{aligned}
\frac{1}{\operatorname{snc} 2 g \omega_{0}} & =\operatorname{dn} \frac{2 K \xi}{\pi} \div \operatorname{cn} \frac{2 K \xi}{\pi} \\
& =\frac{C}{B} \cdot \frac{2 e^{i \xi}}{1+e^{2 i \xi}} \cdot \frac{\left(1+q e^{2 i \xi}\right) \ldots\left(1+q e^{-2 i \xi}\right) \ldots}{\left(1+q^{2} e^{2 i \xi}\right) \ldots\left(1+q^{2} e^{-2 i \xi}\right) \ldots}
\end{aligned}
$$

where

$$
\bar{B}=\left\{\frac{\left(1+q^{2}\right) . . .}{(1+q)}\right\}^{2}=f^{2}(q)
$$

that is,

$$
\frac{1}{\operatorname{snc} 2 g \omega_{0}}=\frac{2 \alpha^{g}}{1+\alpha^{2 g}} \cdot f^{2}(q) \cdot \frac{\left(1+\alpha^{2 g} q\right) \ldots\left(1+\alpha^{n-2 g} q\right) \ldots}{\left(1+\alpha^{2 g} q^{2}\right) \ldots\left(1+\alpha^{n-2 g} q\right) \ldots},
$$

where, for shortness, I write $\left(1+q e^{2 i \xi}\right) \ldots$ to denote the infinite product

$$
\left(1+q e^{2 i \xi}\right)\left(1+q^{3} e^{2 i \xi}\right)\left(1+q^{5} e^{2 i \xi}\right) \ldots
$$

and similarly $\left(1+q^{2} e^{2 i \xi}\right) \ldots$ to denote the infinite product $\left(1+q^{2} e^{2 i \xi}\right)\left(1+q^{4} e^{2 i \xi}\right)\left(1+q^{6} e^{2 i \xi}\right) \ldots$, and the like for the terms in $e^{-2 i \xi}$ : the notation, accompanied by its explanation, is quite intelligible, and it would be difficult to make one which would be at the same time complete and not cumbrous. Then attributing to $g$ the values $1,2, \ldots, \frac{1}{2}(n-1)$, and forming the symmetric functions of these expressions, we have the values of $\frac{\beta}{\alpha}, \frac{\gamma}{\alpha}, \& \mathrm{c}$., or $\alpha$ being put $=1$, say the values of $\beta, \gamma, \ldots, \sigma$.

54. I stop to notice a verification afforded by the value of $\beta_{0}$. Putting $u=0$, that is, $q=0$, we have

and thence

$$
\frac{1}{\operatorname{snc} 2 g \omega_{0}}=\frac{2 \alpha^{g}}{1+\alpha^{2 g}}
$$

$$
\beta_{0}=2\left\{\frac{\alpha}{1+\alpha^{2}}+\frac{\alpha^{2}}{1+\alpha^{4}}+\frac{\alpha^{3}}{1+\alpha^{6}}+\ldots+\frac{\alpha^{\frac{1}{3}(n-1)}}{1+\alpha^{n-1}}\right\}
$$
we have $2 \beta_{0}=\frac{1}{M_{0}}-1$; and putting as above $u=0$, the value of $\frac{1}{M_{0}}$ is $=(-)^{\frac{n-1}{2}} n$;
whence

$$
(-)^{\frac{1}{2}(n-1)} n-1=4\left\{\frac{\alpha}{1+\alpha^{2}}+\frac{\alpha^{2}}{1+\alpha^{4}}+\frac{\alpha^{3}}{1+\alpha^{6}}+\ldots+\frac{\alpha^{\frac{1}{2}(n-1)}}{1+\alpha^{n-1}}\right\},
$$

www.rcin.org.pl 
a theorem relating to the imaginary $n$th roots of unity, $n$ an odd prime. In particular,

$$
\begin{aligned}
& n=3, \quad-4=4\left\{\frac{\alpha}{1+\alpha^{2}}\right\}, \text { at once verified by } \alpha^{2}+\alpha+1=0 ; \\
& n=5, \quad 4=4\left\{\frac{\alpha}{1+\alpha^{2}}+\frac{\alpha^{2}}{1+\alpha^{4}}\right\}, \text { verified by } \alpha^{5}-1=0,
\end{aligned}
$$

viz. the theorem is also true for the real root $\alpha=1$; in fact, the term in \{\} is

$$
\begin{gathered}
\left\{\alpha\left(1+\alpha^{4}\right)+\alpha^{2}\left(1+\alpha^{2}\right)\right\} \div\left(1+\alpha^{2}\right)\left(1+\alpha^{4}\right), \text { that is, }\left(\alpha+1+\alpha^{2}+\alpha^{4}\right) \div\left(1+\alpha^{2}+\alpha^{4}+\alpha\right),=1 ; \\
n=7, \quad-8=4\left\{\frac{\alpha}{1+\alpha^{2}}+\frac{\alpha^{2}}{1+\alpha^{4}}+\frac{\alpha^{3}}{1+\alpha^{6}}\right\}
\end{gathered}
$$

which may be verified by means of $\alpha^{6}+\alpha^{5}+\alpha^{4}+\alpha^{3}+\alpha^{2}+\alpha+1=0$; and so on.

55. I further remark that we have

$$
\frac{1}{M_{0}}=(-)^{\frac{1}{2}(n-1)}\left\{\frac{\operatorname{sn} 2 \omega_{0} \cdot \operatorname{sn} 4 \omega_{0} \ldots \operatorname{sn}(n-1) \omega_{0}}{\operatorname{snc} 2 \omega_{0} \cdot \operatorname{snc} 4 \omega_{0} \ldots \operatorname{snc}(n-1) \omega_{0}}\right\}^{2} .
$$

But Jacobi (p. 86, [l.c.]),

$$
\begin{aligned}
\operatorname{sn} 2 g \omega_{0} & =\operatorname{sn} \frac{2 K \xi}{\pi} \\
& =\frac{A K}{\pi i} \frac{e^{2 i \xi}-1}{e^{i \xi}} \frac{\left(1-q^{2} e^{2 i \xi}\right) \ldots\left(1-q^{2} e^{-2 i \xi}\right) \ldots}{\left(1-q e^{2 i \xi}\right) \ldots\left(1-q e^{-2 i \xi}\right) . .}
\end{aligned}
$$

where (p. 89, [l.c., p. 146])

that is,

$$
\frac{A K}{\pi}=\frac{\sqrt[4]{q}}{\sqrt{k}}, \quad=\frac{1}{2}\left\{\frac{(1+q)\left(1+q^{3}\right) \cdots}{\left(1+q^{2}\right)\left(1+q^{4}\right) \ldots}\right\}^{2}=\frac{1}{2} f^{-2}(q)
$$

Hence

$$
\operatorname{sn} 2 g \omega=f^{-2} q \cdot \frac{\alpha^{2 g}-1}{2 i \alpha^{g}} \frac{\left(1-\alpha^{2 g} q^{2}\right) \ldots\left(1-\alpha^{n-2 g} q^{2}\right) \ldots}{\left(1-\alpha^{2 g} q\right) \ldots\left(1-\alpha^{n-2 g} q\right) \ldots} \text {. }
$$

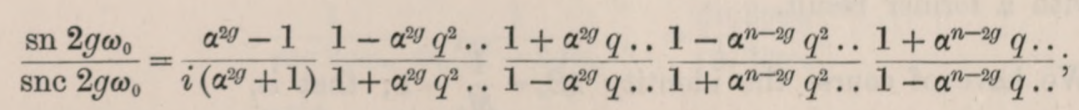

and giving to $g$ the values $1,2, \ldots, \frac{1}{2}(n-1)$, and multiplying the several expressions, we have the value of $\frac{1}{M_{0}}$, viz. this is

$$
\frac{1}{M_{0}}=(-)^{\frac{1}{2}(n-1)} \Pi\left\{\frac{\left(\alpha^{2 g}+1\right)^{2}}{i^{2}\left(\alpha^{2 g}+1\right)^{2}}\right\} R(q),
$$

where $R(q)$ denotes the product of the several factors which contain $q$.

56. The $\left(i^{2}\right)$ of the denominator gives a factor $i^{n-1},=(-)^{\frac{n-1}{2}}$, which destroys the factor $(-)^{\frac{n-1}{2}}$. We have then a factor

$$
\Pi\left(\frac{\alpha^{2 g}-1}{\alpha^{2 g}+1}\right)^{2}, \text { which is }=(-)^{\frac{1}{2}(n-1)} n .
$$


In fact, $n=3$, this is

$$
\left(\frac{\alpha^{2}-1}{\alpha^{2}+1}\right)^{2}=-3
$$

viz. the numerator is $\alpha-2 \alpha^{2}+1,=-3 \alpha^{2}$, and the denominator is $(-\alpha)^{2},=\alpha^{2}$.

So $n=5$, the formula is

$$
\left(\frac{\alpha^{2}-1}{\alpha^{2}+1} \cdot \frac{\alpha^{4}-1}{\alpha^{4}+1}\right)^{2}=5, \text { that is, } \frac{\left(\alpha^{2}-1\right)^{4}}{\left(\alpha^{4}+1\right)^{2}}=5
$$

or

$$
\frac{\alpha^{3}-4 \alpha^{2}+6 \alpha^{4}-4 \alpha+1}{\alpha^{3}+2 \alpha^{4}+1}=5,
$$

viz. this is $5\left(1+\alpha^{3}+2 \alpha^{4}\right)-\left(1-4 \alpha-4 \alpha^{2}+\alpha^{3}+6 \alpha^{4}\right)=0$, which is right; and so in other cases.

We thus have

$$
\frac{1}{M_{0}}=(-)^{\frac{1}{2}(n-1)} n \cdot R(q)
$$

which, on putting therein $u=0$, that is, $q=0$, gives, as it should do, $\frac{1}{M_{0}}=(-)^{\frac{1}{2}(n-1)} n$.

57. As regards the expression of $R(q)$, observe that, giving to $g$ its different values, the factors $1-a^{2 g} q^{2}$ and $1-a^{n-2 g} q^{2}$ are all the factors other than $1-q^{2}$ of $1-q^{2 n}$, and so as to the other pairs of factors; viz. we have

viz. this is

$$
R(q)=\left(\frac{1-q^{2 n} . .}{1-q^{2} . .} \frac{1+q^{n} \cdot .}{1+q \cdots} \frac{1+q^{2} \cdot \cdots}{1+q^{2 n} \cdot .} \frac{1-q \cdots}{1-q^{n} . .}\right)^{2},
$$

that is,

$$
=\left(\frac{1-q^{2 n} \cdot .}{1+q^{2 n} \cdot .} \frac{1+q^{n} \cdot \cdot}{1-q^{n} \cdot .}\right)^{2} \div\left(\frac{1-q^{2} \cdot .1+q \cdot \cdot}{1+q^{2} \cdot \cdot}\right)^{2}
$$

agreeing with a former result.

$$
\frac{1}{M_{0}}=(-)^{\frac{1}{2}(n-1)} n \frac{\phi^{2}\left(q^{n}\right)}{\phi^{2}(q)}
$$

58. We have of course the identity $2 \beta_{0}=\frac{1}{M_{0}}-1$; that is,

$$
4 S \frac{a^{g}}{1+\alpha^{2 g}} f^{2}(q) \cdot \frac{\left(1+\alpha^{2 g} q\right) \ldots\left(1+\alpha^{n-2 g} q\right) \ldots}{\left(1+\alpha^{2 g} q^{2}\right) \ldots\left(1+\alpha^{n-2 g} q^{2}\right) . .}=(-)^{\frac{1}{2}(n-1)} n \frac{\phi^{2}\left(q^{n}\right)}{\phi^{2}(q)}-1,
$$

$\left(g=1,2, \ldots \frac{1}{2}(n-1)\right)$, which, putting therein $q=0$, is an identity before referred to; a form perhaps more convenient is obtained by dividing each side by $f^{2}(q)$.

59. I notice further that we have

the term in \{\} is

$$
v_{0}=u^{n}\left\{\operatorname{snc} 2 \omega_{0} \operatorname{snc} 4 \omega_{0} \ldots \operatorname{snc}(n-1) \omega_{0}\right\} ;
$$

$$
\Pi \frac{1+\alpha^{2 g}}{2 \alpha^{g}} f^{-g}(q) \frac{\left(1+\alpha^{2 g} q^{2}\right) \ldots\left(1+\alpha^{n-2 g} q\right)^{2} \ldots}{\left(1+\alpha^{g} q\right) \ldots\left(1+\alpha^{n-2 g} q\right) \ldots},
$$

where we have $\Pi \frac{1+\alpha^{2 g}}{\alpha}=(-)^{\frac{2}{8}\left(n^{2}-1\right)}$. For example, $n=3$, the term is $\frac{1+\alpha^{2}}{\alpha}=-1$; 
$n=5$, it is

$n=7$, it is

$$
\frac{\left(1+\alpha^{2}\right)\left(1+\alpha^{4}\right)}{\alpha \cdot \alpha^{2}},=\frac{1+\alpha^{2}+\alpha^{4}+\alpha}{\alpha^{3}},=-1 ;
$$

$$
\frac{\left(1+\alpha^{2}\right)\left(1+\alpha^{4}\right)\left(1+\alpha^{6}\right)}{\alpha \cdot \alpha^{2} \cdot \alpha^{4}},=\frac{1+\alpha+\alpha^{2}+\alpha^{3}+\alpha^{4}+\alpha^{5}+2 \alpha^{6}}{\alpha^{6}}=1 ;
$$

and so on. The term in question thus is

that is,

$$
(-)^{\frac{2}{3}\left(n^{2}-1\right)} \cdot \frac{1}{(\sqrt{2})^{n-1}} f^{-n+1}(q) \frac{1+q^{2 n} \cdot .1+q \cdots}{1+q^{n} \cdots 1+q^{2} \cdots},
$$

$$
(-)^{\frac{2}{3}\left(n^{2}-1\right)} \frac{1}{(\sqrt{2})^{n-1}} f^{-n}(q) f\left(q^{n}\right) \text {. }
$$

This has to be multiplied by $u^{n},=(\sqrt{2})^{n} q^{\frac{n}{8}} f^{n}(q)$, and we thus obtain

agreeing with a former result.

$$
v_{0}=(-)^{\frac{1}{8}\left(n^{2}-1\right)} \sqrt{2} q^{\frac{n}{8}} f\left(q^{n}\right)
$$

We have in what precedes a complete $q$-transcendental solution for the transformatio prima; viz. the original modulus $k^{2}\left(=u^{8}\right)$ being given as a function of $q$, then, as well the new modulus $\lambda_{0}{ }^{2}\left(=v_{0}{ }^{8}\right)$ and the multiplier $M_{0}$, as also the several functions which enter into the expression

$$
\frac{1-y}{1+y}=\frac{1-x}{1+x}\left\{\frac{\left(1-\frac{x}{\operatorname{snc} 2 \omega_{0}}\right) \ldots\left(1-\frac{x}{\operatorname{snc}(n-1) \omega_{0}}\right)}{\left(1+\frac{x}{\operatorname{snc} 2 \omega_{0}}\right) \ldots\left(1+\frac{x}{\operatorname{snc}(n-1) \omega_{0}}\right)}\right\}^{2},
$$

are all of them expressed as functions of $q$.

60. I consider in like manner the expression

$$
\frac{1}{\operatorname{snc} 2 g \omega_{n}} \frac{1}{\operatorname{sn}\left(K-2 g \omega_{n}\right)}, \quad=\frac{\operatorname{dn} 2 g \omega_{n}}{\operatorname{cn} 2 g \omega_{n}} .
$$

Here, writing $2 g \omega_{n}=\frac{2 K \xi}{\pi}$ ( $\xi$ instead of Jacobi's $x$ as before), that is,

and thence

$$
\xi=\frac{\pi}{2 K} \cdot 2 g \cdot \frac{i K^{\prime}}{n}=\frac{g \pi i K^{\prime}}{n K}
$$

we have

$$
e^{i \xi}=e^{-\frac{g}{n} \frac{\pi K^{\prime}}{K}},=q^{\frac{g}{n}}
$$

$$
\begin{aligned}
\frac{1}{\text { snc } 2 g \omega_{n}} & =\operatorname{dn} \frac{2 K \xi}{\pi} \div \operatorname{cn} \frac{2 K \xi}{\pi} \\
& =f^{2}(q) \cdot \frac{2 q^{\frac{g}{n}}}{1+q^{\frac{2 g}{n}}} \cdot \frac{\left(1+q^{1+\frac{2 g}{n}}\right) \ldots\left(1+q^{1-\frac{2 g}{n}}\right) \ldots}{\left(1+q^{2+\frac{2 g}{n}}\right) \ldots\left(1+q^{2-\frac{2 g}{n}}\right) \ldots}
\end{aligned}
$$


where the notations are as follows:

and

$$
\left(1+q^{1+\frac{2 g}{n}}\right) \ldots \text { is the infinite product }\left(1+q^{1+\frac{2 g}{n}}\right)\left(1+q^{3+\frac{2 g}{n}}\right)\left(1+q^{5+\frac{2 g}{n}}\right) \ldots
$$

$$
\left(1+q^{2+\frac{2 g}{n}}\right) \ldots \text { is the infinite product }\left(1+q^{2+\frac{2 g}{n}}\right)\left(1+q^{4+\frac{2 g}{n}}\right)\left(1+q^{6+\frac{2 g}{n}}\right) \ldots
$$

and the like as to the expressions with exponents containing $-\frac{2 g}{n}$.

And then attributing to $g$ the values $1,2, \ldots, \frac{1}{2}(n-1)$, and forming the symmetric functions of these expressions, we have the values of $\frac{\beta}{\alpha}, \frac{\gamma}{\alpha}, \ldots, \frac{\sigma}{\alpha}$; or $\alpha$ being put $=1$, say the values of $\beta, \gamma, \ldots, \sigma$.

It is easy to see, and I do not stop to prove that, if instead of $\omega=\omega_{n}$ we have $\omega=\omega_{1}, \omega_{2}, \ldots$, or $\omega_{n-1}$, we simply multiply $q^{\frac{1}{n}}$ by an imaginary $n$th root of unity; that is, we replace the real $n$th root $q^{\frac{1}{n}}$ by an imaginary $n$th root of $q$.

In the case $u=0$, that is, $q=0$, we have $\frac{1}{\operatorname{snc} 2 g \omega_{n}}=0$, and thence $\beta=0$; and the like for the values $\omega_{1}, \omega_{2}, \ldots, \omega_{n-1}$ : the equation $2 \beta=\frac{1}{M}-1$ gives consequently for $\frac{1}{M}, n$ values each $=1$, agreeing with the multiplier equation.

61. We have for $M_{n}$ the formula

and, as before,

$$
\frac{1}{M_{n}}=(-)^{\frac{1}{2}(n-1)}\left\{\frac{\operatorname{sn} 2 \omega_{n} \operatorname{sn} 4 \omega_{n} \ldots \operatorname{sn}(n-1) \omega_{n}}{\operatorname{snc} 2 \omega_{n} \operatorname{snc} 4 \omega_{n} \ldots \operatorname{snc}(n-1) \omega_{n}}\right\}^{2},
$$

$$
\operatorname{sn} 2 g \omega_{n}=f^{-2}(q) \cdot \frac{q^{\frac{2 g}{n}}-1}{2 i q^{\frac{g}{n}}} \frac{\left(1-q^{2+\frac{2 g}{n}}\right) \ldots}{\left(1-q^{1+\frac{2 g}{n}}\right) \ldots\left(1-q^{1-\frac{2 g}{n}}\right) \ldots}
$$

hence

$$
\frac{\operatorname{sn} 2 g \omega_{n}}{\operatorname{snc} 2 g \omega_{n}}=\frac{q^{\frac{2 g}{n}}-1}{i\left(q^{\frac{2 g}{n}}+1\right)} \cdot \frac{\left(1-q^{2+\frac{2 g}{n}}\right) \ldots}{\left(1+q^{2+\frac{2 g}{n}}\right) \ldots\left(1-q^{1+\frac{2 g}{n}}\right) \ldots\left(1+q^{2-\frac{2 g}{n}}\right) \ldots\left(1-q^{1-\frac{2 g}{n}}\right) \ldots}
$$

and we thence derive the value of $\frac{1}{M_{n}}$; viz. observing that we have in the denominator $\left(i^{2}\right)^{\frac{1}{2}(n-1)},=(-)^{\frac{1}{2}(n-1)}$ which destroys this factor in the expression of $\frac{1}{M_{n}}$, this is

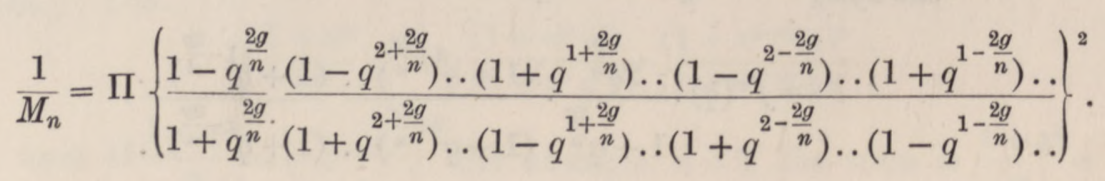


Now, giving to $g$ its values, it is easy to see that we have

$$
\Pi\left(1-q^{\frac{2 g}{n}}\right)\left(1-q^{2+\frac{2 g}{n}}\right) \ldots\left(1-q^{2-\frac{2 g}{n}}\right) . .=\frac{\left(1-q^{\frac{2}{n}}\right) . .}{\left(1-q^{2}\right) \ldots},
$$

where $\left(1-q^{\frac{2}{n}}\right)$. denotes $\left(1-q^{\frac{2}{n}}\right)\left(1-q^{\frac{4}{n}}\right)\left(1-q^{\frac{6}{n}}\right) \ldots$, viz. it is the same function of $q^{\frac{1}{n}}$ that $\left(1-q^{2}\right)$. is of $q$; also

$$
\Pi\left(1+q^{1+\frac{2 g}{n}}\right) . .\left(1+q^{1-\frac{2 g}{n}}\right) . .=\frac{\left(1+q^{\frac{1}{n}}\right) \ldots}{(1+q) \ldots},
$$

where $\left(1+q^{\frac{1}{n}}\right)$.. denotes $\left(1+q^{\frac{1}{n}}\right)\left(1+q^{\frac{3}{n}}\right)\left(1+q^{\frac{5}{n}}\right) \ldots$ viz. it is the same function of $q^{\frac{1}{n}}$ that $(1+q) .$. is of $q$; and the like as to the denominator factors: we thus have

viz. this is

$$
\frac{1}{M_{n}}=\left\{\frac{\left(1-q^{\frac{2}{n}}\right) . .}{\left(1-q^{2}\right) \ldots\left(1+q^{\frac{1}{n}}\right) . .} \frac{\left(1+q^{2}\right) \ldots}{(1+q) \ldots\left(1+q^{\frac{2}{n}}\right) \ldots\left(1-q^{\frac{1}{n}}\right) . .}\right\}^{2},
$$

or, we have

$$
=\left\{\frac{\left(1-q^{\frac{2}{n}}\right) . .}{\left(1+q^{\frac{2}{n}}\right) . .} \frac{\left(1-q^{\frac{1}{n}}\right) . .}{\left(1+q^{\frac{1}{n}}\right.}\right\}^{2} \div\left\{\frac{\left(1-q^{2}\right) . .(1+q) . .}{\left(1+q^{2}\right) . .(1-q) . .}\right\}^{2}
$$

agreeing with a former result.

$$
\frac{1}{M_{n}}=\phi^{2}\left(q^{\frac{1}{n}}\right) \div \phi^{2}(q)
$$

We have

$$
2 \beta_{n}=\frac{1}{M_{n}}-1
$$

that is,

$$
2\left\{\frac{1}{\operatorname{snc} 2 \omega_{n}}+\frac{1}{\operatorname{snc} 4 \omega_{n}} \ldots+\frac{1}{\operatorname{snc}(n-1) \omega_{n}}\right\}=\frac{\phi^{2}\left(q^{\frac{1}{n}}\right)}{\phi^{2}(q)}-1,
$$

a result which, substituting on the left-hand side the foregoing values of the several functions, must be identically true.

62. We have also

$$
v_{n}=u^{n}\left\{\operatorname{snc} 2 \omega_{n} \operatorname{snc} 4 \omega_{n} \ldots \operatorname{snc}(n-1) \omega_{n}\right\}
$$

where the term in \{\} is

$$
=\Pi f^{-2}(q) \frac{\left(1+q^{\frac{2 g}{n}}\right)}{2 q^{\frac{g}{n}}} \frac{\left(1+q^{2+\frac{2 g}{n}}\right) . .\left(1+q^{2-\frac{2 g}{n}}\right) .}{\left(1+q^{1+\frac{2 g}{n}}\right) . .\left(1+q^{1-\frac{2 g}{n}}\right) . .}
$$

or, observing that the sum of the exponents $\frac{g}{n}$ is $\frac{1}{n}\left\{1+2 . .+\frac{1}{2}(n-1)\right\}=\frac{n^{2}-1}{8 n}$, this is

$$
=f^{-n+1}(q) \cdot \frac{1}{(\sqrt{2})^{n-1} q^{\frac{n^{2}-1}{8 n}}} \frac{\left(1+q^{\frac{2}{n}}\right) \ldots(1+q) \ldots}{\left(1+q^{2}\right) \ldots\left(1+q^{\frac{1}{n}}\right) \ldots}
$$

C. IX. 
or, the last factor being $f\left(q^{\frac{1}{n}}\right) \div f(q)$, the expression is

$$
f^{-n}(q) \frac{1}{(\sqrt{2})^{n-1}} q^{-\frac{n}{8}+\frac{1}{8 n}} f\left(q^{\frac{1}{n}}\right)
$$

or, multiplying by $u^{n},=(\sqrt{2})^{n} q^{\frac{n}{8}} f^{n}(q)$, we have

agreeing with a former result.

$$
v_{n}=\sqrt{2} q^{\frac{1}{8 n}} f\left(q^{\frac{1}{n}}\right)
$$

We have in what precedes the complete $q$-transcendental solution for the transformatio secunda; viz. the original modulus $k\left(=u^{4}\right)$ being given as a function of $q$, then, as well the new modulus $\lambda_{n}\left(=v_{n}{ }^{4}\right)$ and the multiplier $M_{n}$, as also the several functions which enter into the formula

$$
\frac{1-y}{1+y}=\frac{1-x}{1+x}\left\{\frac{\left(1-\frac{x}{\operatorname{snc} 2 \omega_{n}}\right) \ldots\left(1-\frac{x}{\operatorname{snc}(n-1) \omega_{n}}\right)}{\left(1+\frac{x}{\operatorname{snc} 2 \omega_{n}}\right) \ldots\left(1+\frac{x}{\operatorname{snc}(n-1) \omega_{n}}\right)}\right\}^{2}
$$

are all expressed in terms of $q$. The expressions all contain $q^{\frac{1}{n}}$, and by substituting for this an imaginary $n$th root of $q$, we have the formulæ belonging to the several $(n-1)$ imaginary transformations.

63. As an illustration of the formulæ for the transformatio secunda I write $n=7$; and putting for greater convenience $q=r^{7}$, that is, $r=q^{\frac{1}{7}}$, then we have

where

$$
\begin{gathered}
v_{7}=\sqrt{2} r^{\frac{1}{8}} f(r), \quad \frac{1}{M_{7}}=\frac{\phi^{2}(r)}{\phi^{2}\left(r^{7}\right)}, \\
\frac{1}{\operatorname{snc} 2 \omega_{7}}=2 f^{2}\left(r^{7}\right) A, \quad \frac{1}{\operatorname{snc} 4 \omega_{7}}=2 f^{2}\left(r^{7}\right) B, \quad \frac{1}{\operatorname{snc} 6 \omega_{7}}=2 f^{2}\left(r^{7}\right) C,
\end{gathered}
$$

$$
\begin{aligned}
& A=r \cdot \frac{5.19 \ldots 9.23 \ldots}{2.16 \ldots 12.26 \ldots} \\
& B=r^{2} \cdot \frac{3.17 \ldots 11.25 \ldots}{4.18 \ldots 10.24 \ldots} \\
& C=r^{3} \cdot \frac{1.15 \ldots 13.27 \ldots}{6.20 \ldots 8.22 \ldots}
\end{aligned}
$$

where the numerator of $A$ denotes $\left(1+r^{5}\right)\left(1+r^{19}\right) \ldots\left(1+r^{9}\right)\left(1+r^{29}\right) \ldots$, and so in other cases, the difference of the exponents being always $=14$. And we have, as mentioned, the identical equation

$$
f^{2}\left(r^{7}\right)(A+B+C)=\frac{1}{4}\left\{\frac{\phi^{2} r}{\phi^{2} r^{7}}-1\right\} .
$$

The values of the several expressions up to $r^{50}$ are as follows: Mr J. W. L. Glaisher kindly performed for me the greater part of the calculation. 


\begin{tabular}{|c|c|c|c|c|c|c|}
\hline $\begin{array}{l}\text { Ind. } \\
\text { of } r\end{array}$ & $A$ & $B$ & $C$ & Sum & $\begin{array}{l}\text { Multiplied by } \\
\qquad f^{2}\left(r^{7}\right)\end{array}$ & $\begin{array}{c}\phi^{2}(r) \\
\div \phi^{2}\left(r^{7}\right)\end{array}$ \\
\hline 0 & & & & 0 & 0 & + \\
\hline 1 & +1 & & & +1 & + & + \\
\hline 2 & & +1 & & & & + \\
\hline 3 & -1 & & +1 & 0 & 0 & 0 \\
\hline 4 & & & +1 & & & + \\
\hline 5 & +1 & +1 & & +2 & $\begin{array}{l}2 \\
+\quad 2\end{array}$ & + \\
\hline 6 & +1 & -1 & & 0 & 0 & 0 \\
\hline 7 & -1 & & & -1 & -1 & $-\quad 4$ \\
\hline 8 & -1 & & & -1 & $-\quad 3$ & $-\quad 12$ \\
\hline 9 & +1 & -1 & -1 & -1 & -3 & $\begin{array}{l}-\quad 12 \\
-1\end{array}$ \\
\hline 10 & +2 & +1 & -1 & +2 & +2 & + \\
\hline 11 & -1 & & -1 & -2 & -4 & $-\quad 16$ \\
\hline 12 & -2 & -1 & -1 & -4 & -8 & $\begin{array}{l}-\quad 32 \\
\end{array}$ \\
\hline 13 & & $\begin{array}{r}1 \\
+\quad 2\end{array}$ & & +2 & $\begin{array}{r}2 \\
+\quad 2\end{array}$ & $\begin{array}{r}8 \\
+\quad 8\end{array}$ \\
\hline 14 & +2 & -1 & & +1 & +3 & $+\quad 12$ \\
\hline 15 & +1 & -1 & +1 & +1 & $\begin{array}{r}8 \\
+\quad 8\end{array}$ & $\begin{array}{r}32 \\
+\quad 32\end{array}$ \\
\hline 16 & -2 & +2 & +2 & +2 & $\begin{array}{r}9 \\
+\quad 9\end{array}$ & $\begin{array}{l}+\quad 36\end{array}$ \\
\hline 17 & -2 & -2 & +2 & -2 & -6 & $-\quad 24$ \\
\hline 18 & +1 & +1 & +2 & +4 & $+\quad 13$ & $\begin{array}{l}+52 \\
+\end{array}$ \\
\hline 19 & +2 & +2 & +2 & +6 & +24 & $\begin{array}{l}+96 \\
\end{array}$ \\
\hline 20 & & -3 & +1 & -2 & -6 & $-\quad 24$ \\
\hline 21 & -2 & +2 & -1 & -1 & -8 & - $\quad 32$ \\
\hline 22 & -2 & +1 & -2 & -3 & $-\quad 20$ & $-\quad 80$ \\
\hline 23 & +2 & -4 & -3 & -5 & -24 & $-\quad 96$ \\
\hline 24 & +3 & +3 & -4 & +2 & +16 & $+\quad 64$ \\
\hline 25 & & -1 & -4 & -5 & $-\quad 33$ & -132 \\
\hline 26 & -4 & -3 & -3 & -10 & -62 & -248 \\
\hline 27 & -2 & +5 & -1 & +2 & +16 & $+\quad 64$ \\
\hline 28 & +4 & -3 & +1 & +2 & $\begin{array}{r}19 \\
+\quad 19\end{array}$ & +76 \\
\hline 29 & +5 & -1 & +3 & +7 & $\begin{array}{r}46 \\
+\quad 46\end{array}$ & +184 \\
\hline 30 & -3 & +6 & +5 & +8 & $\begin{array}{r}+56 \\
\end{array}$ & +224 \\
\hline 31 & -7 & -6 & +7 & -6 & -40 & -160 \\
\hline 32 & +1 & +1 & +7 & +9 & $\begin{array}{l}+77 \\
\end{array}$ & +308 \\
\hline 33 & +9 & +5 & +4 & +18 & +144 & +576 \\
\hline 34 & +3 & -8 & +1 & -4 & $\begin{array}{l}1 \\
-\quad 38\end{array}$ & -152 \\
\hline 35 & -9 & +5 & -1 & -5 & -42 & -168 \\
\hline 36 & -7 & $\begin{array}{r}+2 \\
+\quad\end{array}$ & -5 & -10 & $\begin{array}{r}-\quad 99 \\
-\end{array}$ & $-\quad 396$ \\
\hline 37 & +7 & -9 & -9 & -11 & -122 & -488 \\
\hline 38 & +11 & +10 & -11 & +10 & $\begin{array}{r}1 \\
+\quad 88\end{array}$ & $\begin{array}{r}152 \\
+\quad 352\end{array}$ \\
\hline 39 & -4 & -3 & -10 & -17 & -168 & -672 \\
\hline 40 & -13 & -8 & -7 & -28 & -310 & -1240 \\
\hline 41 & -2 & +13 & -3 & +8 & +82 & $\begin{array}{r}+328 \\
\end{array}$ \\
\hline 42 & +13 & -8 & +3 & +8 & +88 & $+\quad 352$ \\
\hline 43 & +8 & -3 & +9 & +14 & +204 & +816 \\
\hline 44 & -11 & +14 & +14 & +17 & +252 & +1008 \\
\hline 45 & -14 & -14 & +16 & -12 & -182 & -728 \\
\hline 46 & +5 & +4 & +15 & +24 & +344 & +1376 \\
\hline 47 & +17 & +11 & +12 & +40 & +632 & +2528 \\
\hline 48 & +3 & -20 & +5 & -12 & -168 & -672 \\
\hline 49 & -17 & +13 & -5 & -9 & -175 & -700 \\
\hline 50 & -13 & +5 & -14 & -22 & -401 & -1604 \\
\hline
\end{tabular}


64. As already mentioned, the foregoing expressions of the coefficients in terms of $q$ may be applied to the determination of the coefficients as rational functions of $u$, $v$.

Representing by $\theta$ any one of the coefficients $\alpha, \beta, \gamma, \ldots, \sigma$, consider the sum

$$
S \frac{v^{f} \theta}{\alpha}
$$

$f$ a positive integer, and the summation extending as before to the $n+1$ values of $v$, and corresponding values of $\frac{\theta}{\alpha}$. This is a rational function of $u$, and it is also integral. As to this observe that the function, if not integral, must become infinite either for $u=0$ (this would mean that the expression contained a term or terms $\left.A u^{-a}\right)$ or for some finite value of $u$. But the function can only become infinite by reason of some term or terms of $S v^{f} \frac{\theta}{\alpha}$ becoming infinite; viz. some term $\frac{1}{\operatorname{snc} 2 g \omega}$ must become infinite; or attending to the equation

$$
v=u^{n}\{\operatorname{snc} 2 \omega \operatorname{snc} 4 \omega \ldots \operatorname{snc}(n-1) \omega\},
$$

it can only happen if $u=0$, or if $v=\infty$; and from the modular equation it appears that if $v=\infty$, then also $u=\infty$ : the expression in question can therefore only become infinite if $u=0$, or if $u=\infty$. Now $u=0$ gives the ratios $\frac{\beta}{\alpha}, \frac{\gamma}{\alpha}, \ldots$, each of them a determinate function of $n$, that is finite; and gives also $v=0$, so that the expression does not become infinite for $u=0$; hence it does not become infinite either for $u=0$ or for any finite value of $u$; wherefore it is integral. The like reasoning applies to the sum $S v^{-f} \frac{\theta}{\alpha}$; viz. this is a rational function of $u$; and it is quasi-integral, viz. there are no terms having a denominator other than a power of $u$, the highest denominator being $u^{n f}$; viz. the expression contains negative and positive integer powers of $u$, the lowest power (highest negative power) being $\frac{1}{u^{n f}}$.

65. It is to be observed, further, that writing the expression in the form

$$
v_{0}^{f} \frac{\theta_{0}}{\alpha_{0}}+S^{\prime} v^{f} \frac{\theta}{\alpha}
$$

(where $S^{\prime}$ refers to the values $v_{1}, v_{2}, \ldots, v_{n}$ of the modulus), and considering the several quantities as expressed in terms of $q$, then in the sum $S^{\prime \prime}$ every term involving a fractional power $q^{\frac{h}{n}}$ acquires by the summation the coefficient $\left(1+\alpha+\alpha^{2}+\ldots+\alpha^{n-1}\right)$, and therefore disappears; there remains only the radicality $q^{\frac{1}{3}}$ occurring in the expressions of the $v$ 's; and if $n f \equiv \mu(\bmod .8), \mu=0$, or a positive integer less than 8 , then the form of the expression is $q^{\frac{\mu}{8}}$ into a rational function of $q$. Hence this, being a rational and integral function of $u$, must be of the form

$$
A u^{\mu}+B u^{\mu+8}+C u^{\mu+16}+\& c \text {. }
$$


66. We have thus in general

and in like manner

$$
S v^{f} \frac{\theta}{\alpha}=A w^{\mu} \quad+B u^{\mu+8} \quad+\& c .
$$

$$
S v^{-f} \frac{\theta}{\alpha}=A^{\prime} u^{-n f}+B^{\prime} u^{-n f+8}+\& c .
$$

We may in these expressions find a limit to the number of terms, by means of the before-mentioned theorem that we may simultaneously interchange $u, v ; \alpha, \beta, \ldots, \rho, \sigma$ into $\frac{1}{u}, \frac{1}{v} ; \sigma, \rho, \ldots, \beta, \alpha$. Starting from the expression of $S v^{f} \frac{\theta}{\alpha}$, let $\phi$ be the corresponding coefficient to $\theta$; viz. in the series $\alpha, \beta, \ldots, \theta, \ldots, \phi, \ldots, \rho, \sigma$, let $\phi$ be as removed from $\sigma$ as $\theta$ is from $\alpha$; then the equation becomes

$$
S v^{-f} \frac{\phi}{\sigma}=A u^{-\mu}+B u^{-\mu-8}+\& c .
$$

where $\frac{\phi}{\sigma}=\frac{\phi}{\alpha} \frac{\alpha}{\sigma}=\frac{v}{u^{n}} \frac{\phi}{\alpha} ;$ the equation thus is

$$
S v^{1-f} \frac{\phi}{\alpha}=A u^{n-\mu}+B u^{n-\mu-8}+\& c .
$$

and by what precedes the series on the right-hand side can contain no negative power higher than $\frac{1}{u^{n(f-1)}}$; that is, the series of coefficients $A, B, C, \ldots$ goes on to a certain point only, the subsequent coefficients all of them vanishing.

In like manner from the equation for $S v^{-f}{ }_{\alpha}$ we have

$$
S v^{f+1} \frac{\phi}{\alpha}=A^{\prime} u^{(n+1) f}+B^{\prime} u^{(n+1) f-8}+\& \mathrm{c} .
$$

where the indices must be positive; viz. the series of coefficients $A^{\prime}, B^{\prime}, \ldots$ goes on to a certain point only, the subsequent coefficients all of them vanishing.

67. The like theory applies to the expression $\frac{1}{M}$. We have, putting as before $n f \equiv \mu(\bmod .8)$,

$$
\begin{aligned}
& S v^{f} \frac{1}{M}=A w^{\mu}+B w^{\mu+8}+\ldots, \\
& S v^{-f} \frac{1}{M}=A^{\prime} u^{-n f}+B^{\prime} u^{-n f+8}+\ldots
\end{aligned}
$$

and we find a limit to the number of terms by the consideration that we may simultaneously change $u, v, \frac{1}{M}$ into $\frac{1}{u}, \frac{1}{v}, \frac{v^{4}}{u^{4} M}$; the equations thus become

$$
S v^{4-1} \frac{1}{M}=A u^{4-\mu}+B u^{-4-\mu}+\ldots
$$


(where, if $f=$ or $<4$, there must be on the right-hand side no negative power of $u$; but if $f>4$, then the highest negative power must be $\left.\frac{1}{u^{(f-4) n}}\right)$, and

$$
S v^{4+f} \frac{1}{M}=A^{\prime} u^{n f+4}+B^{\prime} u^{n f-4}+\ldots
$$

where on the right-hand side there must be no negative power of $u$.

68. It is to be remarked that $\beta, \rho$ being always given linearly in terms of $\frac{1}{M}$ it is the same thing whether we seek in this manner for the values of $\beta, \rho$ or for that of $\frac{1}{M}$; but the latter course is practically more convenient. Thus in the cases $n=5, n=7$ we require only the value of $\frac{1}{M}$.

In the case $n=11$, where the coefficients are $\alpha, \beta, \gamma, \delta, \epsilon, \zeta$, it has been seen that $\gamma, \delta$ are given as cubic functions of $\frac{1}{M}$ : seeking for them directly, their values would (if the process be practicable) be obtained in a better form, viz. instead of the denominator $\left(F^{\prime} v\right)^{3}$ there would be only the denominator $F^{\prime \prime}(v)$.

69. I consider for $\frac{1}{M}$ the cases $n=3$ and 5 :

$$
n=3, f=0,1,2,3, \text { then } \mu=0,3,6,1 ;
$$

and we write down the equations

$$
\begin{array}{ll}
S \frac{1}{M}=A, & \text { giving } S \frac{v^{4}}{M}=A u^{4}, \\
S \frac{v}{M}=A^{\prime} u^{3}, & , \quad S \frac{v^{3}}{M}=A^{\prime} u, \\
S \frac{v^{2}}{M}=0, & , \quad S \frac{v^{2}}{M}=0 ;
\end{array}
$$

viz. if we had in the first instance assumed $S \frac{1}{M}=A+B u^{8}+\ldots$, this would have given $S \frac{v^{4}}{M}=A u^{4}+B u^{-4}+\ldots$, whence $B$ and the succeeding coefficients all vanish; and so in other cases. We have here only the coefficients $A, A^{\prime}$; and these can be obtained without the aid of the $q$-formulæ by the consideration that for $u=1$ the corresponding values of $v, \frac{1}{M}$ are

$$
\begin{aligned}
& v=1,-1,-1,-1 \\
& \frac{1}{M}=3,-1,-1,-1
\end{aligned}
$$


whence $A=0, A^{\prime}=6$; or we have the equations

giving as before

$$
S \frac{1}{M}=0, \quad S \frac{v}{M}=6 u^{3}, \quad S \frac{v^{2}}{M}=0, \quad S \frac{v^{3}}{M}=6 u,
$$

$$
\left(2 v^{3}+3 v^{2} u-u\right) \frac{1}{M}=3\left(v^{2} u^{2}+2 u^{5} v+1\right) u,
$$

reducible by means of the modular equation to $\frac{1}{M}=1+\frac{2 u^{3}}{v}$.

70. $n=5$. Corresponding to $f=0,1,2,3,4,5$, we have $\mu=0,5,2,7,4,1$, and we find

$$
\begin{array}{lll}
S \frac{1}{M}=A, & \text { giving } S \frac{v^{4}}{M}=A u^{4}, \\
S \frac{v}{M}=0, & , & S \frac{v^{3}}{M}=0, \\
S \frac{v^{2}}{M}=A^{\prime} u^{2}, & & S \frac{v^{2}}{M}=A^{\prime} u^{2}, \\
S \frac{v^{5}}{M}=A^{\prime \prime} u+B^{\prime \prime} u^{9}, & & S v^{-1} \frac{1}{M}=A^{\prime \prime} u^{3}+B^{\prime \prime} u^{-5} .
\end{array}
$$

But for $u=1$ the corresponding values of $v, \frac{1}{M}$ are

$$
\begin{aligned}
& v=1,-1,-1,-1,-1,-1, \\
& \frac{1}{M}=5, \quad 1, \quad 1, \quad 1, \quad 1, \quad 1 \text {; }
\end{aligned}
$$

whence $A=A^{\prime}=10, A^{\prime \prime}+B^{\prime \prime}=0$, or say the value of $S \frac{v^{5}}{M}$ is $=A^{\prime \prime} u\left(1-u^{8}\right)$.

The value of $A^{\prime \prime}$ is found very easily by the $q$-formulæ, viz. neglecting higher powers of $q$, we have

hence

$$
u=q^{\frac{1}{8}} \sqrt{2}, v_{0}=q^{\frac{5}{8}} \sqrt{2}, \frac{1}{M_{0}}=5 ; v_{5}=q^{\frac{1}{40}} \sqrt{2}, \frac{1}{M_{5}}=1 ;
$$

$$
S \frac{v^{5}}{M}=\frac{v_{0}^{5}}{M}+S^{\prime} \frac{v^{5}}{M},=5 q^{\frac{1}{8}}(\sqrt{2})^{5}=A^{\prime \prime} q^{\frac{1}{8}} \sqrt{2}
$$

that is, $A^{\prime \prime}=20$, and the equations are

$$
S \frac{1}{M}=10, \quad S \frac{v}{M}=0, \quad S \frac{v^{2}}{M}=10 u^{2}, \quad S \frac{v^{3}}{M}=0, \quad S \frac{v^{4}}{M}=10 u^{4}, \quad S \frac{v^{5}}{M}=20 u\left(1-u^{8}\right)
$$

whence

$$
\begin{aligned}
F^{\prime} v \cdot \frac{1}{M}= & 20 u\left(1-u^{8}\right) \\
& -10 u^{4}\left(S v_{0}-v\right) \\
& -10 u^{2}\left(S v_{0} v_{1} v_{2}-v S v_{0} v_{1}+v^{2} S v_{0}-v^{3}\right) \\
& -10\left(S v_{0} v_{1} v_{2} v_{3} v_{4}-v S v_{0} v_{1} v_{2} v_{3}+v^{2} S v_{0} v_{1} v_{2}-v^{3} S v_{0} v_{1}+v^{4} S v_{0}-v^{5}\right)
\end{aligned}
$$


where $S v_{0}$, \&c. are the coefficients of the equation

viz.

$$
v^{6}+4 v^{5} u^{5}+5 v^{4} u^{2}-5 v^{2} u^{4}-4 v u-u^{6}=0
$$

$S v_{0}, \quad v_{0} v_{1}, \quad v_{0} v_{1} v_{2}, \quad v_{0} v_{1} v_{2} v_{3}, \quad v_{0} v_{1} v_{2} v_{3} v_{4}$

are

or the equation is

$$
-4 u^{5},+5 u^{2}, \quad 0,-5 u^{4}, \quad 4 u
$$

$$
\begin{aligned}
F^{\prime} v \cdot \frac{1}{M}= & 20 u\left(1-u^{8}\right) \\
& -10 u^{4}\left(-4 u^{5}-v\right) \\
& -10 u^{2}\left(-5 u^{2} v-4 u^{5} v^{2}-v^{3}\right) \\
& -10\left(4 u+5 u^{4} v \quad-5 v^{3} u^{2}-4 v^{4} u^{5}-v^{5}\right)
\end{aligned}
$$

or, say

$$
\frac{1}{2} F^{\prime} v \frac{1}{M}=5\left\{v^{5}+4 v^{4} u^{5}+6 v^{3} u^{2}+4 v^{2} u^{7}+v u^{4}-2 u\left(1-u^{8}\right)\right\}
$$

where

$$
\frac{1}{2} F^{\prime} v \quad=3 v^{5}+10 v^{4} u^{5}+10 v^{3} u^{2} \quad-5 v u^{4}-2 u .
$$

Hence also, reducing by the modular equation,

$$
\frac{1}{2} v F^{\prime} v \frac{1}{M}=5 u\left\{v^{4} u+4 v^{3} u^{4}+6 v^{2} u^{3}+2 v\left(1+u^{8}\right)+u^{5}\right\},
$$

the one of which forms is as convenient as the other.

71. Making the change $u, v, \frac{1}{M}$ into $v,-u,-5 M$, we have

$$
-\frac{1}{2} F^{\prime} u .5 M=5\left\{-u^{5}+4 v^{5} u^{4}-6 v^{2} u^{3}+4 v^{7} u^{2}-v^{4} u-2 v\left(1-v^{8}\right)\right\}
$$

and comparing with the equation

$$
5 M^{2}=-\frac{\left(1-v^{8}\right) v F^{\prime} v}{\left(1-u^{8}\right) u F^{\prime} u}
$$

we obtain

$$
\frac{v\left(1-v^{8}\right)}{u\left(1-u^{8}\right)}=\frac{-2 v\left(1-v^{8}\right)-v^{4} u+4 v^{7} u^{2}-6 v^{2} u^{3}+4 v^{5} u^{4}-u^{5}}{-2 u\left(1-u^{8}\right)+u^{4} v+4 u^{7} v^{2}+6 u^{2} v^{3}+4 u^{5} v^{4}+v^{5}} .
$$

Writing for a moment $M=u^{4}+6 u^{2} v^{2}+v^{4}, N=u^{2}+v^{2}$, this is

that is,

$$
-\frac{v\left(1-v^{8}\right)}{u\left(1-u^{8}\right)}=\frac{-2 v\left(1-v^{8}\right)-u M+4 v^{5} u^{2} N}{-2 u\left(1-u^{8}\right)+v M+4 v^{2} u^{5} N}
$$

$$
-4 u v\left(1-u^{8}\right)\left(1-v^{8}\right)-\left\{u^{2}\left(1-u^{8}\right)-v^{2}\left(1-v^{8}\right)\right\} M+4 v^{3} u^{3}\left\{u^{2}\left(1-v^{8}\right)+v^{2}\left(1-u^{8}\right)\right\} N=0 .
$$

But we have

$$
\begin{aligned}
& u^{2}\left(1-u^{8}\right)-v^{2}\left(1-v^{8}\right)=\left(u^{2}-v^{2}\right)\left\{1-u^{8}-u^{6} v^{2}-u^{4} v^{4}-u^{2} v^{6}-v^{8}\right\} \\
& u^{2}\left(1-v^{8}\right)+v^{2}\left(1-u^{8}\right)=\left(u^{2}+v^{2}\right)\left\{1-u^{2} v^{2}\left(u^{4}-u^{2} v^{2}+v^{4}\right)\right\} .
\end{aligned}
$$


Hence, replacing $M, N$ by their values, this is

$$
\begin{aligned}
& -4 u v\left(1-u^{8}\right)\left(1-v^{8}\right) \\
& -\left(u^{2}-v^{2}\right)\left(1-u^{8}-u^{6} v^{2}-u^{4} v^{4}-u^{2} v^{6}-v^{8}\right)\left(u^{4}+6 u^{2} v^{2}+v^{4}\right) \\
& +4 u^{3} v^{3}\left(u^{2}+v^{2}\right)^{2}\left\{1-u^{2} v^{2}\left(u^{4}-u^{2} v^{2}+v^{4}\right)\right\}=0
\end{aligned}
$$

viz. writing $u^{2}-v^{2}=A$, $u v=B$, this is

$$
\begin{aligned}
& -4 B\left\{1-A^{4}-4 A^{2} B^{2}-2 B^{4}+B^{8}\right\} \\
& -\quad A\left\{1-A^{4}-5 A^{2} B^{2}-3 B^{4}\right\}\left(A^{2}+8 B^{2}\right) \\
& +4 B^{3}\left(A^{2}+4 B^{2}\right)\left\{1-A^{2} B^{2}-B^{4}\right\}=0,
\end{aligned}
$$

that is,

$$
\begin{aligned}
& -4 B\left\{\left(1-A^{4}-4 A^{2} B^{2}-2 B^{4}+B^{8}\right)-B^{2}\left(A^{2}+4 B^{2}\right)\left(1-A^{2} B^{2}-B^{4}\right)\right\} \\
& -A\left(1-A^{4}-5 A^{2} B^{2}-3 B^{4}\right)\left(A^{2}+8 B^{2}\right)=0
\end{aligned}
$$

viz.

$$
\begin{aligned}
& -4 B\left(1-A^{4}-5 A^{2} B^{2}-3 B^{4}\right)\left(1-B^{4}\right) \\
& -A\left(1-A^{4}-5 A^{2} B^{2}-3 B^{4}\right)\left(A^{2}+8 B^{2}\right)=0 ;
\end{aligned}
$$

or throwing out the factor $-\left(1-A^{4}-5 A^{2} B^{2}-3 B^{4}\right)$, this is

$$
A\left(A^{2}+8 B^{2}\right)+4 B\left(1-B^{4}\right)=0,
$$

the modular equation, which is right.

The four forms of the modular equation, and the curves represented thereby.

Art. Nos. 72 to 79 .

72. The modular equation for any value of $n$ has the property that it may be represented as an equation of the same order $(=n+1$, when $n$ is prime) between $u, v$ : or between $u^{2}, v^{2}$ : or between $u^{4}, v^{4}$ : or between $u^{8}, v^{8}$. As to this, remark that in general an equation $(u, v, 1)^{m}=0$ of the order $m$ gives rise to an equation $\left(u^{2}, v^{2}, 1\right)^{2 m}=0$ of the order $2 m$ between $u^{2}, v^{2}$; viz. the required equation is

$$
(u, v, 1)^{m}(u,-v, 1)^{m}(-u, v, 1)^{m}(-u,-v, 1)^{m}=0,
$$

where the left-hand side is a rational function of $u^{2}, v^{2}$ of the form $\left(u^{2}, v^{2}, 1\right)^{2 m}$; or again starting from a given equation $(u, v, w)^{m}=0$, and transforming by the equations $x: y: z=u^{2}: v^{2}: w^{2}$, the curve in $(x, y, z)$ is of the order $2 m$; in fact, the intersections of the curve by the arbitrary line $a x+b y+c z=0$ are given by the equations $(u, v, w)^{m}=0, a u^{2}+b v^{2}+c w^{2}=0$, and the number of them is thus $=2 m$. Moreover, by the general theory of rational transformation, the new curve of the order $2 m$ has the same deficiency as the original curve of the order $m$. The transformed curve in $x, y, z,=u^{2}, v^{2}, w^{2}$ may in particular cases reduce itself to a curve of the order $m$ twice repeated; but it is important to observe that here, taking the single curve of the order $m$ as the transformed curve, this has no longer the same deficiency as the original curve; and in particular the curves represented by the modular equation in its four several forms, writing therein successively $u, v ; u^{2}, v^{2} ; u^{4}, v^{4} ; u^{8}, v^{8},=x, y$, are not curves of the same deficiency.

73. The question may be looked at as follows: the quantities which enter rationally into the elliptic-function formulæ are $k^{2}, \lambda^{2}=u^{8}, v^{8}$; if a modular equation $(u, v)^{\nu}=0$ led to the transformed equation $\left(u^{8}, v^{8}\right)^{8 \nu}=0$, then to a given value of $u^{8}$ C. IX. 
would correspond 8 values of $u$, therefore $8 \nu$ values of $v$, giving the same number, $8 \nu$, values of $v^{8}$; that is, the values of $v^{8}$ corresponding to a given value of $u^{8}$ would group themselves in eights corresponding to the 8 values of $u$. There is, in fact, no such grouping; the equations are $(u, v)^{v}=0,\left(u^{8}, v^{8}\right)^{v}=0$; to a given value of $u^{8}$ correspond 8 values of $u$, and therefore $8 \nu$ values of $v$, but these give in eights the same value of $v^{8}$, so that the number of values of $v^{8}$ is $=\nu$.

74. I consider the case $n=3$ : here, writing $x, y$ for $u$, $v$, we have here the sextic curve

I. $y^{4}-x^{4}+2 x y\left(x^{2} y^{2}-1\right)=0$;

and it is easy to see that the remaining forms wherein $x, y$ denote $u^{2}, v^{2} ; u^{4}, v^{4}$; and $u^{8}, v^{8}$ respectively, are derived herefrom as follows; viz.

II. $\left(y^{2}-x^{2}\right)^{2}-4 x y(x y-1)^{2}=0$, that is,

$$
y^{4}+6 x^{2} y^{2}+x^{4}-4 x y\left(x^{2} y^{2}+1\right)=0
$$

III. $\left(y^{2}+6 x y+x^{2}\right)^{2}-16 x y(x y+1)^{2}=0$, that is,

$$
y^{4}+6 x^{2} y^{2}+x^{4}-4 x y\left(4 x^{2} y^{2}-3 x^{2}-3 y^{2}+4\right)=0
$$

IV. $\left(y^{2}+6 x y+x^{2}\right)^{2}-16 x y(4 x y-3 x-3 y+4)^{2}=0$, that is,

$$
y^{4}-762 x^{2} y^{2}+x^{4}-4 x y\left\{64 x^{2} y^{2}-96 x^{2} y-96 x y^{2}+33 x^{2}+33 y^{2}-96 x-96 y+64\right\}=0,
$$

where it may be noticed that the process is not again repeatable so as to obtain a sextic equation between $x, y$ standing for $u^{16}, v^{16}$ respectively.

The curve I. has a dp (fleflecnode) at the origin, viz. the branches are given by $y^{3}-2 x=0,-x^{3}-2 y=0$; and it has 2 cusps at infinity, on the axes $x=0, y=0$ respectively; viz. the infinite branches are given by $y+2 x^{3}=0,-x+2 y^{3}=0$ respectively. These same singularities present themselves in the other curves.

The curve II. has the four dps $\left(x^{2}-y^{2}=0, x y-1=0\right)$, that is,

$$
(x=y=1),(x=y=-1),(x=i, y=-i),(x=-i, y=i) \text {. }
$$

Corresponding hereto we have in the curve III. the 2 dps $(x=y=1, x=y=-1)$, and in the curve IV. the $\mathrm{d} p(x=y=1)$.

The curve III. has besides the 4 dps $y^{2}+6 x y+x^{2}=0, x y+1=0$, that is,

$$
(1+\sqrt{2}, 1-\sqrt{2}),(1-\sqrt{2}, 1+\sqrt{2}),(-1-\sqrt{2},-1+\sqrt{2}),(-1+\sqrt{2},-1-\sqrt{2}) ;
$$

and corresponding hereto in the curve IV. we have the $2 \mathrm{dps}$

$$
(3+2 \sqrt{2}, 3-2 \sqrt{2}),(3-2 \sqrt{2}, 3+2 \sqrt{2}) .
$$

The curve IV. has besides the 4 dps $\left(y^{2}+6 x y+x^{2}=0,4 x y-3 x-3 y+4=0\right)$, or say $\left(2 x-\frac{3}{2}\right)\left(2 y-\frac{3}{2}\right)+\frac{7}{4}=0,2\left(x+\frac{9}{4}\right)^{2}+2\left(y+\frac{9}{4}\right)^{2}-\frac{177}{8}=0$. Hence the 4 curves have respectively the dps and deficiency following:-

$$
\begin{array}{lrr}
\text { dps. } & \text { dps. } & \text { Def. } \\
2,1 & =3, & 7 \\
2,1,4 & =7, & 3 \\
2,1,2,4=9, & 1 \\
2,1,1,2,4=10, & 0
\end{array}
$$

viz. the curve IV. representing the equation between $u^{8}$ and $v^{8}$ is a unicursal sextic. 
It may be noticed that, except the fleflecnode at the origin and the cusps at infinity, the dps in question are all acnodes (conjugate points).

75. The foregoing equations may be exhibited in the square diagrams:-

I.

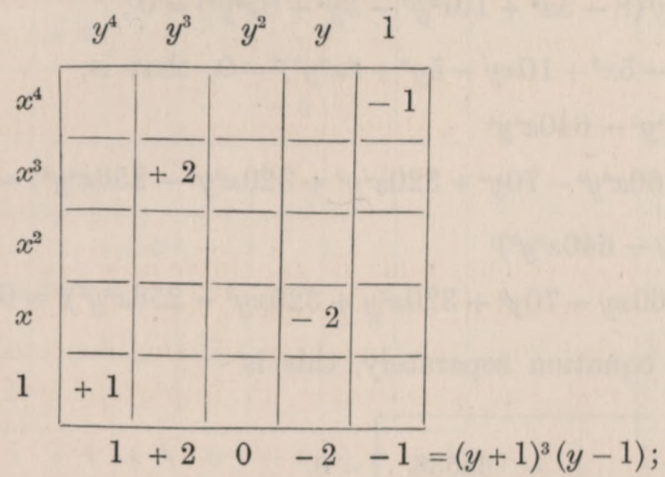

II.

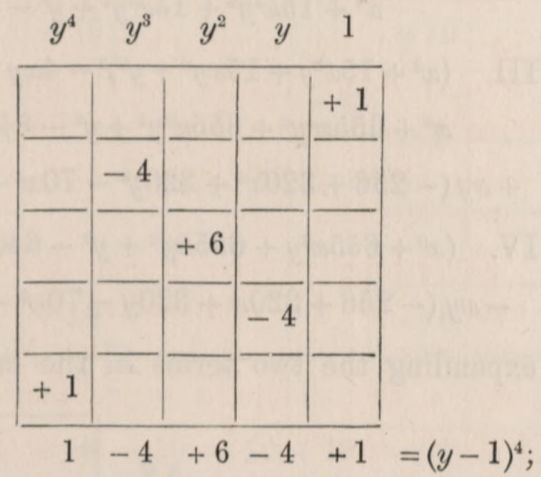

IV.

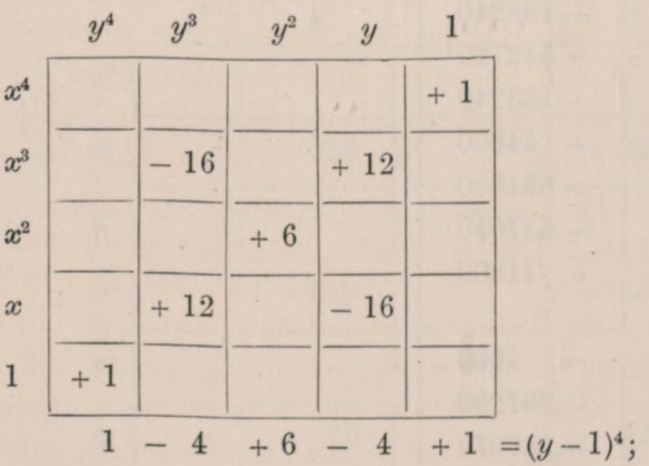

where the subscript line, showing in each case what the equation becomes on writing therein $x=1$, serves as a verification of the numerical values.

The curve IV. being unicursal, the coordinates may be expressed rationally in terms of a parameter; in fact, we have

These values give

$$
x=\frac{\alpha^{3}(2+\alpha)}{1+2 \alpha}, y=\frac{\alpha(2+\alpha)^{3}}{(1+2 \alpha)^{3}} .
$$

$$
\begin{array}{rlrl}
16 x y & =16 \alpha^{4}(2+\alpha)^{4} & \div(1+2 \alpha)^{4}, \\
4+4 x y-3 x-3 y & =(4,8,12,32,50,32,12,8,4 \gamma 1, \alpha)^{8} & \div(1+2 \alpha)^{4}, \\
x^{2}+6 x y+y^{2} & =4 \alpha^{2}(2+\alpha)^{3}(4,8,12,32,50,32,12,8,4 \gamma 1, \alpha)^{8} \div(1+2 \alpha)^{6},
\end{array}
$$

and the equation of the curve is thus verified. 
76. Considering in like manner the modular equation for the quintic transformation, we derive the four forms as follows:-

I. $x^{6} y^{6}+5 x^{2} y^{2}\left(x^{2}-y^{2}\right)+4 x y\left(1-x^{4} y^{4}\right)=0 ;$

II. $\left\{x^{3}-y^{3}+5 x y(x-y)\right\}^{2}-16 x y\left(1-x^{2} y^{2}\right)^{2}=0$, that is,

$$
x^{6}+15 x^{4} y^{2}+15 x^{2} y^{4}+y^{6}-2 x y\left(8-5 x^{4}+10 x^{2} y^{2}-5 y^{4}+8 x^{4} y^{4}\right)=0 ;
$$

III. $\left(x^{3}+15 x^{2} y+15 x y^{2}+y^{3}\right)^{2}-4 x y\left(8-5 x^{2}+10 x y-5 y^{2}+8 x^{2} y^{2}\right)^{2}=0$, that is, $x^{6}+655 x^{4} y^{2}+655 x^{2} y^{4}+y^{6}-640 x^{2} y^{2}-640 x^{4} y^{4}$

$+x y\left(-256+320 x^{2}+320 y^{2}-70 x^{4}-660 x^{2} y^{2}-70 y^{4}+320 x^{4} y^{2}+320 x^{2} y^{4}-256 x^{4} y^{4}\right)=0$;

IV. $\left(x^{3}+655 x^{2} y+655 x y^{2}+y^{3}-640 x y-640 x^{2} y^{2}\right)^{2}$

$-x y\left(-256+320 x+320 y-70 x^{2}-660 x y-70 u^{2}+320 x^{2} y+320 x y^{2}-256 x^{2} y^{2}\right)^{2}=0:$

or, expanding the two terms in the last equation separately, this is

\begin{tabular}{|c|c|c|}
\hline$x y$ & & -65536 \\
\hline$x^{2} y$ & & +163840 \\
\hline$x y^{2}$ & & +163840 \\
\hline$x^{3} y$ & & -138240 \\
\hline$x^{2} y^{2}$ & +409600 & -542720 \\
\hline$x y^{3}$ & & -138240 \\
\hline$x^{4} y$ & 1280 & +44800 \\
\hline$x^{3} y^{2}$ & -838400 & +631040 \\
\hline$x^{2} y^{3}$ & -838400 & +631040 \\
\hline$x y^{4}$ & 1280 & +44800 \\
\hline$x^{6}$ & + & \\
\hline$x^{5} y$ & 1310 & $-\quad 4900$ \\
\hline$x^{4} y^{2}$ & +430335 & -297200 \\
\hline$x^{3} y^{3}$ & +1677252 & -986072 \\
\hline$x^{2} y^{4}$ & +430335 & -297200 \\
\hline$x y^{5}$ & 1310 & $-\quad 4900$ \\
\hline$y^{6}$ & 1 & i \\
\hline$x^{5} y^{2}$ & - $\quad 1280$ & +44800 \\
\hline$x^{4} y^{3}$ & - 838400 & +631040 \\
\hline$x^{3} y^{4}$ & -838400 & +631040 \\
\hline$x^{2} y^{5}$ & $-\quad 1280$ & +44800 \\
\hline$x^{5} y^{3}$ & & -138240 \\
\hline$x^{4} y^{4}$ & +409600 & -542720 \\
\hline$x^{3} y^{5}$ & & -138240 \\
\hline$x^{5} y^{4}$ & & +163840 \\
\hline$x^{4} y^{5}$ & & +163840 \\
\hline$x^{5} y^{5}$ & & -65536 \\
\hline
\end{tabular}


77. The square diagrams are:-

I.

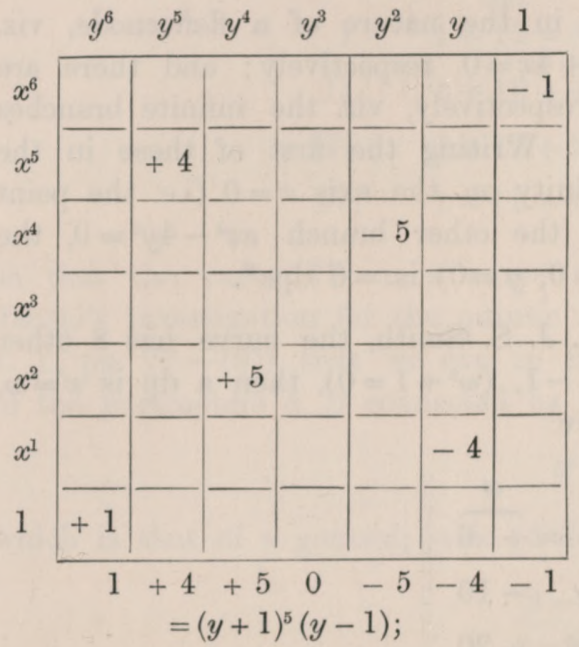

II.

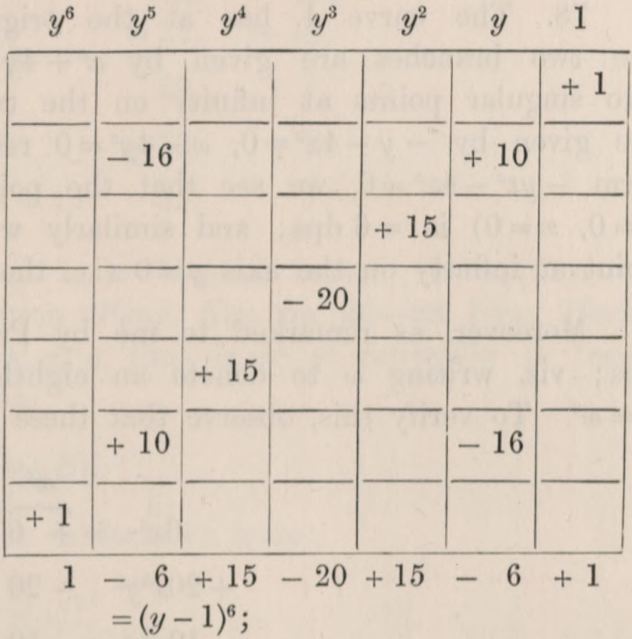

III.

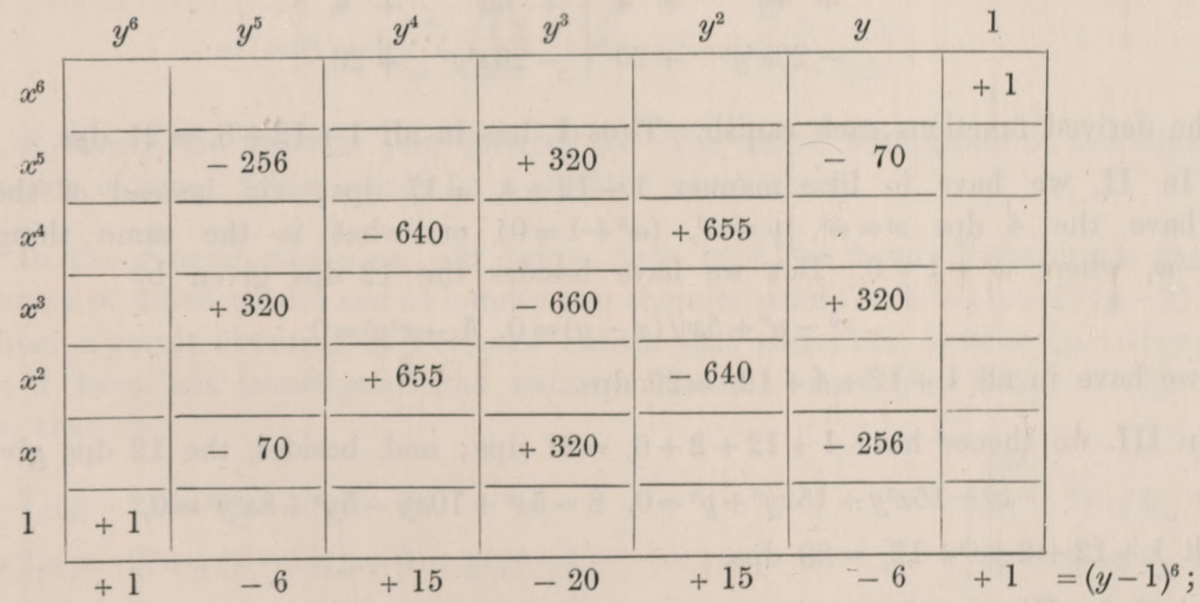

IV.

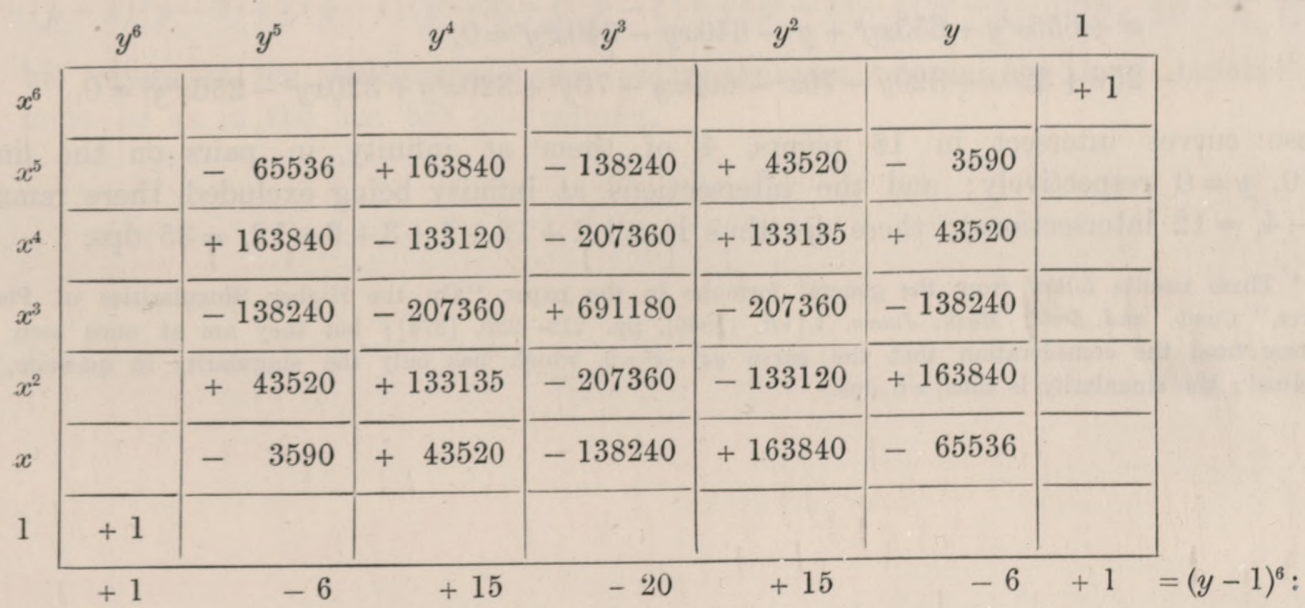


where the subscript line, showing in each case what the equation becomes on writing therein $x=1$, serves as a verification of the numerical values.

78. The curve I. has at the origin a $d p$ in the nature of a fleflecnode, viz. the two branches are given by $x^{5}+4 y=0,-y^{5}+4 x=0$ respectively; and there are two singular points at infinity on the two axes respectively, viz. the infinite branches are given by $-y-4 x^{5}=0, x-4 y^{5}=0$ respectively. Writing the first of these in the form $-y z^{4}-4 x^{5}=0$, we see that the point at infinity on the axis $x=0$ (i.e. the point $z=0, x=0)$ is $=6 \mathrm{dps}$; and similarly writing for the other branch $x z^{4}-4 y^{5}=0$, the point at infinity on the axis $y=0$ (i.e. the point $z=0, y=0$ ) is $=6 \mathrm{dps}$ *.

Moreover, as remarked to me by Professor H. J. S. Smith, the curve has 8 other dps; viz. writing $\omega$ to denote an eighth root of $-1,\left(\omega^{8}+1=0\right)$, then a dp is $x=\omega$, $y=\omega^{5}$. To verify this, observe that these values give

$$
\begin{array}{rr|ll}
6 x^{5} & =\overbrace{+6}^{\omega^{5}} & -6 y^{5} & =\overbrace{+6}^{\omega} \\
+20 x^{3} y^{2} & -20 & +10 x^{4} y & -10 \\
-10 x y^{4} & -10 & -20 x^{2} y^{3} & -20 \\
+4 y & +4 & +4 x & +4 \\
-20 x^{4} y^{5} & +20 & -20 x^{5} y^{4} & +20
\end{array}
$$

or the derived functions each vanish. Thus $I$. has in all $1+12+8,=21 \mathrm{dps}$.

In II. we have in like manner $1+12+4,=17 \mathrm{dps}$; viz. instead of the $8 \mathrm{dps}$, we have the $4 \mathrm{dps} x=\omega^{2}, y=\omega^{2},\left(\omega^{8}+1=0\right)$, or, what is the same thing, $x=\omega$, $y=-\omega$, where $\omega^{4}+1=0$. But we have besides the $12 \mathrm{dps}$ given by

$$
x^{3}-y^{3}+5 x y(x-y)=0,1-x^{2} y^{2}=0,
$$

viz. we have in all $1+12+4+12,=29 \mathrm{dps}$.

In III. we thence have $1+12+2+6,=21 \mathrm{dps}$; and, besides, the $12 \mathrm{dps}$ given by

$$
x^{3}+15 x^{2} y+15 x y^{2}+y^{3}=0,8-5 x^{2}+10 x y-5 y^{2}+8 x^{2} y^{2}=0,
$$

in all $1+12+2+6+12,=33$ dps.

And in IV. we thence have $1+12+1+3+6,=23 \mathrm{dps}$; and, besides, the $12 \mathrm{dps}$ given by

$$
\begin{aligned}
& x^{3}+655 x^{2} y+655 x y^{2}+y^{3}-640 x y-640 x^{2} y^{2}=0, \\
- & 256+320 x+320 y-70 x^{2}-660 x y-70 y^{2}+320 x^{2} y+320 x y^{2}-256 x^{2} y^{2}=0,
\end{aligned}
$$

(these curves intersect in 16 points, 4 of them at infinity, in pairs on the lines $x=0, y=0$ respectively; and the intersections at infinity being excluded, there remain $16-4,=12$ intersections); there are thus in all $1+12+1+3+6+12,=35 \mathrm{dps}$.

* These results follow from the general formulæ in the paper "On the Higher Singularities of Plane Curves," Camb. and Dubl. Math. Journ. t. vII. (1866), pp. 212-223, [374]; but they are at once seen to be true from the consideration that the curve $y z^{4}-x^{5}=0$, which has only the singularity in question, is unicursal; the singularity is thus $=6 \mathrm{dps}$. 
Arranging the results in a tabular form and adding the values of the deficiency, we have

$\begin{array}{rlrr}\text { I. } & 1+12+8 & \text { dps. } & \text { Def. } \\ \text { I. } & 21, & =15, \\ \text { II. } & 1+12+4+12 & 29, & 7, \\ \text { III. } & 1+12+2+6+12 & 33, & 3, \\ \text { IV. } & 1+12+1+3+6+12 & 35, & 1,\end{array}$

so that the curve IV. is a curve of deficiency 1, or bicursal curve. It appears by Jacobi's investigation for the quintic transformation (Fund. Nov. pp. 26-28, [Ges. Werke, t. I., pp. $77-79]$ ) that we can in fact express $x, y$, that is, $u^{8}, v^{8}$, rationally in terms of the parameters $\alpha, \beta$ connected by the equation

$$
\alpha^{3}=2 \beta(1+\alpha+\beta),
$$

which is that of a general cubic (deficiency $=1$ ); in fact, we have

that is,

$$
\frac{2-\alpha}{\alpha-2 \beta}=\frac{v^{4}}{u^{4}}, \quad \beta=\frac{u^{5}}{v},
$$

$$
u^{8}(=x)=\beta^{2}\left(\frac{2-\alpha}{\alpha-2 \beta}\right), \quad v^{8}(=y)=\beta^{2}\left(\frac{2-\alpha}{\alpha-2 \beta}\right)^{5},
$$

where $\alpha, \beta$ satisfy the relation just referred to. The actual verification of the equation IV. by means of these values would be a work of some labour.

79. In the general case $p$ an odd prime, then in I. we have at the origin one dp (in the nature of a fleflecnode) and at infinity two singular points each $=\frac{1}{2}(p-1)(p-2) d$ dps. I infer, from a result obtained by Professor Smith, that there are besides $(p-1)(p-3)$ dps; but I have not investigated the nature of these. And the Table of dps and deficiency then is

I. $1+(p-1)(p-2)+(p-1)(p-3)$

II. $1+(p-1)(p-2)+\frac{1}{2}(p-1)(p-3)+\frac{1}{2}\left(p^{2}-1\right)$

$=\mid \begin{array}{ll}\text { dps. } & \text { Def. } \\ 2 p^{2}-7 p+6, & 4 p-5, \\ 2 p^{2}-5 p+4, & 2 p-3, \\ 2 p^{2}-4 p+3, & p-2, \\ 2 p^{2}-\frac{7}{2} p+\frac{5}{2}, & \frac{1}{2} p-\frac{3}{2}\end{array}$

III. $1+(p-1)(p-2)+\frac{1}{4}(p-1)(p-3)+\frac{1}{4}\left(p^{2}-1\right)+\frac{1}{2}\left(p^{2}-1\right)$

IV. $1+(p-1)(p-2)+\frac{1}{8}(p-1)(p-3)+\frac{1}{8}\left(p^{2}-1\right)+\frac{1}{4}\left(p^{2}-1\right)+\frac{1}{2}\left(p^{2}-1\right)$

$p^{2}-\frac{7}{2} p+\frac{5}{2}, \frac{1}{2} p-\frac{3}{2}$

viz. his values of the deficiencies being as in the last column, the total number of dps must be as in the last but one column. 